\title{
Clone Detection in Matlab Stateflow Models
}

\author{
by \\ JIAN CHEN
}

\author{
A thesis submitted to the \\ School of Computing \\ in conformity with the requirements for \\ the degree of Master of Science
}

Queen's University

Kingston, Ontario, Canada

September 2014

Copyright $\odot$ Jian Chen, 2014 


\begin{abstract}
Matlab Simulink is one of the leading tools for model based software development in the automotive industry. One extension to Simulink is Stateflow, which allows the user to embed Statecharts as components in a Simulink Model. These state machines contain nested states, an action language that describes events, guards, conditions and actions and complex transitions. As Stateflow has become increasingly important in Simulink models for the automotive sector, we extend previous work on clone detection of Simulink models to Stateflow components.

In this thesis, we present an approach for identifying Stateflow clones in Matlab Stateflow models. In order to leverage robust near-miss code clone technology, our approach is text-based. First, we transform the Stateflow textual representation into a hierarchical textual structure. We implement a SIMONE plugin that normalizes the initial input to remove irrelevant elements and rename irrelevant naming differences to make the process of clone identification more accurate. Finally, we identify potential clone candidates and cluster them into classes.

We conducted experiments with our approach on the Matlab Simulink/Stateflow Demo set. Our approach showed promising results on the identification of Stateflow clones as an isolated component as well as an integrated component of the Simulink models that are hosting them. All of our results are manually validated.
\end{abstract}




\section{Statement of Co-Authorship}

The work presented in this thesis was accomplished under the supervision of Dr. Thomas R. Dean and Dr. Manar H. Alalfi, who provided feedback and corrections to the manuscript. Parts of this thesis, specifically parts of Chapter 2 and Chapter 3, have been published in the proceedings of the 8th International Workshop on Software Clones (IWSC 2014). 


\section{Acknowledgments}

This work is supported in part by NSERC(Natural Sciences and Engineering Research

Council), as part of the NECSIS Automotive Partnership, and by the Ontario Research Fund through a Research Excellence grant.

I would like to express my deepest gratitude towards my supervisors Dr. Thomas R. Dean and Dr. Manar H. Alalfi for their patience, thoughtful suggestions, and time. 


\section{Contents}

Abstract

Statement of Co-Authorship ii

Acknowledgments $\quad$ iii

Contents $\quad$ iv

List of Figures $\quad$ vi

List of Code Listings $\quad$ x

List of Tables $\quad$ xii

Chapter 1: Introduction 1

1.1 Motivation . . . . . . . . . . . . . . . . 1

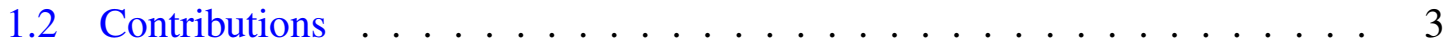

1.3 Thesis Outline . . . . . . . . . . . . . . . . . 4

Chapter 2: $\quad$ Background 5

2.1 Stateflow Models . . . . . . . . . . . . . . . . . . 5

2.2 Source Transformation . . . . . . . . . . . . . . . . 10

2.2.1 The TXL Source Transformation Language . . . . . . . . . . . . . . . 10

2.3 Clone Detection . . . . . . . . . . . . . . . . . 11

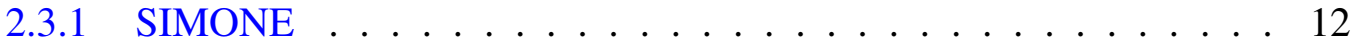

2.4 Related work . . . . . . . . . . . . . . . . . . . . . . . . . . . . . . . 13

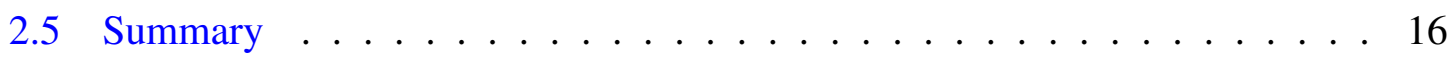

$\begin{array}{ll}\text { Chapter 3: } & 17\end{array}$

3.1 Clones in Stateflow Models . . . . . . . . . . . . . . . . . . 18

3.2 Clone Classification in Stateflow Models . . . . . . . . . . . . . . . 20

3.3 Stateflow TXL Grammar . . . . . . . . . . . . . . . 22 
3.4 Representation Transformation . . . . . . . . . . . . . . . 22

3.5 Extractor Plugin . . . . . . . . . . . . . . . . . . . . 26

3.6 Normalization . . . . . . . . . . . . . . . . 26

3.7 Contextualization . . . . . . . . . . . . . . 28

3.8 Evaluation . . . . . . . . . . . . . . . . . . 28

3.9 Summary . . . . . . . . . . . . . . . . . . . . 29

Chapter 4: $\quad$ Representation Transformation 30

4.1 MDL File Structure . . . . . . . . . . . . . . . . . . . 30

4.2 Structure Folding . . . . . . . . . . . . . . . . . . . . . 32

4.3 Label Splitting . . . . . . . . . . . . . . . . . . . . . . . . . . . . . . . . . . . . . . .

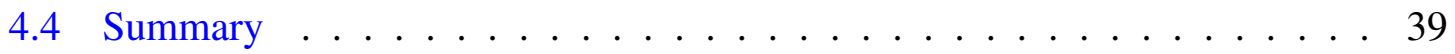

Chapter 5: $\quad$ Extraction And Normalization 40

5.1 Extraction . . . . . . . . . . . . . . . . 41

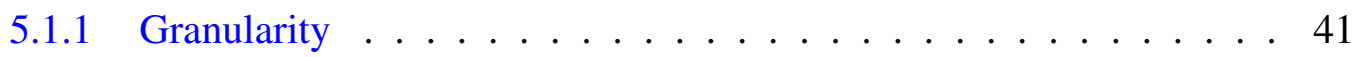

5.1 .2 Extractor Plugin . . . . . . . . . . . . . . . 41

5.1.3 Clone Detection Results . . . . . . . . . . . . . . 42

5.2 Normalization .......................... 43

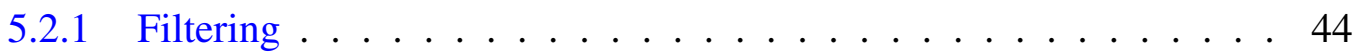

5.2 .2 Renaming . . . . . . . . . . . . . . . 48

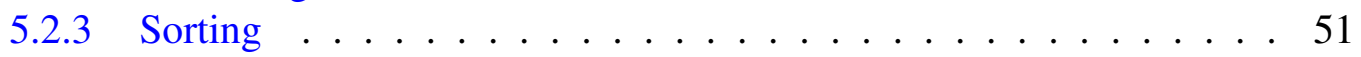

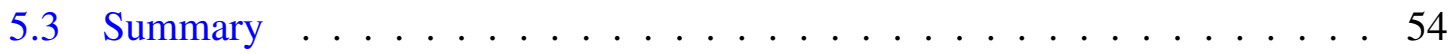

Chapter 6: Contextualization $\quad \mathbf{5 5}$

6.1 Simulink \& Stateflow Environment . . . . . . . . . . . . . . . 55

6.1 Graphic Representation . . . . . . . . . . . . . . . . . 56

6.1 .2 Textual Representation . . . . . . . . . . . . . . 57

6.2 States contextualization . . . . . . . . . . . . . . . . 59

6.2 .1 Contextualization via full lines . . . . . . . . . . . . . . 60

6.2 .2 Contextualization via one line . . . . . . . . . . . . . . 65

6.2.3 Contextualization via weighted lines . . . . . . . . . . . . . 69

6.2.4 Contextualization discussion . . . . . . . . . . . . . . 73

6.3 Summary ............................ 74

Chapter 7: $\quad$ Conclusions and Future Work 75

7.1 Summary . . . . . . . . . . . . . . . 75

7.2 Future Work . . . . . . . . . . . . . . . . 77 


\section{List of Figures}

2.1 A simple example of a Stateflow block within a Simulink model . . . . . . 6

2.2 A simple Stateflow example. The chart is the parent of the state A, state A is the parent of the $\mathrm{B}, \mathrm{C}$, and $\mathrm{D}$ states. Exclusive state $\mathrm{D} 1$ and $D 2$. Parallel state $C 1$ and $C 2$. The chart also includes a history junction and a Simulink function, and transitions junctions $\ldots \ldots \ldots 6$

2.3 Example snippet of the textual representation in MDL file . . . . . . . 8

2.4 Example snippet of the textual representation used by Stateflow . . . . . . . 9

3.1 Steps of our approach . . . . . . . . . . . . . . . 18

3.2 Steps of contextualization . . . . . . . . . . . . . . . . 19

3.3 Example of a clone in a Stateflow model. Both (a) and (b) in the sf_aircraft_ screen_library model and they contain the same number of states and transitions. The two failure_logging states can be considered as the cloned

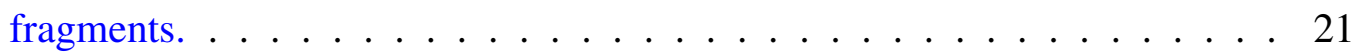

3.4 Example snippet of the textual representation used by Stateflow . . . . . . . 23

3.5 Simplified example of folded Stateflow model . . . . . . . . . . . . . . 24

3.6 Example of a split state label . . . . . . . . . . . . . 25

4.1 Representation Transformation: explicit model hierarchy and separate each action to its own attribute . . . . . . . . . . . . . 31 
4.2 Stateflow Hierarchy of Objects[19] . . . . . . . . . . . . . . . 32

4.3 Textual Presentation of powerwindow model chart control . . . . . . . . 33

4.5 Folding textual presentation result of powerwindow model . . . . . . . 36

5.1 Normalization transformation steps: extraction \& filtering, renaming, and sorting. ........................... 40

5.2 Example of Stateflow clones from sldemo_auto_climate_elec.mdl and sldemo_ auto_climatecontrol.mdl in Matlab demo automotive models . . . . . . . . 47

5.3 A Type 2(renamed model clone), this example is in the powerwindow model. The four red states are similar to each other. SIMONE similarity 76\% . . . 49

5.4 A Type 3(near-miss model clone), both function e and $r$ in the same chart Game in sf_tetris model. The function form the of fragment of clone. SIMONE similarity $71 \%$. . . . . . . . . . . . . . . . 49

5.5 A Type 3(near-miss model clone), (a) in sf_boiler model and (b) in sldemo_ boiler. SIMONE similarity $81 \%$. . . . . . . . . . . . . 50

5.6 A Type 2(renamed model clone) chart clone, both (a) and (b) in the ex_mg_ hisl_0062 model. . . . . . . . . . . . . . . . . . 50

5.7 A Type 3(near-miss model clone) chart clone, Fixed-Point Graphical Functions in sf_fxptgf model and Recursive Graphical Function Demonstration

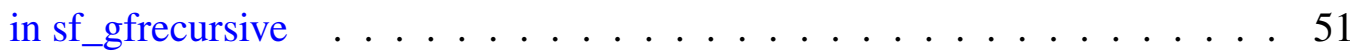

6.1 Contextualization: putting the Stateflow model in Simulink model for the purpose of letting the clone detector analyze the big picture to improve the

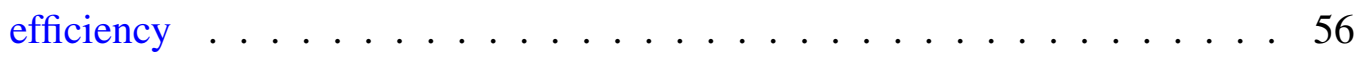


6.2 Graphical presentation of Simulink model with Stateflow: "power_window_ control_system" system consists of blocks, subsystems and "control" Stateflow block in powerwindowlibsa.mdl. . . . . . . . . . . . . . . 57

6.4 SIMONE can detect system "fp_verify_currentdetect_obstacle" and "Mixing \& Combustion" was a clone pair and the similarity of this clone pair was $82 \%$ before contextualization. . . . . . . . . . . . . . . 64

6.5 The different charts in system "fp_verify_currentdetect_obstacle" and "Mixing \& Combustion". . . . . . . . . . . . . . . . . 65

6.6 SIMONE can detect system "fp_verify_currentdetect_obstacle" and "power_ window_control_system/detect_obstacle_endstop/detect_obstacle" was a clone pair and the similarity of this clone pair was $71 \%$ before contextualization. . 66

6.7 SIMONE can detect system "powerwindow" and "power_window_control" was a clone pair and the similarity of this clone pair was $82 \%$ after contextualization as they contain the same chart. . . . . . . . . . . 67

6.8 System "Intake Manifold" in sldemo_fuelsys.mdl. . . . . . . . . . . . . . . 69

6.9 System "emldemo_process_signal" in emldemo_process_signal.mdl. . . . . 69

6.10 System "sf_fxptprecision", which is a Fixed-Point multiplication using Stateflow, in sf_fxptprecision.mdl. . . . . . . . . . . . . 70

6.11 System "sf_array", which is a Stateflow vectorization, in sf_array.mdl. . . . 70

6.12 SIMONE can detect system "Length Adaptation" and "sf_variable_size_data" was a clone pair and the similarity of this clone pair was $76 \%$ before con-

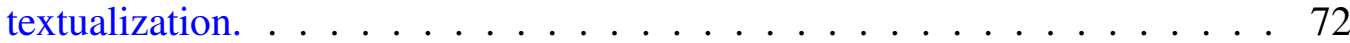


6.13 SIMONE can detect system "sf_resolve_signal_object" and "doc_resolve_ signal_object" was a clone pair and the similarity of this clone pair was $100 \%$ after contextualization. . . . . . . . . . . . . 73 


\section{List of Code Listings}

4.1 State labels general format . . . . . . . . . . . . . 37

4.2 Transition labels general format . . . . . . . . . . . . . 37

4.3 Result of state 4 label after splitting . . . . . . . . . . . . . . 38

4.4 Result of transition 29 label after splitting . . . . . . . . . . . . 38

5.1 Example snippet of the extracted fragment used by Stateflow to store graphical models. . . . . . . . . . . . . . . . . . . . . . . . 44

5.2 Example snippet of sf_aircraft_screen_library . . . . . . . . . 52

5.3 Example snippet of Hydraulic Monitor of sf_aircraft_screen_library. . . . . 53

6.1 Example snippet of the extracted fragment used by Stateflow to store graphical models. . . . . . . . . . . . . . . . . . . 58

6.2 Example snippet of the extracted fragment used by Stateflow to store graphical models. . . . . . . . . . . . . . . . . . . . . 58

6.3 Example snippet of the contextualized fragment of "power_window_control_ system" system in in powerwindowlibsa.mdl. . . . . . . . . . . . . . . 60

6.4 Example snippet of the one line contextualized fragment of "power_window_ control_system" system in in powerwindowlibsa.mdl. . . . . . . . . . . . 68 
6.5 Example snippet of the weighted lines contextualized fragment of "power_ window_control_system" system in in powerwindowlibsa.mdl. . . . . . . . 70 


\section{List of Tables}

5.1 Initial results of the Stateflow model clones found in the Matlab demo set. . 43

6.1 Initial results of the contextualization Stateflow model clones found in the Matlab demo set. . . . . . . . . . . . . . . . . . 62 


\section{Chapter 1}

\section{Introduction}

Model Driven Engineering (MDE) has become popular in industry as a way to build and maintain complex modern software. It is increasingly common to develop software using model-based methodology in embedded software, particularly in areas where risk to life or property is an issue, such as the automotive sector. Simulink ${ }^{1}$ is a modeling language that has been widely used in the development of automotive embedded systems. One component of Simulink is Stateflow ${ }^{2}$, an environment for modeling and simulating combinatorial and sequential decision logic based on hierarchical state machines (i.e. state charts [15]) and flow charts. Developers use MathWorks Simulink/Stateflow and model-based design to develop system models, verify designs using stimulation, and generate production codes in industry.

\subsection{Motivation}

In code-based software development, "Clones are segments of code that are similar according to some definition of similarity". Ira Baxter presented this statement at the First

\footnotetext{
${ }^{1}$ www.mathworks.com/products/simulink

${ }^{2}$ www.mathworks.com/products/stateflow
} 
International Workshop On Detection of Software Clones(2002, Montreal) to define software clones. The simple reuse of code segments by copy \& paste can cause code clones. Koschke [23] detailed the root causes of code clones, and Roy and Cordy[32] classified these reasons into four categories: development strategy, maintenance benefits, overcoming underlying limitations, and cloning by accident. Software clones have a negative effects on maintenance [22]: inconsistent changes to the duplicated same functionality code at multiple locations within the code base can lead to unexpected behavior and faults. Thus, the ability to identify duplicate artefacts in software is important[2].

In model-driven development environments, software clones also happen if there is not an appropriate reuse mechanism. Copy-paste-modify is a typical source of clones in Simulilnk model development[9]. Model clone detection has been an active research area for years. Several researchers[13, 14, 27, 28] have successfully presented their approaches to identify the duplicated parts of models. There are still challenges for finding nearmiss clones in industrial practice, particularly in Matlab/Simulink models. SIMONE[2], a new model clone detection tool, was developed to identify near-miss subsystems clone in Simulink models.

The potential impact of identifying redundancy at the higher levels of abstraction provided by models makes clone detection in models important since identifying redundancy can help in testing design consistency and completeness before implementation. As Stateflow has become increasingly important in Simulink models for the automotive sector, and no researcher has explored the model clone detection in Stateflow models, thus, we extend previous work[2] on clone detection of Simulink models to Stateflow components.

This work is part of model pattern engineering project, one of Network on Engineering Complex Software Intensive Systems for Automotive System(NECSIS) ${ }^{3}$ projects, which

\footnotetext{
${ }^{3}$ www.necsis.ca
} 
purpose is discovery, cataloguing, and formalization of sub-model patterns in automotive models. This project will not predicate what the patterns may be in general. However, this project is to discover patterns that are specific to the domain and the client. Using clone detection technologies to discover a catalogue of repeated sub-patterns is a feasible way. The result of clone detection, clones classes, can provide an initial approximation to the pattern set. Our work is in phase one of the larger project, and the goal is to discover and identify common sub-model patterns in a lager example model set obtained from our industrial partners at General Motors.

\subsection{Contributions}

In this thesis, we make three new contributions: a definition of Stateflow model clones, a new clone detector to support it, and a method to enhance SIMONE[2] with Stateflow information.

First, we present a definition of model clones in Stateflow models, which is the first definition specific to Stateflow models. We also demonstrate an extension to the existing Simulink clone detector SIMONE to include a new class of mode clones that allows SIMONE to detect the potential clones of Stateflow.

Second, we implement the proposed extension for identifying near-miss clones in Stateflow models and conducted and experiment on the Matlab Simulilnk Demo set.

Third, we demonstrate a means of using Stateflow clones to improve detections of Simulink clones. 


\subsection{Thesis Outline}

This thesis is divided into seven chapters that present the new approach of model clone detection in Stateflow models.

Chapter 2 gives the background knowledge needed to understand our approach. This chapter starts with an introduction of Stateflow. It continues with an overview of source transformation systems, particularly TXL[8], and focuses on the aspects of TXL grammar that are used to parse the Stateflow model files. It presents a description about the clone detection and SIMONE, the clone detector we used to identify the similar models. The chapter finishes with a short overview on related work in this field.

Chapter 3 gives a brief introduction of our approach, using examples to illustrate the model clones in Stateflow models.

Chapter 4 describes a pre-processing phase we used in order to extend SIMONE to detect model clones in Stateflow models. This phase is called presentation transformation. The chapter provides a description about the structure of the Stateflow model and the process of folding and label splitting needed for this stage.

Chapter 5 describes the extraction and normalization phase in more detail, using running examples to show the changes of each step.

Chapter 6 describes the aspects of contextualization, which puts the Stateflow models in Simulink models in order to let the clone detector analyze the big picture to improve the efficiency of SIMONE. The chapter starts with a discussion of the relation between a Simulink block and a Stateflow chart. It presents two scenarios to embed the Stateflow chart into its parent block.

Final conclusions and an overview on possible future work is given in chapter 7. 


\section{Chapter 2}

\section{Background}

This chapter gives the background knowledge needed to understanding our work. We begin by discussing Matlab/Stateflow models followed by an overview of a TXL source transformation system. Then we discuss clone detection and the SIMONE clone detector used in our research.

\subsection{Stateflow Models}

MATLAB ${ }^{1}$ is an interactive computing environment and high level programming language. An important feature of MATLAB is extendability. It can be extended with additional toolboxes (Toolbox). Simulink ${ }^{2}$ is one such toolbox. It provides a block diagram environment for designing, modeling and simulating reactive systems. Simulink models can consist of combinations of Simulink blocks, toolbox blocks, and Stateflow blocks. A Stateflow block is a finite state machine within a Simulink model.

Stateflow ${ }^{3}$ provides an environment for modeling and simulating combinatorial and sequential decision logic based on hierarchical state machines (i.e. state charts [15]) and flow

\footnotetext{
${ }^{1}$ www.mathworks.com/products/matlab

${ }^{2}$ www.mathworks.com/products/simulink

${ }^{3}$ www.mathworks.com/products/stateflow
} 
charts. Stateflow produces Simulink blocks, takes the input from Simulink and produces the outputs to Simulink. Figure 2.1 shows how Stateflow integrates with Simulink. A simple Simulink model consists of a Sine Wave block, a Scope block, and a single Stateflow block.

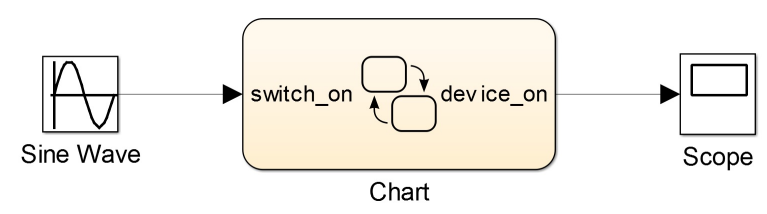

Figure 2.1: A simple example of a Stateflow block within a Simulink model

Complex Simulink models are made of numbers of basic building blocks which are connected with each other. Stateflow is one type of Simulink building blocks, and itself also has its own basic components, which include States, Transitions, History junction, default transition, connective junction, truth table function, graphical function, MATLAB function, box, Simulink function, and History junction. The above basic components are graphical objects in Stateflow and they can be drawn in a chart to create the state transition diagrams, flow charts, state transition tables, and truth tables. Thus, a finite state machine can be graphically represented by transition diagrams.

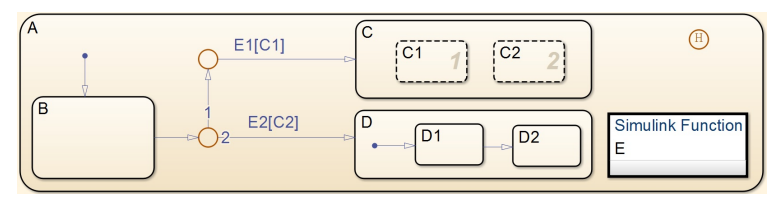

Figure 2.2: A simple Stateflow example. The chart is the parent of the state A, state A is the parent of the B, C, and D states. Exclusive state D1 and D2. Parallel state $C 1$ and $C 2$. The chart also includes a history junction and a Simulink function, and transitions junctions

A set of graphical and nongraphical objects and the relationships between those objects form a typical Stateflow chart. Figure 2.2 shows a simple Stateflow model containing 
mostly basic building blocks. Each block does not have an explicit purpose; it only presents a typical Stateflow model's structure. A state can use a hierarchy to express more complex models, -the example in Figure 2.2 shows three levels of hierarchy. State $A$ is an outer state(or superstate); state $B$ is a substate(or child). State $A$ is the parent of the state $B, C$, and $D$. Every super state has a decomposition, which can be exclusive(state $D 1$ and $D 2$ ) or parallel(state $C 1$ and $C 2$ ). When state $C$ is active, then both state $C 1$ and $C 2$ are active. If state $D$ is active, then either state $D 1$ or $D 2$ is active.

In Matlab, all Simulink and Stateflow models are stored in model files with mdl extension. A model file is a structured text file that contains blocks of text nested in braces simply with key-value pairs that describe all the properties of the model. The file start with the Simulink model, all elements are combined into a single Model section. All elements are stored in hierarchical order. Figure 2.3 shows an example of MDL file textual representation. The Model section includes all the Simulink model elements and the model parameters, configuration set, and configurations references. The BlockDefaults section includes the default settings for all blocks in the model. The AnnotationDefaults section includes default settings for annotations in the model. The System section includes parameters that describe each system and subsystem in the model. The subsystem is nested in the parent system. Each system section contains blocks, lines, and annotations. Stateflow model is represented as a single block in the Simulink model textual representation.

The Stateflow model is stored at the end of the same .mdl file, and all Stateflow elements form a single Stateflow block with linear structure. The textual representation of Stateflow model does not follow the Simulink subsystem representation. That is, the description of sub states are not nested within the description of super states. Figure 2.4 shows an excerpt of the textual representation of a Stateflow model, which in turn is embedded in 


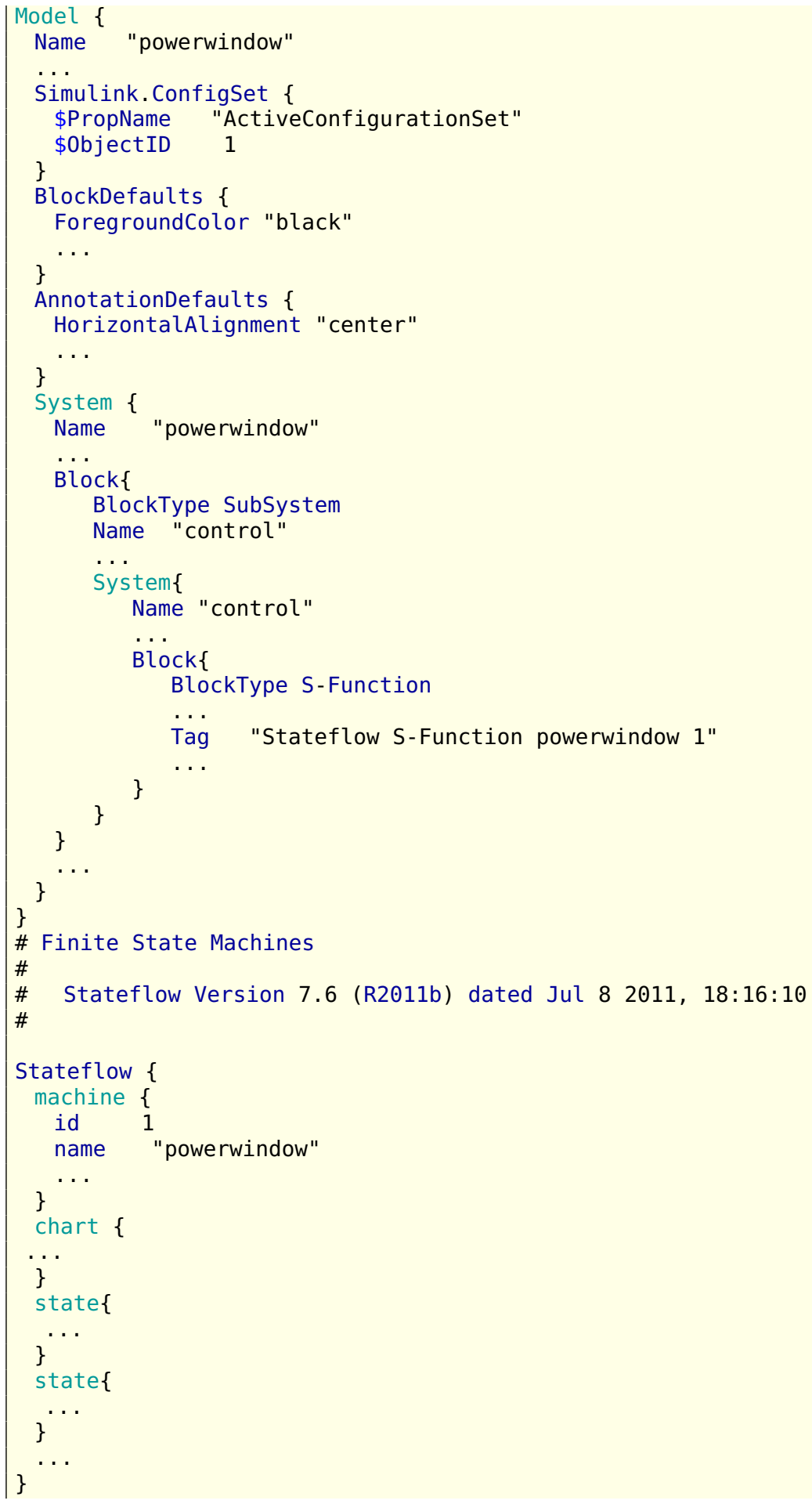

Figure 2.3: Example snippet of the textual representation in MDL file 


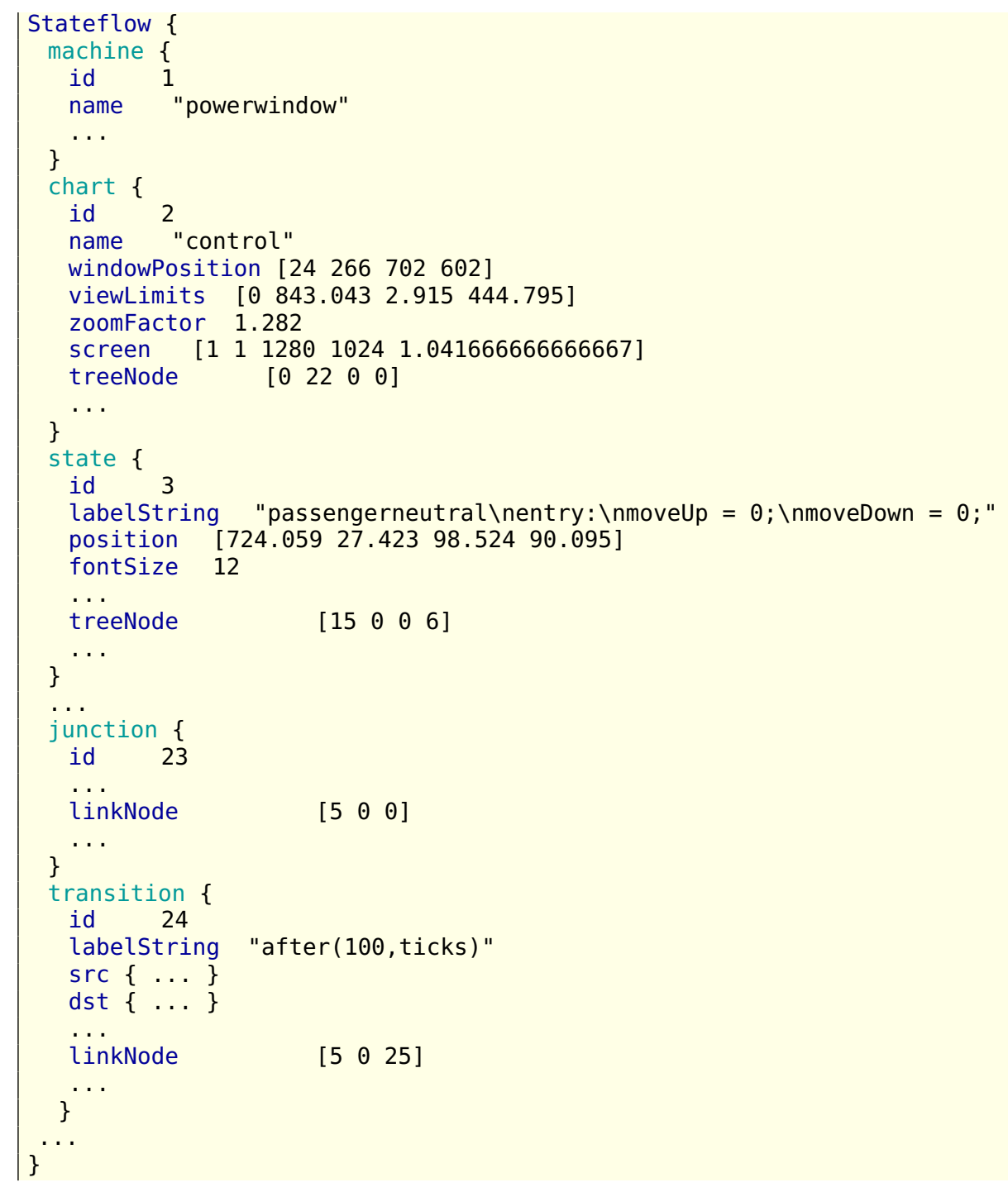

Figure 2.4: Example snippet of the textual representation used by Stateflow

a Simulink model .mdl file. We take this as the text input to our approach. As the listing shows, each item (i.e. state, transition, or junction) is stored sequentially, independent of the inherent hierarchy in the model, i.e. the text form does not reflect the hierarchy of the model graphical representation. 
A Stateflow model's structure is clearly presented by a set of graphical objects. Those graphical objects can be compared to the components of programs in conventional programming languages. Thus, identifying the duplicated graphical objects will help us find clones in Stateflow model.

\subsection{Source Transformation}

In software engineering, source transformation refers to the process of changing one source program into other target forms or languages. Transformations can be performed manually; however it is more common to use a transformation system to automate this process that can accept and apply specifications of the required transformation rules to the source. TXL[8] is a source transformation system designed to support general source transformation tasks. It has proven well suited to a wide range of software maintenance and reengineering tasks. Cordy[10] shows the various examples of TXL's applications in software engineering. TXL has already been used to develop SIMONE[2], and we also use the same technology to extend SIMONE to enable the analysis of Stateflow models.

In our situation, the textual representation of Stateflow models has a flat structure, so it was necessary to transform or restructure it and gather all elements of a state into one state unit. We use TXL to transform the input flat Stateflow model textual representation to a desired form. Also, the extension of SIMONE plugins, the Stateflow detector, was developed in TXL.

\subsubsection{The TXL Source Transformation Language}

TXL[8] is a type of functional programming language designed to support source to source transformation. It has been widely used in software analysis and source transformation 
tasks. TXL program requires a user-defined context-free grammar as part of input to parse the source. TXL generates a parse tree from the source documents based on the pre-defined grammar. Then TXL creates the new parse tree to generate the output based on the rules, and unparses the output to make a document based on the provided grammar.

A TXL program has two components: a BNF(Backus-Naur Form) grammar and transformation rules. The grammar describes the structures of the input to be transformed. The rules specify the output form by source transformation that apply to the input.

\subsection{Clone Detection}

In software development environments, programmers always reuse existing robust sections of code, functions, or procedures instead of working multiple times to leverage their initial efforts. Software code fragments improperly reusing, cut-copy-paste-adapt, may lead to bug propagation and serious maintenance problems[29]. Researchers have developed tools[31] to identify these duplicated code fragments, known as code clones. Clone detection is a process of finding similar or identical code clone based on some measure of similarity.

Generally, researchers[7, 23, 32] categorize the clones into the following types:

Type I (exact) Identical code fragments except for variations in white space and comments.

Type II (renamed)Structurally/syntactically identical fragments except for variations in identifiers, literals, types, layout and comments.

Type III (near-miss) Copied fragments with further modifications such as statements insertions and deletions in addition to variations in identifiers, literals, types, layout 
and comments.

Type IV (semantic)Two or more code fragments that are functionally similar but textually different.

Structural clones Software similarity can be compared with various granularity. Basit et al.[6] detect software similarities at design-level. They identify the recurring patterns of simple clone.

Functional clones The clones that are restricted to the granularity of a function or procedure. Function clones[5] are a subset of structural clones.

Model clones Model clone detection involves identifying identical or similar model fragments.

\subsubsection{SIMONE}

SIMONE[2] is a model clone detector designed for detecting near-miss submodel clones in Simulink models. SIMONE extends the code-based clone detector NiCad[11], which is a TXL-based text comparison software clone detection system with a plugin architecture. NiCad has been successfully used for finding cloned codes in a range of languages, including C, Java, Python, C\#, and WSDL[25]. SIMONE extends the NiCad code clone detector engine to analyze the internal textual representation of Simulink MDL files.

SIMONE extracts all potential clones at a specified level of granularity from the Simulink MDL files as clone candidates. Then SIMONE normalizes the clone candidates by using three plugins: filtering, sorting, and renaming to eliminate any unwanted differences to make the comparison process more precise and accurate. Last, SIMONE takes the filtered, 
sorted, and renamed version of clone candidates as input to compare them line-by-line using an efficient implementation of the Longest Common Subsequence algorithm[17]. This algorithm generates a new sequence of the lines from two potential clones, which contains the longest in-order sequence of lines that two candidates have in common. Then SIMONE computes a percentage of unique items for each potential clone and use the number of unique lines in each as a measure of similarity. If the percentage of unique items in both line sequences of potential clones is zero or below a given threshold, the pair is considered to be clones.

After the comparison, SIMONE produces a series of HTML and XML clone result reports. Two of most important reports are clone pairs and clone classes. The report of clone pairs contains the difference in number of lines and the similarity index for each pair at or above a specified threshold. The report of clone classes clusters all similar clone pairs into clone classes. Each clone class contains all similar clones with difference in number of lines and the similarity index at or above the specified threshold.

\subsection{Related work}

While code clone detection has been extensively researched [31], research on model clones identification has received less attention [13]. Thus far, a few researchers have tried to find the clones in UML behavioural models and Matlab/Simulink models.

Liu et al. [24] proposed a suffix-tree based algorithm to identify duplications in UML sequence diagrams. They converted the 2-dimensional sequence diagram to a 1-dimensional array and constructed a suffix tree from the 1-dimensional array. Their approach identified the common prefixes in the suffix tree and ensured that the duplications detected are extractable and reusable sequence diagram as refactoring candidates. 
Antony et al.[4] proposed an approach for identifying near-miss interaction clones in reverse-engineered UML behavioural models. They used a text-based technique and worked on the level of XMI. Their approach transformed the XMI sequence diagram serialization into a contextualized form and extracted the sefl-contained units of behavioural interaction as clone candidates. A standard code clone detector is applied to identify cloned behavioural interactions from the large set of contextualized textual representation.

Störrle [38] proposed an approach to identify clones in UML models, specifically class, activity, and use case diagrams. The approach is based on model matching and model querying[37]. He implemented the $M Q_{\text {lone }}$ tool to evaluate this algorithm. The tool transforms XMI files, that are generated from UML domain models by using a contemporary UML case tools as input, into Prolog files. Using model matching technique to generate the output from the input model in the query. One advantage of $M Q_{\text {lone }}$ is that using XMI files allows to compare various models from different. Störrle uses a different definition of model clones. His definition requires that the structure of the models are the same and that the labels on each of the model elements are similar. Thus, his approach identifies Type 1 and Type 2 clones, but not Type 3 near-miss clones. He also claims the approach is extendable to Simulink and Stateflow models. However, the approach has not been demonstrated on StateFlow.

The majority of mode clone detection approaches have been tailored for Simulink models[2, 3, 13, 14, 28, 35], and these techniques either use graph based comparison or text-based techniques to do clone detection on Simulink models. None of them has been applied to Stateflow models.

Deissenboeck et al.[14] present one of the first methods to detect the duplication in Simulink models especially in automotive domain. The approach is based on graph theory 
and can detect model clones in Simulink and other graph based data-flow models. In their approach, models are presented as a flattened multigraph where each block and linear connections are normalized by assigning a value. The duplications are checked by performing a depth first search to find matching paragraphs. They implemented their algorithm as a part of the quality analysis framework ConQAT[12] which is publicly available as open source software ${ }^{4}$. Juergens et al.[21] adapted this algorithm to form a clone detection tool chain CloneDetective, which is designed as an open source "workbench for clone detection research" and based on the open source tool ConQAT.

Pham et al.[28] proposed an other graph-based clone detection tool for Matlab/Simulink models called ModelCD, which consists of two algorithms, eScan and aScan. In their approach, the model were represented as a parsed, labelled directed graph and larger clones were identified by adding edges to smaller, already detected clones. The eScan algorithm was designed to detect exact clones achieved by an advanced canonical labelling technique, and the aScan algorithm was designed to exact and approximate clones by computing a vector-based approximation of the structure with a subgraph.

Al-Batran et al.[1] noted that these approaches only consider syntactic clones, so they extended these approaches to cover semantic clones that may have similar behaviour but different structure by using the pattern-based normal-form approach, which normalized model graphs using the models semantic information.

Hummel et al.[18] present an index-based algorithm for model clone detection that is incremental and distributable. In their approach, a Simulink/Matlab model was represented as a directed multigraph and the normalization assigned labels to relevant edges and blocks. The canonical label were computed for each subgraph in a clone index, which is a list of all subgraphs have same size. The clone retrieval process merged clone pairs with same size.

\footnotetext{
${ }^{4}$ http://www.conqat.org
} 


\subsection{Summary}

In this chapter, we have presented an overview of Stateflow model, source transformation, and the TXL language. Next, we discussed clone detection, and the model clone detector chosen for this thesis, SIMONE. Finally, we discussed related works, which mainly focus on Simulink models. The overall approach in identifying duplicated models in Stateflow models will be discussed in detail in the next Chapter 3. 


\section{Chapter 3}

\section{Overview}

The goal of this research is to leverage the efficiency and scalability of already existing clone detection tools to detect similarities among Stateflow models. But to do this, Matlab Stateflow models must first be transformed to a form that allows clone detection tools to be used and the existing tools must be adapted accordingly to an evolved version that can accept the Matlab Stateflow models as one of the inputs.

Model clones are different from code clones, as they are based on matching sub graphs. For typical code clone detection, systems can be classes, functions, blocks or statement sequences depending on the desired level of granularity. However, the appropriate divisions in Stateflow model are charts, states, junctions, and transitions, all of which are presented as a nested set of graphs.

This thesis extends the approach our research group used for Simulink in SIMONE [2] and applies clone detection techniques to the textual representation of the Stateflow model. Figure 3.1 shows the three stages of our approach. The first stage transforms the Stateflow textual representation into a hierarchical textual structure as SIMONE's initial input. The second stage, implemented as a SIMONE plugin, normalizes the initial input to remove irrelevant elements and rename irrelevant naming differences to make the process 
of clone identification more accurate. The final stage identifies potential clone candidates and clusters them into classes.

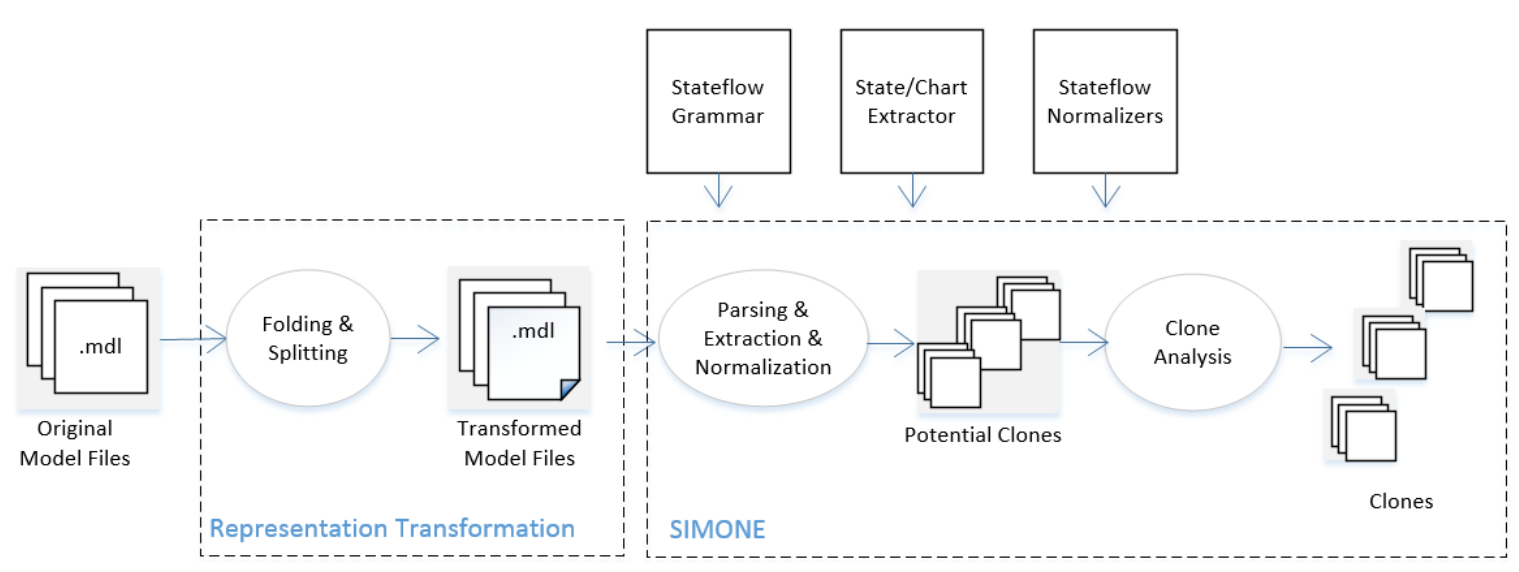

Figure 3.1: Steps of our approach

After Stateflow model clone identification, we also add a contextualization step in order to improve the accuracy of Simulink model clone detection, and that by enabling the identification of model clones in Simulink models that have Stateflow components embedded in them. Figure 3.2 shows the steps of contextualization. The first stage embed the extracted states into the its parents Simulink to form the contextualized model files as SIMONE's initial input. The second stage uses SIMONE to identify potential clone candidates and clusters them into classes.

\subsection{Clones in Stateflow Models}

Software clones are segments of code that are similar according to some definition of similarity [23]. Software clones have an impact on maintenance, and it is important to identify duplicate artefacts [2]. The potential impact of identifying redundancy at the higher levels of abstraction provided by models makes clone detection in models important since it can help in testing design consistency and completeness before implementation. 


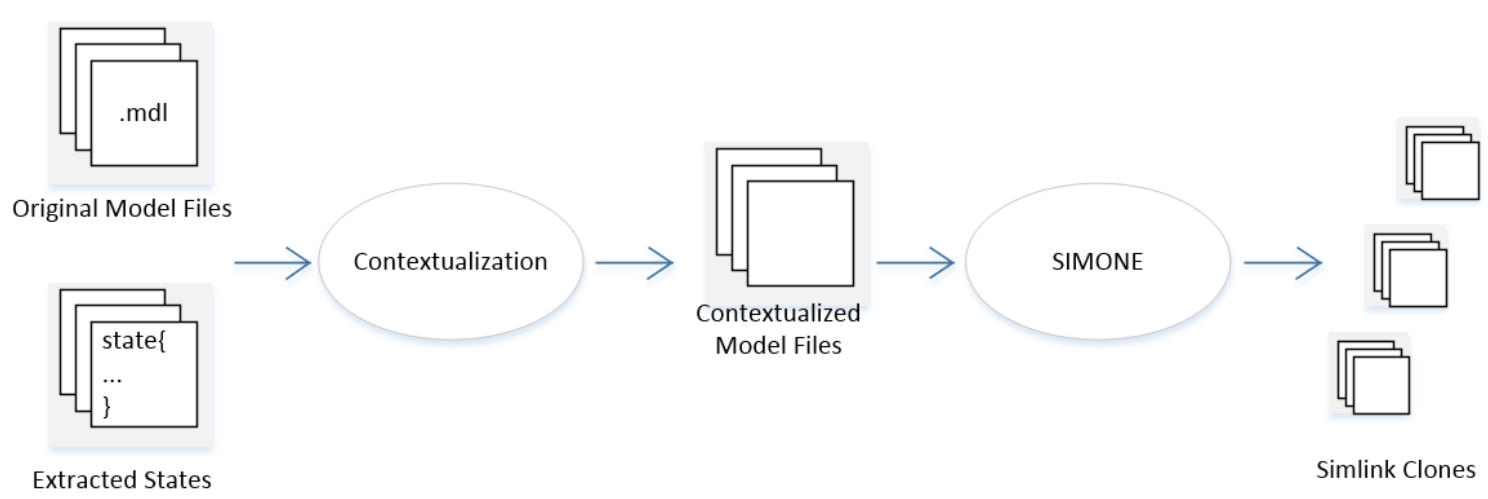

Figure 3.2: Steps of contextualization

Model clones could be defined similarly as the way of code clones are defined. Model clones are similar or identical fragments in software models. However, this is a rather vague definition, because the fragment model could be various. Generally speaking, models are typically represented by graphs, which in turns are represented diagrammatically, such as box-and-arrow diagrams. Model clones are similar subgraphs of these graphs[2]. Thus, the specific definitions given by different researchers are different.

Dessenboek et al. [14] use Matlab Simulink model as an example of model clone definition. Through the relevant attributes of each basic blocks in Simulink model, the Simulink model is converted into the pure mathematical meaning directed labeled graph $G(V, E, L)$, where $V$ corresponds to the blocks; $E$ corresponds to the lines; and $L$ maps vertices and edges to normalization labels. Models are presented as flattened graph without the hierarchy of Simulink. The problem of this method is ignoring the hierarchical structure. In our model pattern project, we try to discrover patterns that have some meaning interms of the structure of models we are working with.

Pham et al. [28] also use Matlab Simulink models. They divided the model clone into two categories: Exact Clone and Similar Clone. Their definition, similar to Dessenboek's, 
convert the model to a directed graph.

Störrle [38] proposes a formal definition of model clone. They introduce the model fragment definition first, as model elements are closed under the containment-relationship. Then define model clone as a pair of model fragments similar to each other. His work includes all types of UML models such as classes or activity diagrams. A fragment can be consisted of each object's properties and its child objects.

In terms of Statelow model clones, we can borrow Störrle's definition. "A Stateflow fragment is a set of graphical object elements that are closed under the containmentrelationship. A Stateflow clone is a pair of Stateflow fragments that have a high degree of similarity between them". The graph elements(states, transitions and junctions etc.) are the fragments of Stateflow model. If the graph elements are similar in a certain structure, then they can be seen as a clone pair. For example two states can be considered clone pairs for each other. Figure 3.3 shows two cloned fragments in two different charts. This simple example can well demonstrate what a Stateflow clone would look like.

\subsection{Clone Classification in Stateflow Models}

An important difference between model clones and code clones is that model clones are based on matching sub graphs. Our research group categorize three model clone types[2] defined as follows:

- Type 1 (exact) model clones are identical model fragments, ignoring variations in visual presentation, layout, and formatting.

- Type 2 (renamed) model clones are structurally identical model fragments, ignoring variations in labels, values, types, and the variations from Type 1. 


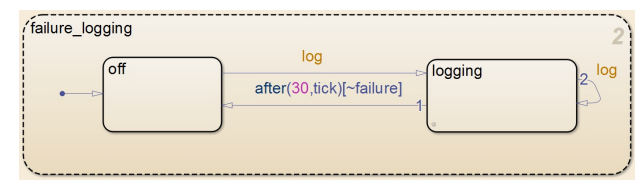

(a) Hydraulic Monitor, (No Filter)/Screen Signals/screen logic

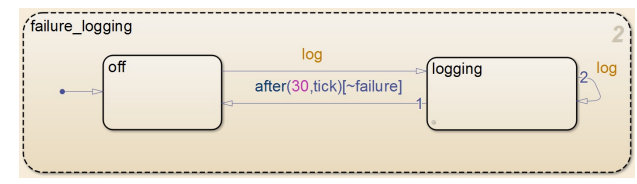

(b) Position Monitor, (No Filter)/Screen Signals/screen logic

Figure 3.3: Example of a clone in a Stateflow model. Both (a) and (b) in the sf_aircraft_ screen_library model and they contain the same number of states and transitions. The two failure_logging states can be considered as the cloned fragments.

- Type 3 (near-miss) model clones are model fragments with further modifications such as small additions or removals of model elements, in addition to the variations from Type 1 and 2 clones.

For our purposes, clones in Stateflow are models that are structurally similar. For example, the same structure states and transitions with different labels, conditions and actions would be considered a clone. There are various techniques and tools for clone detection in program source code [31] such as textual comparison and program dependency graph comparison. Rather than using computationally expensive sub-graph isomorphism to find similar graphs, we extend the approach our research group used for Simulink in SIMONE [2] and apply clone detection techniques to the textual representation of the Stateflow model.

In Simulink, clones are structural in nature and follow natural syntactic boundaries such as subsystems. Stateflow is also represented as a nested set of graphs, but the graphs represent the behaviour of a component in the larger Simulink model as opposed to the structure 
of the model. The addition of Stateflow clone detection to SIMONE serves two purposes. The first is to detect common behavioural elements of the models that are expressed as state machines. The second is by extending the clone detection to Stateflow models, where we can improve clone detection in those Simulink models that contain blocks that refer to the Stateflow models. SIMONE detects clones by considering model elements of the same type, so currently all blocks that encapsulate Stateflow models are also considered similar. We evaluate our approach on a large set of Stateflow models available to us from Mathworks.

\subsection{Stateflow TXL Grammar}

In order to use the existing clone detection tool SIMONE, the first step is to build a Stateflow TXL grammar allowing TXL to parse Stateflow models. We derive a TXL grammar from a large set of Stateflow model examples in the public domain by using iterative grammar techniques[36]. Our grammar identifies all observed elements of the Stateflow models, including machines, charts, states, translations, junctions, events, data, instances, targets and other elements.

\subsection{Representation Transformation}

The first stage of our approach is representation transformation, which helps to identify the artifacts of Stateflow model. This stage performs two tasks. The first task is to transform the linear representation of the model into a nested version that explicitly represents the hierarchy of the model. The second task is to separate the actions of the state from the name of the state, representing each as separate attributes; also include the separation of components of transitions. 


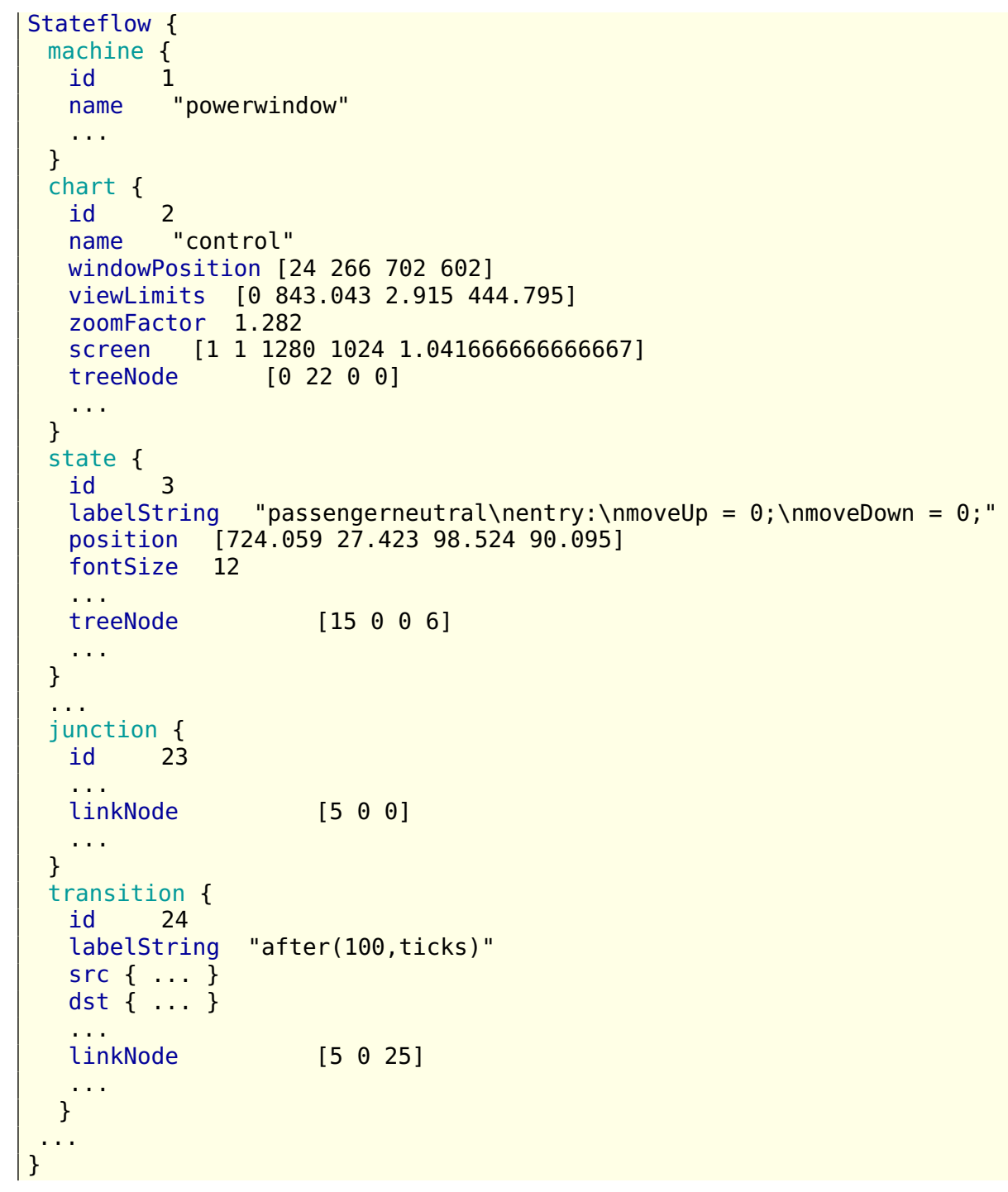

Figure 3.4: Example snippet of the textual representation used by Stateflow

\section{Structure Folding}

Figure 3.4 shows an excerpt of the textual representation of a Stateflow model, which in turn is embedded in a Simulink model file. Each item in the figure (i.e. state, transition, or junction) is stored sequentially, independent of the hierarchy inherent in the model. Instead, 
the hierarchy of the model is represented using the treeNode and linkNode attributes. The treeNode attributes are used in chart and state elements of the model to encode hierarchy by identifying the parent, first child and sibling elements. Thus to handle a Stateflow model, we need to provide an initial transform that moves the textual description of any substates, junctions and transitions and nests them within the description of the parent state.

The transformation takes a folding approach that examines each element in turn and inserts it into the appropriate parent element. At the same time, the nested elements are sorted by type: first states, then transitions, and finally junctions.

Figure 3.5 shows a simplified outline of the new representation. In the figure, the state flow section of the Simulink file contains a single chart, with a single state, that contains a single nested substate and one nested transition. We have omitted the other attributes to emphasize the nested structure.

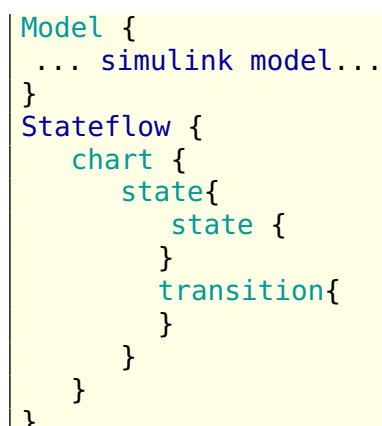

Figure 3.5: Simplified example of folded Stateflow model

\section{Label Splitting}

Figure 3.4 also shows that the state actions are encoded along with the state name into a single string given by the labelString attribute. The state shown in the figure has the 
name passengerneutral as well as an entry action that initializes two variables to zeros. States may have actions associated with the entry and exit from the state, actions that are performed while the state is active and actions that are performed if an event occurs while the state is active. Transition labels are also complex, having triggers, conditions, condition actions and transition actions.

In SIMONE, the potential clones are compared line-by-line. A difference in a single component of a state or transition label renders the entire line different. Thus we split the state labels into the constituent parts, each with its own attribute. The state name, if present, is encoded using a new textlabel attribute. The entry, during and exit actions, when present, are encoded using separate attributes of similar names (entrylabel, duringlabel, and exitlabel). If the author of the state machine model has included formatting such as newlines into the actions, such as in the example in figure 3.4, the actions are split into multiple attributes with the same name. Thus a change in code associated with an entry action will not imply that the during and exit actions of the state are also different.

Figure 3.6 shows the labels generated for the state label in Figure 3.4. The state name has been provided in a textlabel attribute, and the entry actions have been transformed to three entrylabel attributes.

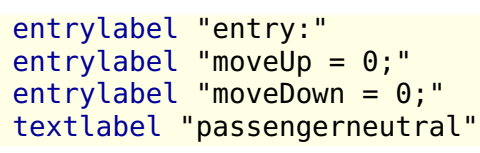

Figure 3.6: Example of a split state label 


\subsection{Extractor Plugin}

A Stateflow extractor is need to identify and extract the clone candidates from the model file containing both Simulink and Stateflow textual presentation. Our extractor for Stateflow provides two granularities of clone candidates. The first level of granularity, chart granularity extracts all of the Stateflow charts as clone candidates. Charts in Stateflow represent entire machines. A Simulink model may have more than one chart, each of which may be instantiated multiple times as blocks in the Simulink Model. The second level of granularity, state granularity, extracts all states in all charts as clone candidates. This allows us to identify cloned state machines that are nested within states.

\subsection{Normalization}

In this stage, we normalize the result of the model files from stage one. The task of this stage includes removing irrelevant elements, renaming elements and sorting elements. These steps can improve the precision and recall of the clone detection phase.

\section{Filtering}

In order to improve the detection of clones, we introduce a filtering plugin similar to the filtering plugin we used for Simulink models in our previous research. This plugin removes the irrelevant elements from the potential clones. Some of these irrelevant attributes are the same as used in Simulink, while others are new. Since there is no published reference for the textual representation of Stateflow models, we are obliged to infer which of the attributes are important and which are irrelevant. Figure 3.4 shows some of these irrelevant elements: the position of each element, the font size used to display labels and the zoom factor of the chart. Although these attributes are important to the human understanding 
of the model, they are not relevant to the semantic meaning of the models. Two models identical when comparing states, transitions, junctions and actions may actually contain differences based on how they are rendered for viewing. The differences in these attributes can overwhelm the similarities in the attributes that carry the semantic meaning of the model.

\section{Renaming}

While filtering improves the similarity factor of the identified clones and help identify more clones similarity of the clones, we found that there are still some clones we could identify manually that are not detected. Some of the values of the attributes represent internal information such as the $i d$ number of a state and are used to build structural information such as the relationships between transitions and states.

We adapted the SIMONE blind renaming plugin to rename the attributes, giving them all the same value. Renaming significantly improved the similarity and also new cloned pairs are found. All detected clones have been checked manually to compare the graphical representation. All clones found in the example set models are valid clones.

\section{Sorting}

The last source of difference in similar models is the order in which the elements are saved to the file. For example, there are two clone models $X$ and $Y$, have the same states $A, B$ and $C$. In model $X$, the order of the states are $A, B$ and $C$. In model $Y$, the order of the states might be $C, B$ and $A$. In Simulink models, we use the Type, Name, Source, Block, and Port attributes as sorting criteria. Unfortunately, Stateflow does not have a name attribute we can use. The solution is to sort the elements by size. Each state may contain a different 
number of substates, junctions and transitions. Therefore, we sort the states by the number of nested elements.

\subsection{Contextualization}

Since Stateflow can be seen as a block within Simulink model, and in order to enhance our SIMONE accuracy of identifying Simulink clones, we investigate explicating the state machines into the parent Simulink model. We call this process contextualization because it adds Stateflow context to its parent letting the clone detector analyze a bigger view. We use three different approaches to examine the contextualization. First, we put the entire state machine back to its parent Simulink. Second, we only try to put a reference line of Stateflow to its parent. Third, we put an average weight of a Stateflow back to its parent.

\subsection{Evaluation}

We tested our proposed Stateflow clone detector, new plugins of SIMONE, on Matlab Simulink models demo set. The set contains 269 Simulink models with Stateflow model in them out of total 3356 Simulink models. These models are publicly accessible and they come with the Matlab installation. Using a threshold of $30 \%$ difference(i.e. at least $70 \%$ of the lines are the same) and a minimal clone size of 100 lines, we were able to extract 1499 states and 339 charts. After normalization, we found 271 state clone pairs clustered in 43 clone classes, and 676 chart clone pairs clustered in 30 clone classes.

We also tested the contextualization method. We run SIMONE on the contextualized model files and this process is a multiple stages clone detection approach. There are total 237 valid Simulink models containing 339 Stateflow charts in the previous model set. Using the same threshold $30 \%$ and clone size of 100 lines, we were able to extract 1388 Simulink 
systems. We applied SIMONE to these systems and found 4100 clone pairs clustered in 24 clone classes. After embedding the 339 charts back to their parents blocks,we found 4902 clone pairs clustered in 42 clone classes in full lines method, 4182 clone pairs clustered in 25 clone classes in one line method, and 5058 clone pairs clustered in 32 clone classes in weighted lines method.

\subsection{Summary}

In this chapter, we have given an overview of the work in this thesis. First, we derive a Stateflow TXL Grammar. Then, we explicate the hierarchical structure of Stateflow by folding and splitting. Next, we normalize our clone candidates. Finally, we examine the combined identification of clones in complex Simulink models that have Stateflow components embedded int them. In the next chapter, we will describe each phase in more detail. 


\section{Chapter 4}

\section{Representation Transformation}

From the previous chapter, we have overviewed the Stateflow model representation at the text level as in figure 3.4, all states, junctions, transitions and other elements are sequentially stored in the Simulink model file. This linear text representation without hierarchical structure of each object is the first challenge for Stateflow model clone detection. But by converting the textual representation to a nested version, we can create suitable model fragments for clone comparison. In this chapter, we will show how to transform the liner representation of the model into a hierarchical version and separate the actions of a state to represent each as separate attributes. Figure 4.1 shows the detailed steps of representation transformation.

\subsection{File Structure}

Both Simulink and Stateflow models are saved in the same model file with .mdl extension, which contains the graphical representation information of a model created in the Simulink graphical editor. The .mdl file is a structured text file that contains blocks of text nested in braces simply with key-value pairs that describe all the properties of the model. If a Simulink model contains a Stateflow block, then all Stateflow elements form a single 

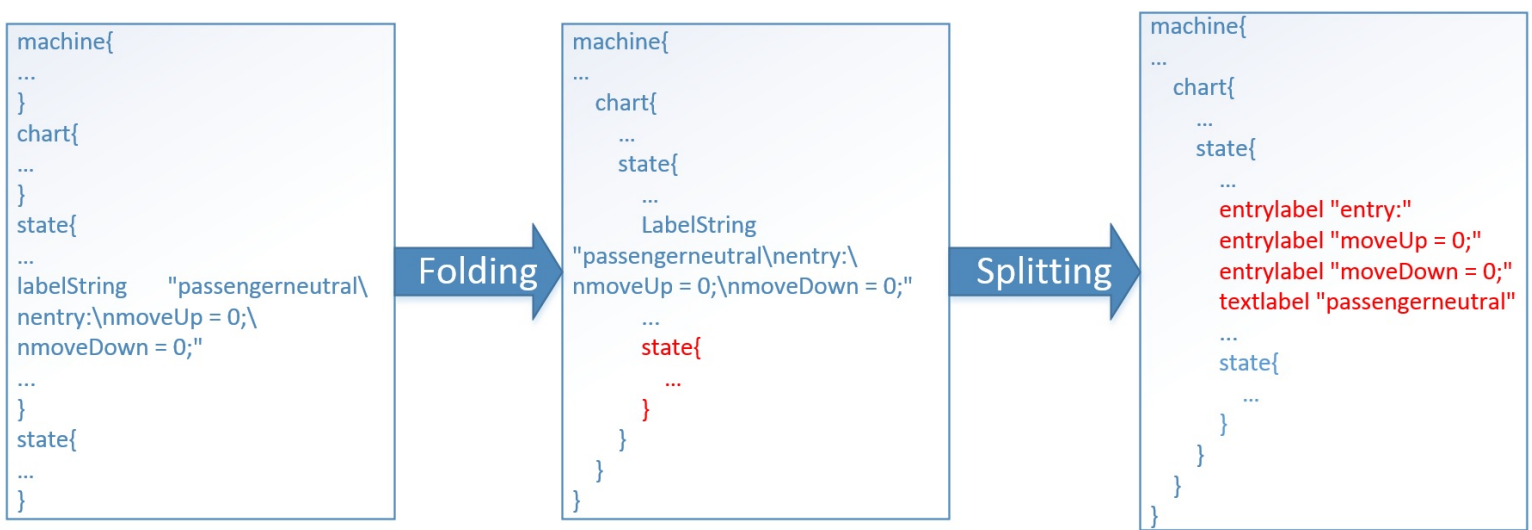

Figure 4.1: Representation Transformation: explicit model hierarchy and separate each action to its own attribute

Stateflow block which is stored at the end of the same .mdl file.

Stateflow block is one of the top blocks in a mdl file started with Stateflow keyword. This top block contains other Stateflow object blocks that describe charts, states, transitions, junctions, data and events. Each Stateflow object block contains all its attributes and values.

Figure 4.2 shows the hierarchical structure of the Stateflow elements. This structure is encoded implicitly in the textual representation of the model using specific attributes will detail later in section 4.2.

Each Stateflow object is assigned a sequential unique number as the $i d$ attribute that is shared across all the Stateflow charts in a Simulink model. The Stateflow machine is the highest object in Stateflow hierarchy, so 1 is assigned to machine in the example of Figure 4.3 example. Machines contain all Stateflow objects. The following object in the hierarchy is chart, which contains state, box, function, data, event, transition, junction, and note objects. The state object may contain all these objects. In the textual representation of Stateflow, object blocks are ordered in a certain way, starting from machine then followed by chart, state, transition, junction, data, event, instance, target. Since the hierarchical 


\section{Stateflow Hierarchy}

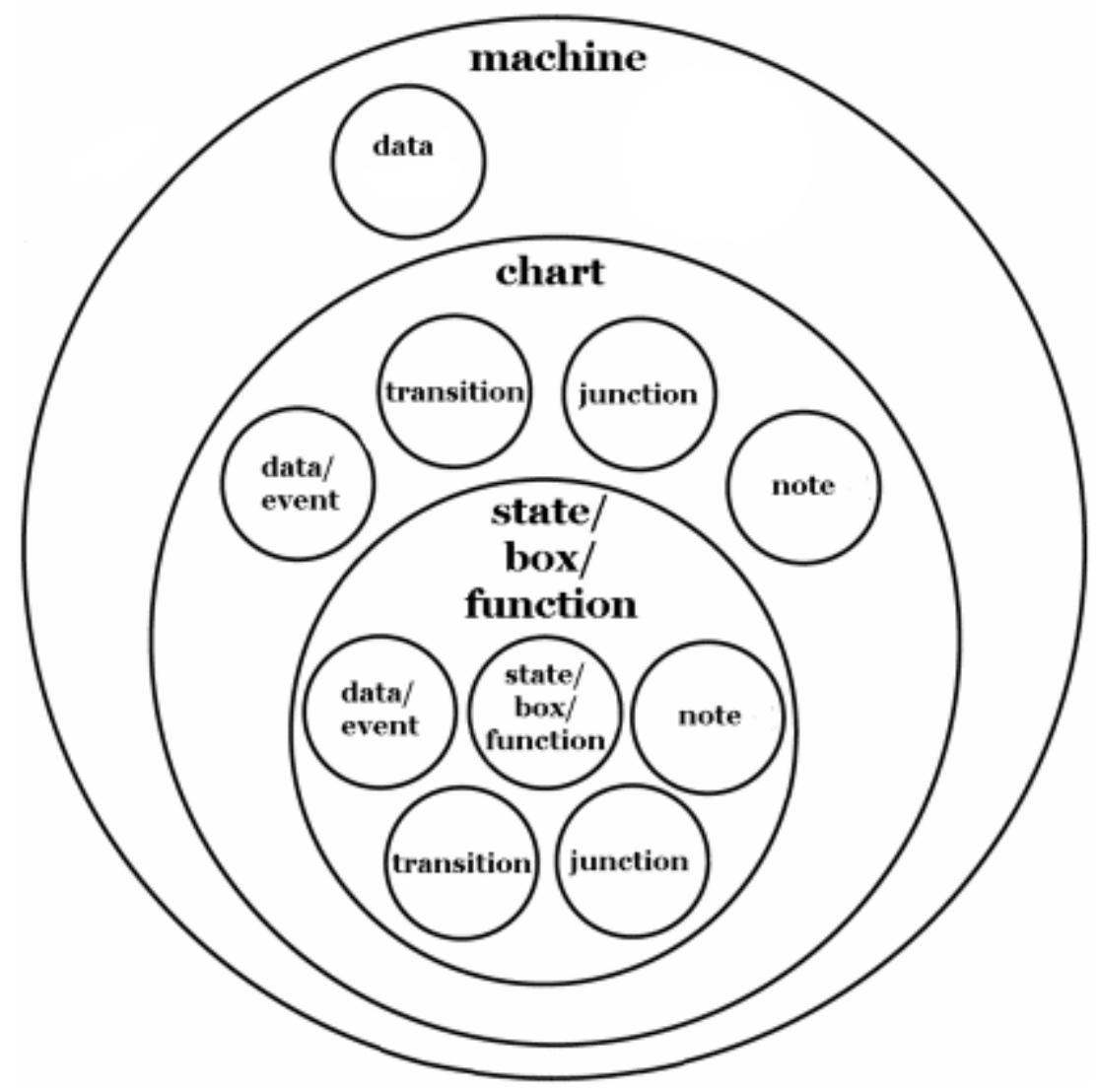

Figure 4.2: Stateflow Hierarchy of Objects[19]

structure of those elements are encoded implicitly in the model and because our text-based clone detection approach requires a well defined granularity, our first challenge is to reveal this hierarchical structure as detailed in the next section.

\subsection{Structure Folding}

Our concise solution is to restructure the textual presentation of Stateflow mdl file to show explicitly the Stateflow hierarchy[19], which folds each object to its parent object to form a nested textual presentation, called Folding. The purpose of folding phase is to bring all 


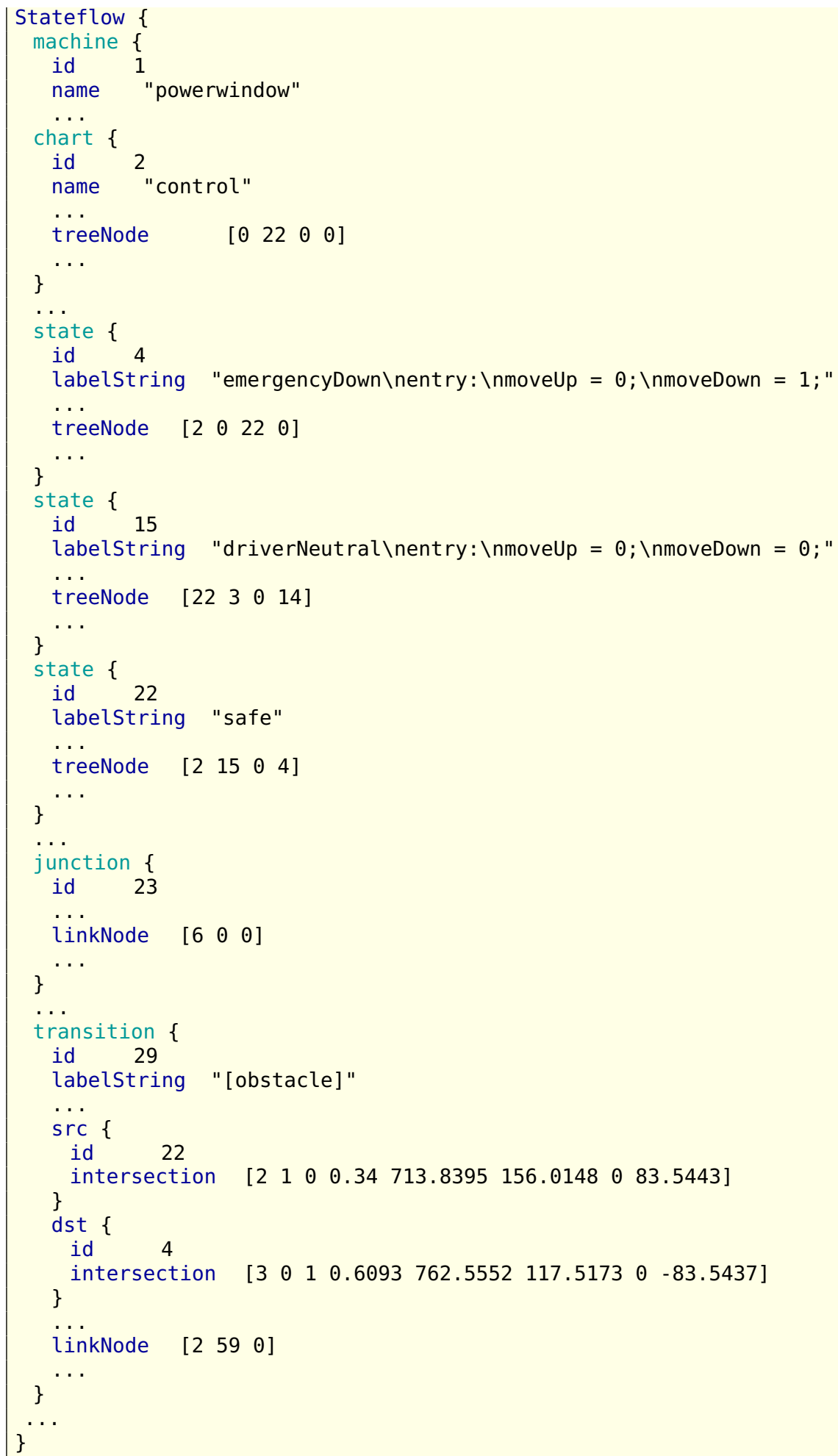

Figure 4.3: Textual Presentation of powerwindow model chart control 


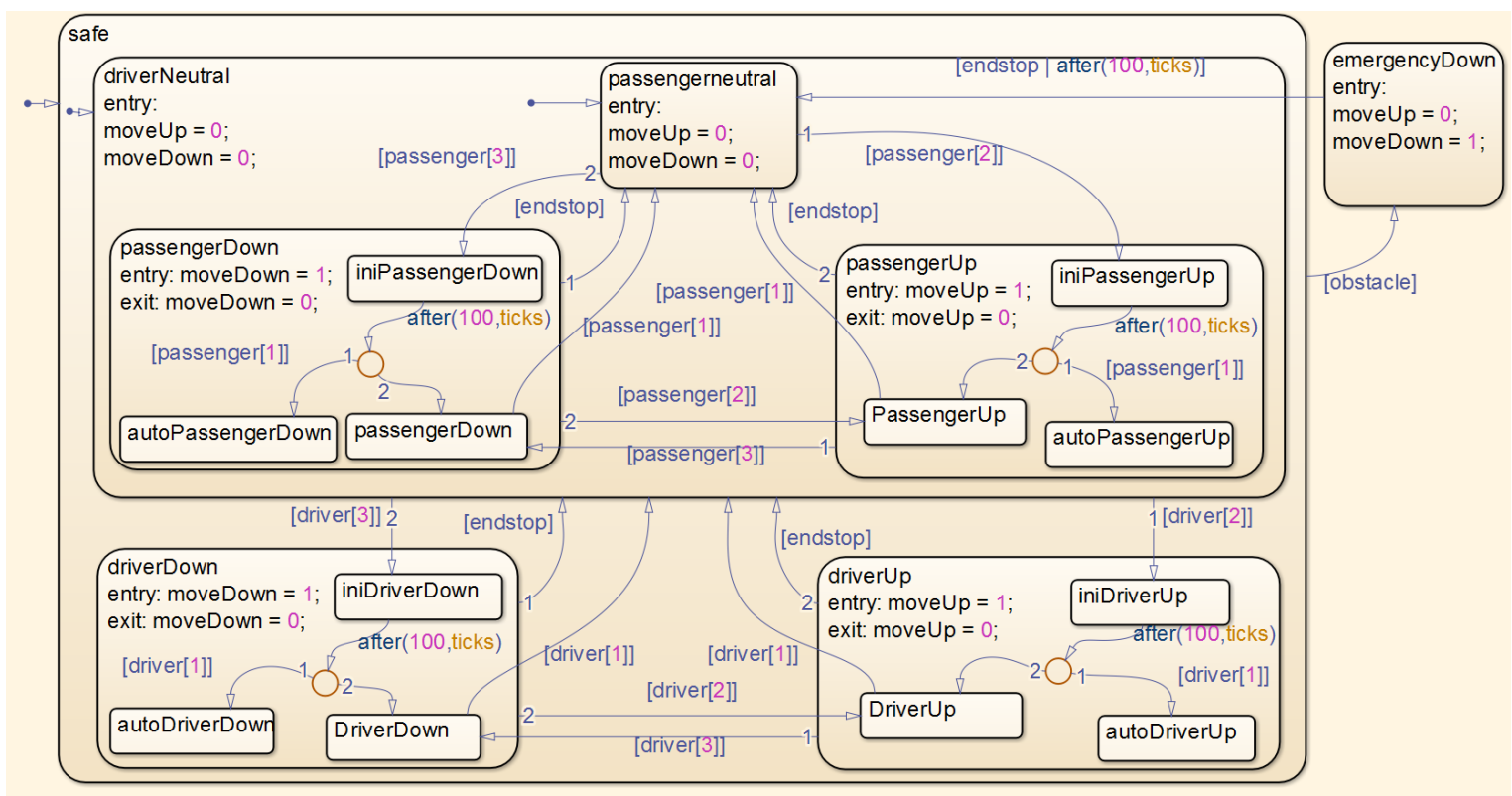

Figure 4.4: Graphical Representation of powerwindow model control chart ${ }^{1}$

the elements referenced by the attributes into a self contained unit so that the all related elements can be extracted as one potential clone fragment by the extractor. This step is similar to Martin et al.[26] and Antony et al.[4]. Martin et al. presented the technique for extracting the elements of each operation in WSDL(Web service Description Language) and consolidating them into a self-contained unit. Antony et al. applied this technique to the XMI text representation to reveal the hidden hierarchical structure of the model and granularize behavioural interactions into conversational units.

From the mdl file structure, we have seen all the Stateflow objects are stored in a sequentially linear text. So how the Stateflow preserves the hierarchical information in the mdl file ${ }^{2}$ ? Figure 4.3 shows the secret. treeNode and linkNode are the key attributes that preserve the hierarchical structure of Stateflow models. We can find treeNode in the chart and state blocks, and linkNode in the junction, transition, event, data and target blocks of

\footnotetext{
${ }^{1}$ Power Window model is from MATLAB demo set.

${ }^{2}$ https://cs.uwaterloo.ca/ aljuarez/mdl2smv.html
} 
.mdl file. The value of treeNode attribute is composed of four integer array that preserves the implicit hierarchy. Each number of the four identifiers represents a Stateflow object. The first number is a reference to a superstate or a parent object. The second one indicates the first child object. The third and the forth are references to the previous sibling and the next sibling. In the textual presentation example Figure 3.4, chart 2 has a treeNode and the value is $\left[\begin{array}{lll}0 & 22 & 0\end{array}\right]$. This chart contains a state with the id 22 which is the state "safe" at the top level of this example hierarchy shows in the Figure 4.4. And the value 0 in treeNode means no Stateflow object is linked. State 22 has a treeNode and the value is [ 2150 4]. This "safe" state has a parent object which is chart 2, and has a child state 15 "driverNeutral" and a next sibling state 4 "emergencyDown". The "safe" state does not have a previous sibling. It reflects the graphical representation in Figure 4.4, state safe has a child "driveNeurtral" and a sibling "emergencyDown". The linkNode attribute is similar to the treeNode in a chart or state, but has only three integer numbers without the the first child. The value only contains the parent, the previous sibling, and the next sibling. In the example, the linkNode value of junction 23 is $\left[\begin{array}{lll}6 & 0 & 0\end{array}\right]$ that means it only has the parent "passengerDown". The junction is in the middle of the "passengerDown" state in Figure 4.4. The linkNode value of transition 29 is [2 590 ] and it has attribute $s r c 22$ and $d s t 4$. So the transition 29 presents a direction line from state "safe" to state "demegencyDown" with label "[obstacle]" in Figure 4.4.

We know where the hierarchy information is, so what we create a transformation rule to fold each child object to its parent object. The .mdl fils are the input for our transformation program written in TXL. The program folds the textual block of any Stateflow objects such as substates, junctions and transitions and put them in the parent block. The transformation 
takes a folding approach that examines each element in turn and inserts it into the appropriate parent element. At the same time, the nested elements are sorted by type: first states, then transitions, and finally junctions.

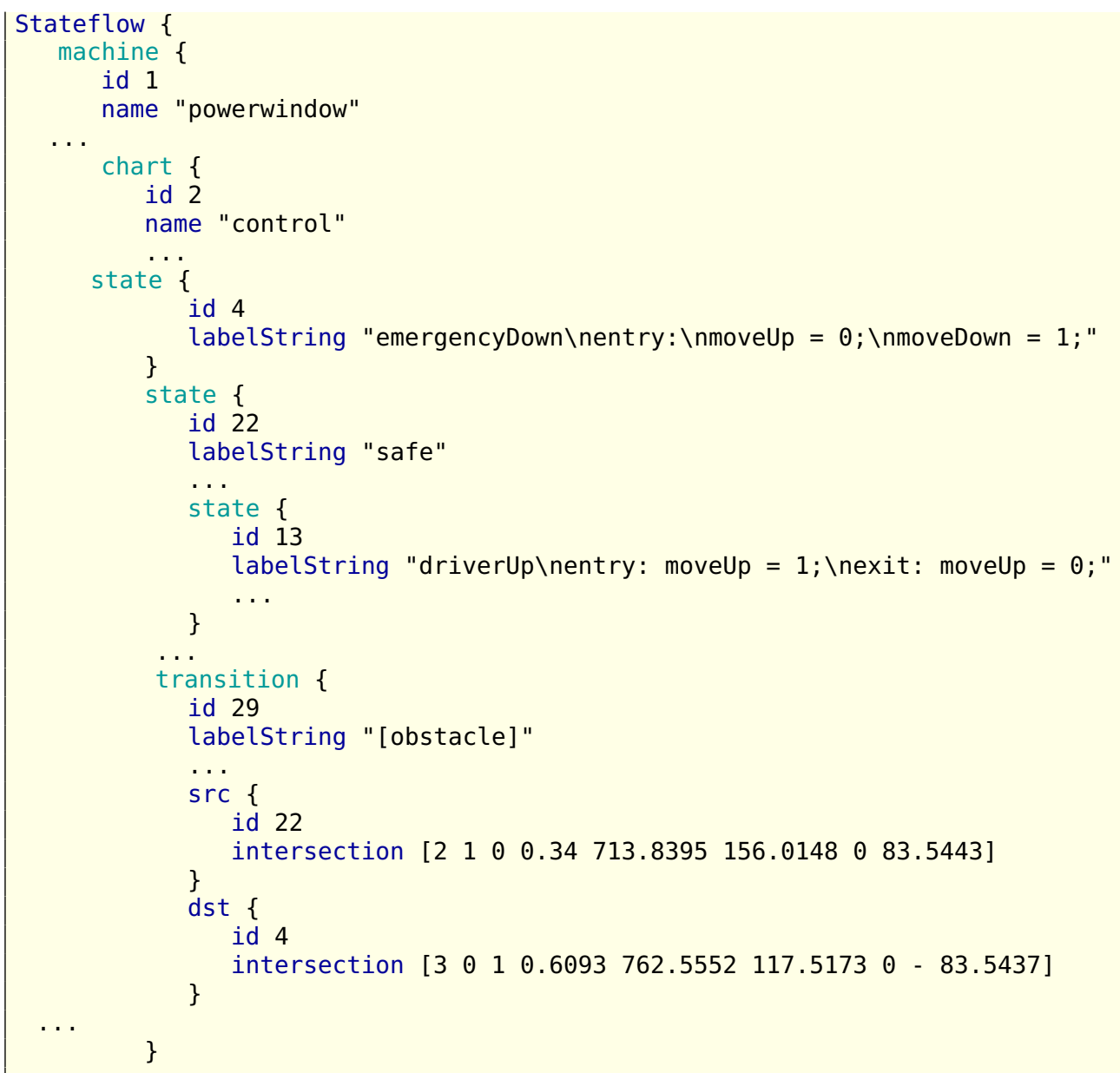

Figure 4.5: Folding textual presentation result of powerwindow model

Figure 4.5 shows a simplified version of the new representation. In the figure, every Stateflow object has been fold to its parent object properly, it clearly shows the nested structure. 


\subsection{Label Splitting}

The labelString is an important attribute for states and transitions. Some of Stateflow properties such as state names, actions and transition conditions are encoded as a single string of labelString attribute. The labelString in a state has a general format shown in Listing 4.1. The first line is the name of a state, and the following lines are a set of actions after each keywords: entry, during and exit. These actions are executed at the different phase of a state, i.e.,entry actions are at the activation of a state; during actions are at the simulation phase; and the exit actions are executed when a state is going to be deactivated. In the example of Figure 4.4, state "driverNeutral" has the name driverNeutral as well as an entry action that initializes variables moveUp and moveDown to zero.

Listing 4.1: State labels general format

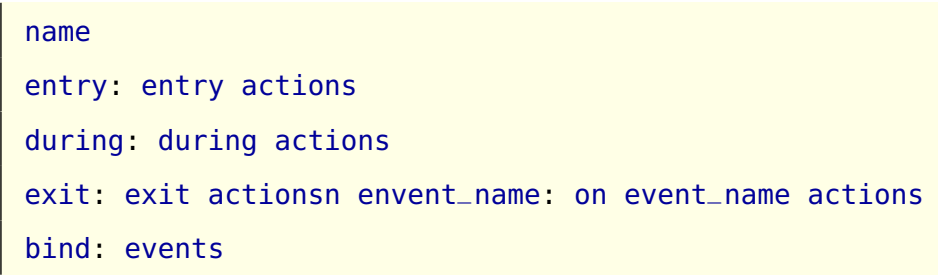

The labelString of transition has different syntax and semantics. Listing 4.2 shows a general format of transition label. Transition labels contain event triggers, conditions, condition actions and transition actions.

Listing 4.2: Transition labels general format | event[condition] \{condition_action\}/transition_action

Our model clone detection tool, SIMONE, is based on comparing line as a whole. So a difference in a single part of a state or transition label renders the entire line different. We separate the single lableString into multiple lines to improve the precision of our model clone detection. We introduce new attributes and split the state labels into several new 
attributes and its own value. The state name, if present, is encoded using a new textlabel attribute. The entry, during and exit actions, when present, are encoded using separate attributes of similar names (entrylabel, duringlabel, and exitlabel). If the author of the state machine model has included formatting such as newlines into the actions, i.e., in the example in figure 4.3 , the labelString of state 4 has three newlines and the actions are split into multiple attributes with the same name. Thus a change in code associated with an entry action will not also imply that the during and exit actions of the state are different.

Listing 4.3: Result of state 4 label after splitting

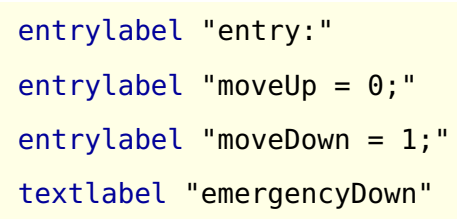

Listing 4.3 shows the labels generated for the state 4 label in the previous example at the top right corner of Figure 4.4. The state name has been provided in a textlabel attribute, and the entry actions have been transformed to three entrylabel attributes.

Transition label has different components with the state labels, so we introduce four new attributes to the textual presentation. We separate each of the components of the transition labels into separate attributes. These components are identified by the new attributes eventlabel, conditionlabel, condition action, and actionlabel. This provides us with finer grained control over the comparisons used for clone detection. For example we can distinguish between a change in an event label from a change to both an event label and the code given by the action of the transition.

Listing 4.4: Result of transition 29 label after splitting

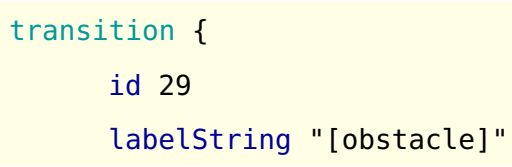




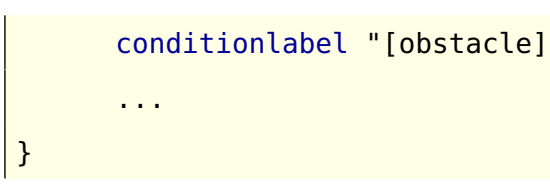

Listing 4.4 shows the labels generated for the transition 29 label in the previous example which connects to the state 4 at the top right corner of Figure 4.4. The transition label does not contain any event, condition action, and transition action, so only conditionlabel attribute and its value has been added in this example.

\subsection{Summary}

In this chapter we have discussed a pre-processing phase, presentation transformation, that we apply to the model files before we run the SIMONE clone detector. We started by describing the mdl file structure, which is the base for doing the transformation. Then we explained how we fold each Stateflow object to its parent and how we split the labels into several single attribute and its value. The following chapter will go over the next stage of model clone detection - the normalization phase in detail. 


\section{Chapter 5}

\section{Extraction And Normalization}

Generally, clone detection tools are hunting for fragments of codes or models to compare as clone candidates, which are extracted from their representation. In order to extract Stateflow model fragments from .mdl files, we need to add a new extractor to SIMONE, which already has a Simulink extractor. After the extraction, the extracted fragments can be normalized in several different ways. The normalization step is necessary becuase it improves the precision and recall of the clone detection phase. In this chapter, we will go over these transformations in detail and give the initial results of our model clone detection. Figure 5.1 shows the details of the normalization transformation.

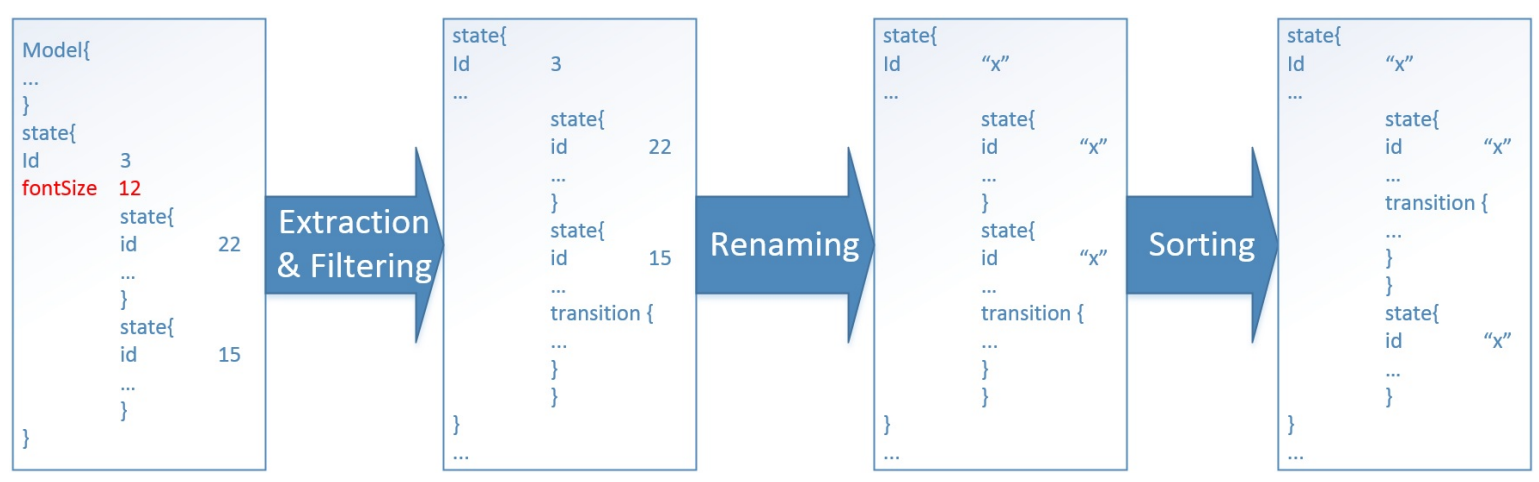

Figure 5.1: Normalization transformation steps: extraction \& filtering, renaming, and sorting. 


\subsection{Extraction}

Identifying and extracting the potential clones are an important process of clone detection. We need to extract appropriate units from the hierarchy of Stateflow, described is figure 4.2, as potential clones. We could use the entire Stateflow block in the mdl file, but that would not provide the comparison of fragments at the state level. That would mean two similar states might be ignored, and only the highest level state machines get compared. To achieve a finer level of comparison fragment, we need to define new granularity in SIMONE for Stateflow.

\subsubsection{Granularity}

A granularity is required to be specified in finding duplication in models, which specifies the level of fragment that should be compared during the clone detection process. SIMONE has three levels of granularity[2]: models, systems and blocks. We provides two granularities of clone candidates for Stateflow. The first, chart granularity extracts all of the Stateflow charts as clone candidates. Charts in Stateflow represent entire machines. A Simulink model may have more than one chart, each of which may be instantiated multiple times as blocks in the Simulink Model. The second level of granularity, state granularity, extracts all states in all charts as clone candidates. This allows us to identify cloned state machines that are nested within states.

\subsubsection{Extractor Plugin}

SIMONE can identify near-miss sbusystem clones in graphical models by adding plugins to NiCad[11, 30, 33] to support Simulink model clone detection. So SIMONE can take NiCad's architecture advantage, which allows adding new plugin as extractor that written 
in TXL. We developed two new extractors to extend SIMONE to support model clone detection for Stateflow models.

All extractors are placed in the /txl directory with a name of the form language-extractgranularity.txl, where language is the name of the language mdl and granularity is the name of the granularity (e.g. charts, states). These extractor modules are used by SIMONE to parse and extract the units specified by the granularity. There is a configuration file contains the setting for SIMONE to look for a file with the name of mdl-extract-charts.txl or mdl-extract-states.txl.

\subsubsection{Clone Detection Results}

We run SIMONE with our new extractor on the set of Stateflow demo models provided by MathWorks version R2011b. There are a total of 269 model files that contain Stateflow in the demo set ${ }^{1}$. Our initial, baseline experiment uses only the candidates extracted at both levels of granularities without any normalization. Using a threshold of $30 \%$ difference(i.e. at least $70 \%$ of the lines are the same) and a minimal clone size of 100 lines, we were able to extract 1499 states and 339 charts and find several clones in the demo set. A clone class is the equivalence class induced by the clone pair relationship. If $a$ and $b$ are clone pairs, and $b$ and $c$ are clone pairs, then $a, b$ and $c$ form a clone class. Table 5.1, the Extractor only column, shows the initial results. We found 205 state clone pairs clustered in 24 clone classes, and 514 chart clone pairs clustered in 27 clone classes.

State "safe" of powerwindow model in Figure 4.4 is one example of our clone detection results at state level. There are in total nine models that contain a "safe" state and they form one clone class with similarity $73 \%$. Unsurprisingly, we also found the same clone class at chart level because the "safe" state almost dominates its parent chart. The chart granularity

\footnotetext{
${ }^{1}$ They come with Matlab installation located at the Matlab installation directory.
} 


\begin{tabular}{|l|c|c|c|c|c|c|c|c|}
\hline \multirow{2}{*}{$\begin{array}{l}\text { Total } \\
\text { states }(1499) \\
\text { charts(339) }\end{array}$} & \multicolumn{2}{|c|}{$\begin{array}{c}\text { Extractor } \\
\text { Only }\end{array}$} & \multicolumn{2}{|c|}{$\begin{array}{c}\text { Filtered } \\
\text { Only }\end{array}$} & \multicolumn{2}{c|}{$\begin{array}{c}\text { Filtered } \\
\text { \& Renamed }\end{array}$} & \multicolumn{2}{|c|}{$\begin{array}{c}\text { Filtered, Sorted } \\
\text { \& Renamed }\end{array}$} \\
\cline { 2 - 10 } & state & chart & state & chart & state & chart & state & chart \\
\hline Clone pairs & 205 & 514 & 151 & 275 & 281 & 728 & 271 & 676 \\
\hline Clone class & 24 & 27 & 20 & 23 & 44 & 27 & 43 & 30 \\
\hline
\end{tabular}

Table 5.1: Initial results of the Stateflow model clones found in the Matlab demo set.

provides an high level similarity comparison as a whole block in the Simulink model, and the state granularity provides a fine level of state similarity comparison.

Further examination of the results reveals models that are identical in the graphical view do not have one hundred percent similarity. At the same time, we also found some states having identical graphical representation do not show up in the clone detection result. If we adjust the threshold a little higher, some missing states will be found in the clone detection report. Obviously, a normalization step is required to improve the precision. The most obvious differences were differences in layout attributes, and normalizing these attributes could improve clone detection. To evaluate the effectiveness of the normalization of the states and charts are necessary to improve Stateflow clone detection.

\subsection{Normalization}

In this phase, three new transforms are implemented in TXL for both states and charts to normalize the result of the model files from the previous extraction step. The three transforms are filtering, renaming and sorting. They follow the same naming convention of plugin file in this format : language-type-granularity.txl, where language is mdl, type is the name of normalization type (e.g. rename-blind, transform-sort) and granularity is the name of the granularity (e.g. charts, states). The filtering plugin is developed as part of extraction. This is conform the process order with SIMONE. 


\subsubsection{Filtering}

Listing 5.1 shows an example of the extracted textual representation for Stateflow with all elements in chart, state, transition and junction. There exist a number of elements (windowPosition, viewLimits, position, fontSize) related to layout and formatting, which have no meaning from the model cloning point of view. Even a small change in an element such as font or position can make identical model fragments look very different when compared in the textual representation level and prevent SIMONE from finding them as clones.

Listing 5.1: Example snippet of the extracted fragment used by Stateflow to store graphical models.

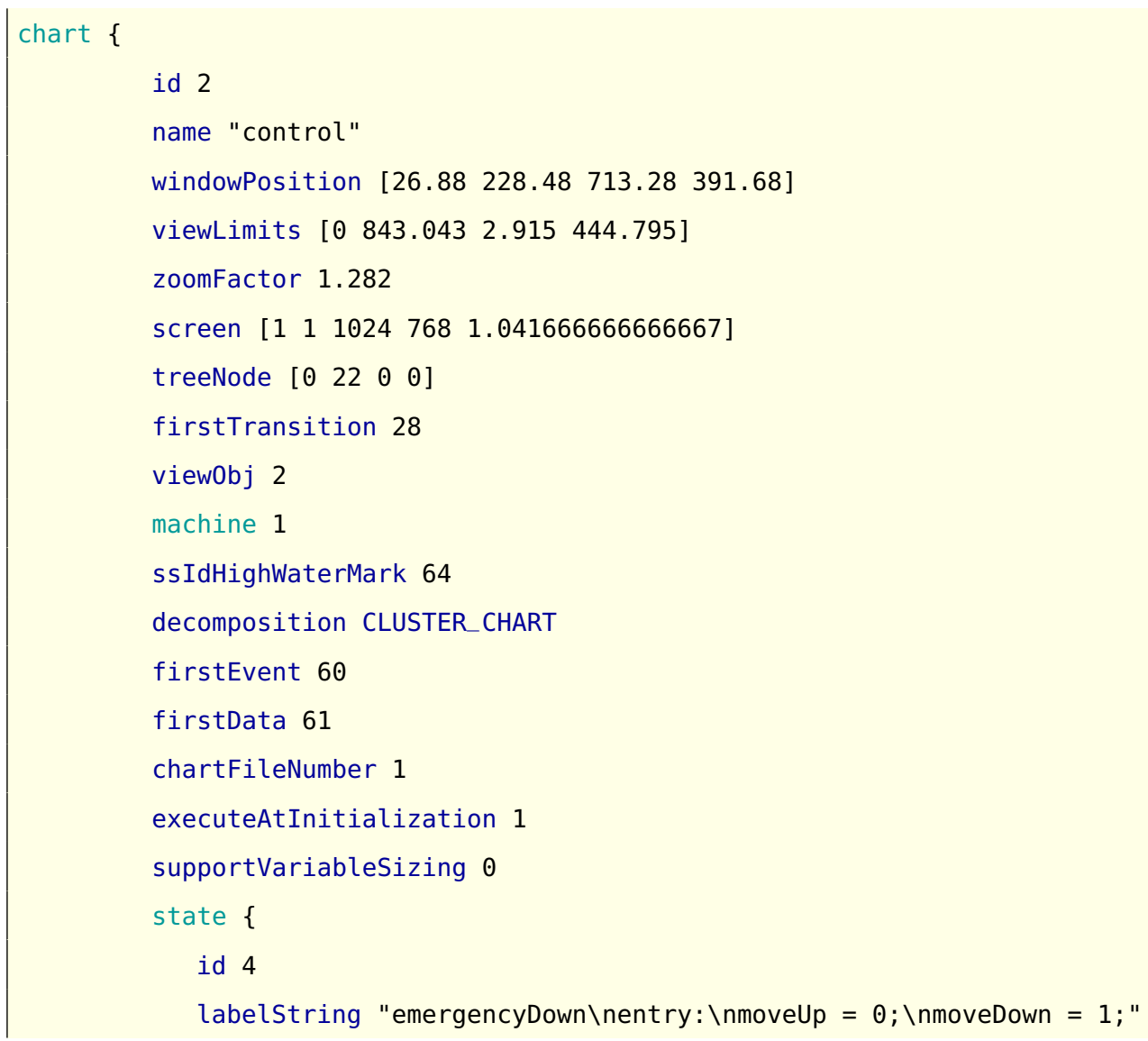




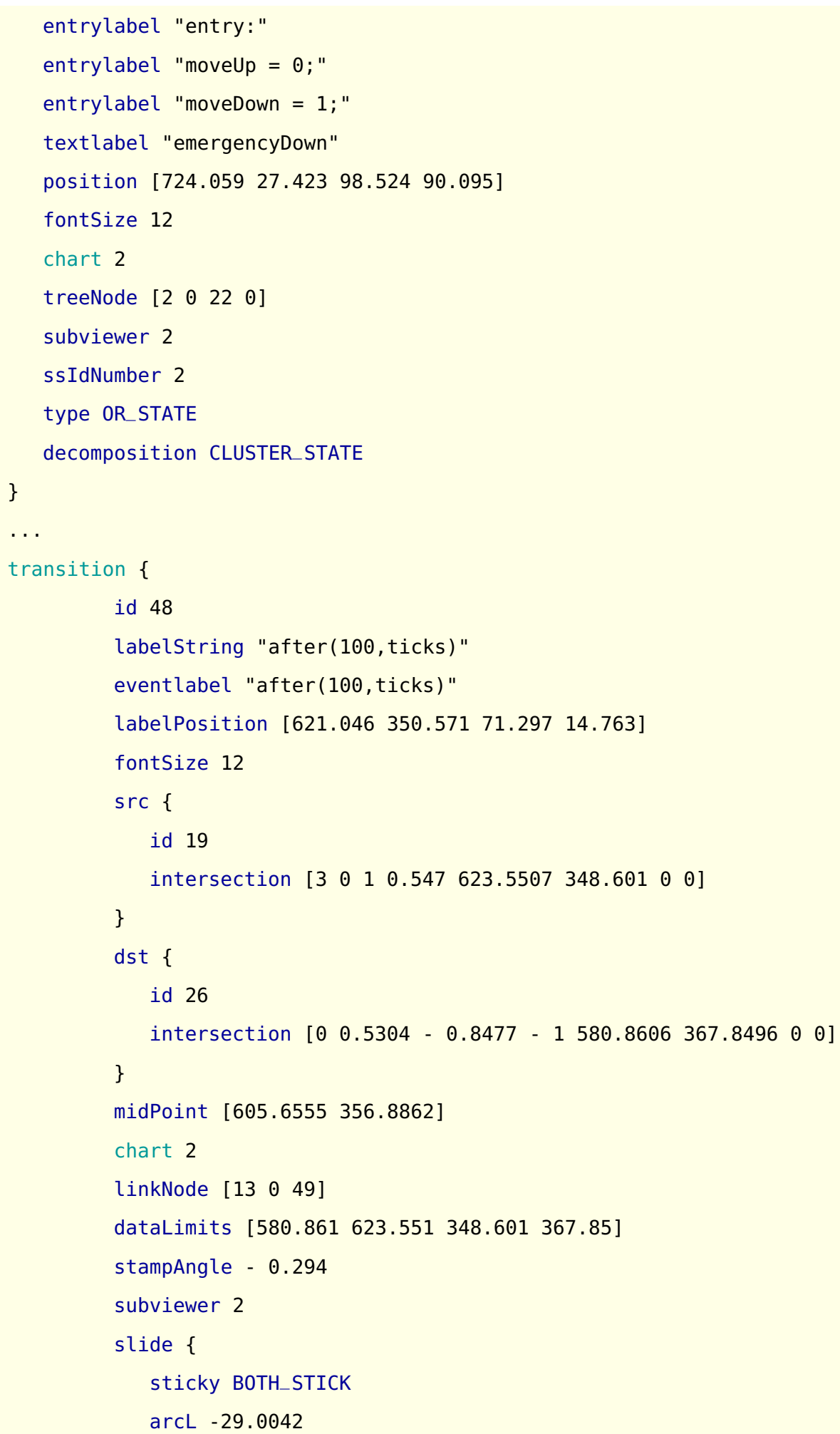




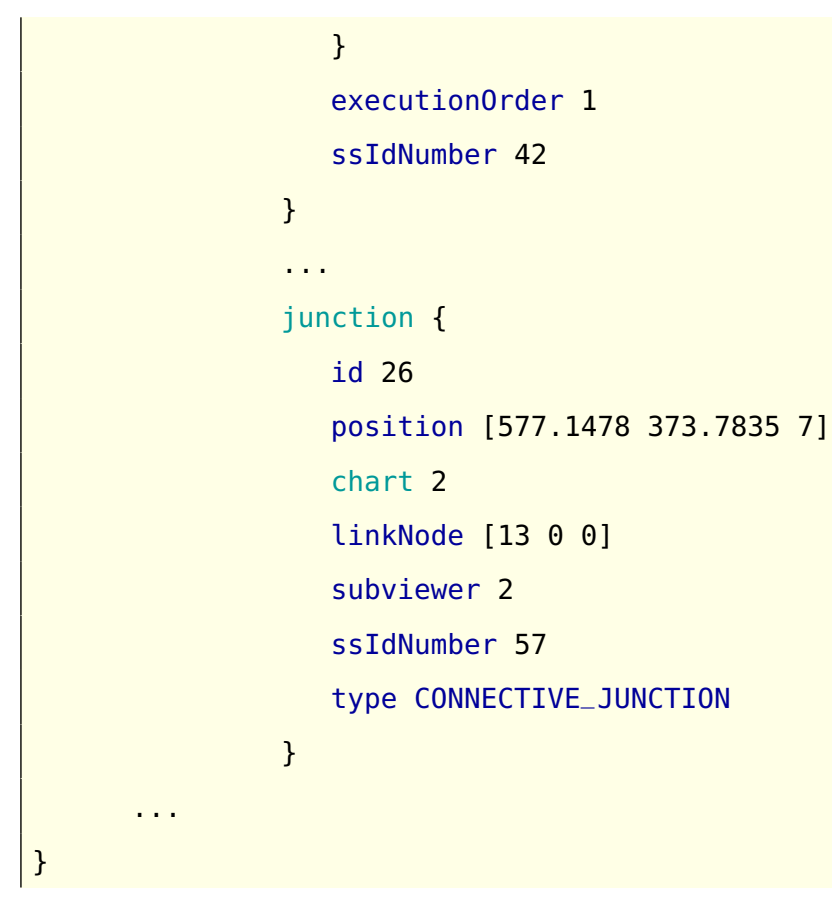

In order to avoid those irrelevant difference overwhelming the similarities in fragments of models, we designed a filtering plugin to identify and remove irrelevant elements from extracted fragment potential clones. Due to the lack of definitive documentation for the text form of Stateflow model files, we gradually tune our filters to remove irrelevant attributes as they are discovered. In the end, our filtering transformation removes ten elements in states level and seventeen elements in charts level to reduce the representation of model.

The similarity of some of the clone pairs identified by using only the extractor is increased when using these filters. For example, the similarity of "safe" state clone class has increased to $76 \%$. However, the filters do not identify more clone pairs and clone classes. The filtering can improve some similarity but not significantly. Figure 5.2 shows an example at chart level from two different Stateflow demo models, sldemo_auto_climatecontrol and sldemo_auto_climate_elec, which include the identical Temperature Control Chart. 

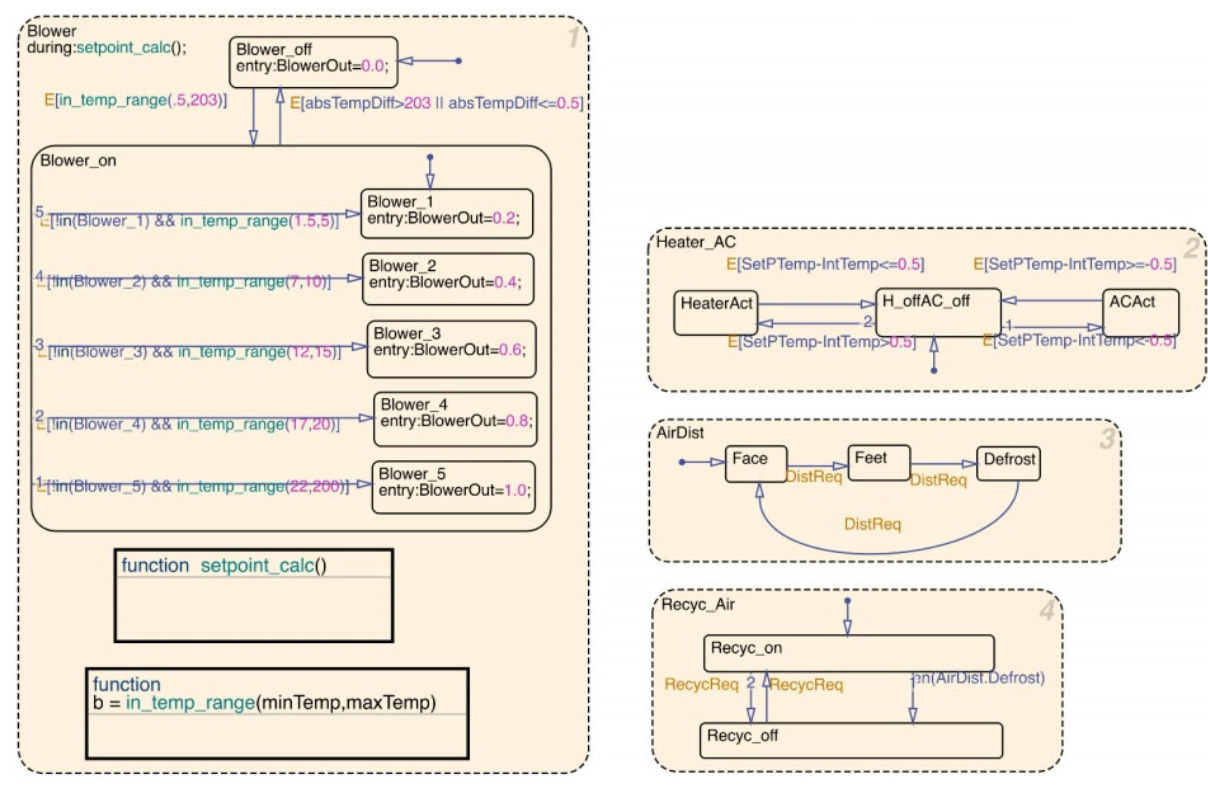

(a) ClimateControlSystem/Temperature Control Chart

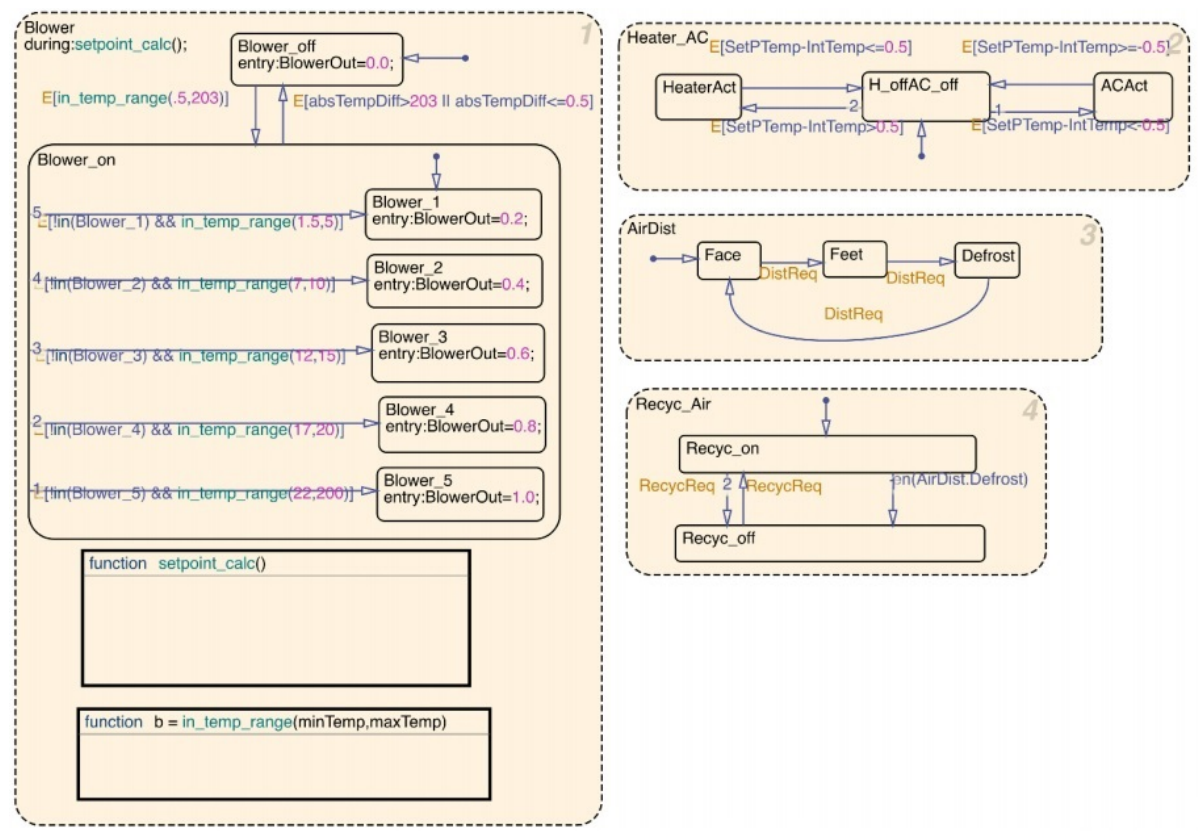

(b) Temperature Control Chart

Figure 5.2: Example of Stateflow clones from sldemo_auto_climate_elec.mdl and sldemo_ auto_climatecontrol.mdl in Matlab demo automotive models 


\subsubsection{Renaming}

As mentioned in Chapter 3, filtering improves the similarity of the clones, but SIMONE was not able to find all exact and near-miss state clones in the example model set. In order to identify the missing model clones, we also need to remove naming differences by adapting SIMONE blind renaming plugin for Stateflow.

We use a fixed value " $x$ " to rename all attributes that represent internal information, and they are not relevant to clone comparison. In SIMONE agile parsing is used during the parsing phase to grammatically distinguish the attributes that need to be renamed from those that should not be renamed.

Renaming significantly improved recall in finding exact and near-miss exact state and chart clones in the Stateflow demo models. Table 5.1, the Filtered \& Renamed column, shows the result of renaming. We found 281 state clone pairs clustered in 44 clone classes, and 728 chart clone pairs clustered in 27 clone classes. New type 2 and type 3 clones were identified and the following examples are some of these cases.

Figure 5.3 shows an example type 2 clone of four different states in one chart in the powerwindow model of the Simulink example set. As you can see from the figure, the structure of each state is exactly the same, but the names and labels have been changed, replacing the string "passengerDown" with the string "passengerUp", "driverDown" and "driverUp".

Figure 5.4 shows a type 3 clone between the function $e$ and function $r$ in Game chart of sf_tetris model of the Stateflow demo set. They have almost identical structure except that function $e$ has two more junctions and one more transition marked in red circle. The clone fragment of model is a function in this example. Figure 5.5 shows another type 3 


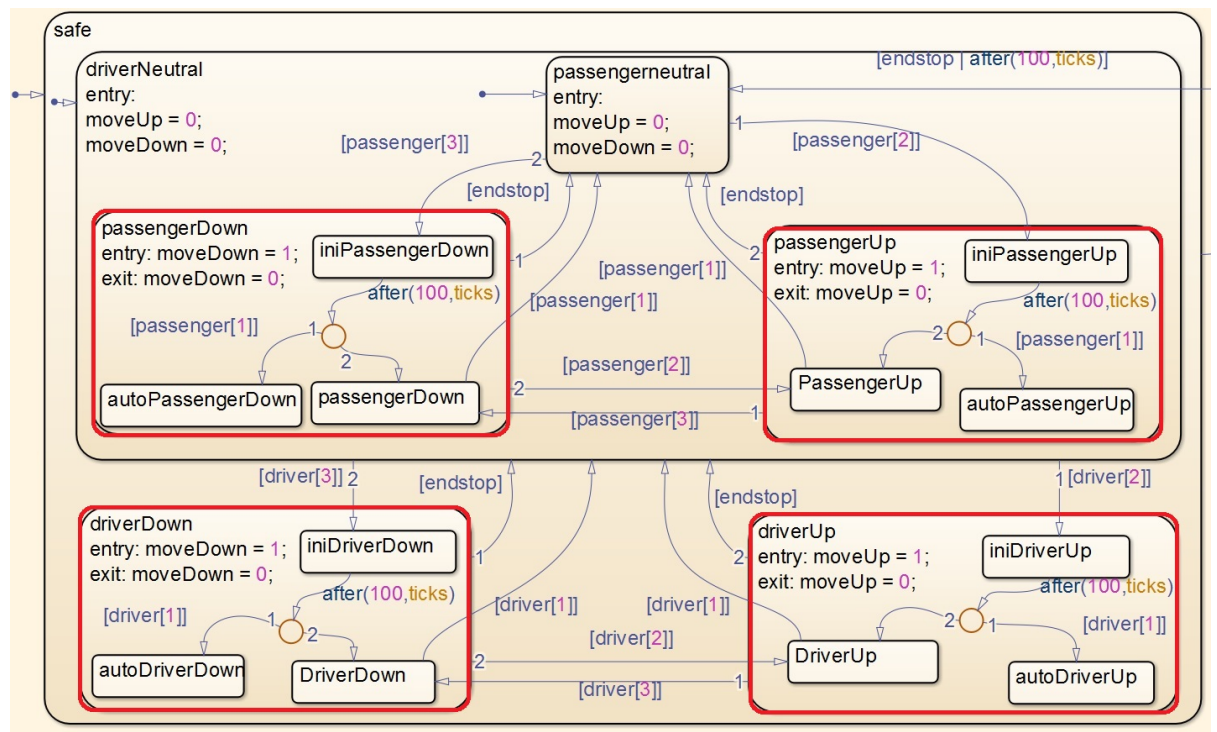

Figure 5.3: A Type 2(renamed model clone), this example is in the powerwindow model. The four red states are similar to each other. SIMONE similarity $76 \%$

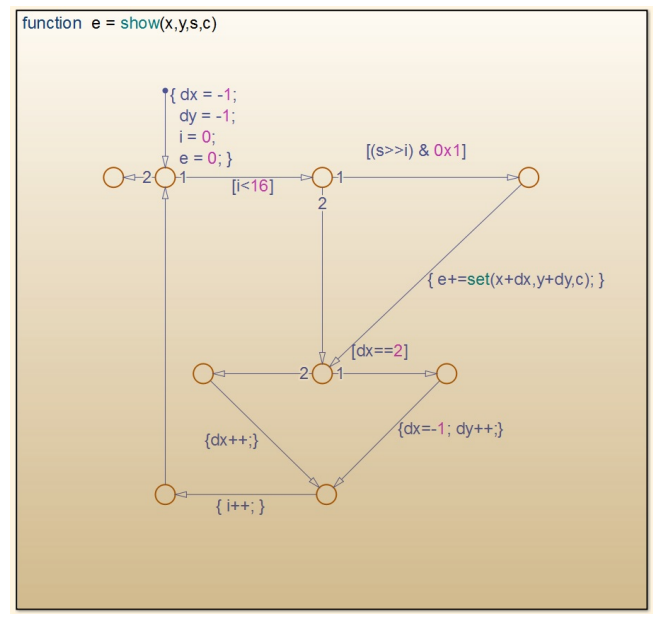

(a) sf_tetris/Game function e

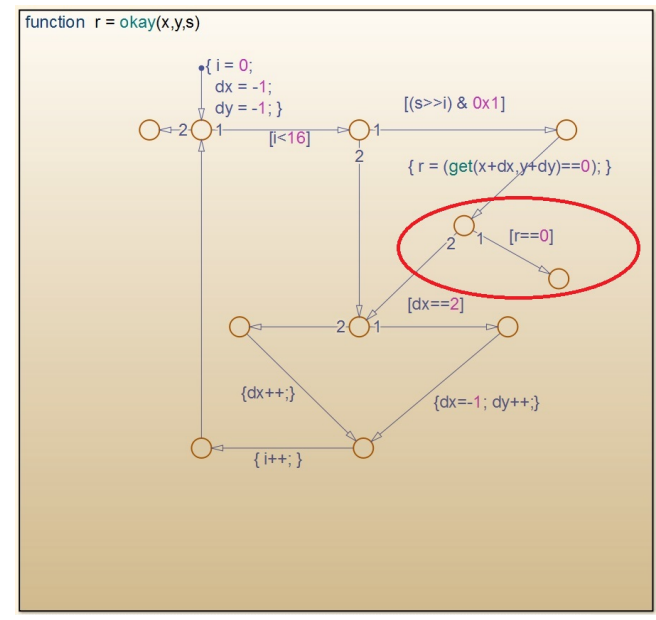

(b) sf_tetris/Game function $\mathrm{r}$

Figure 5.4: A Type 3(near-miss model clone), both function e and $r$ in the same chart Game in sf_tetris model. The function form the of fragment of clone. SIMONE similarity $71 \%$. 


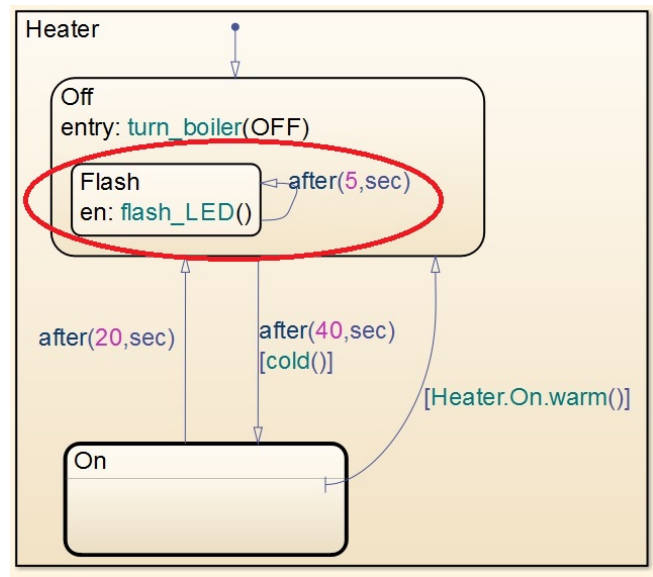

(a) sf_boiler/Bang-Bang Controller

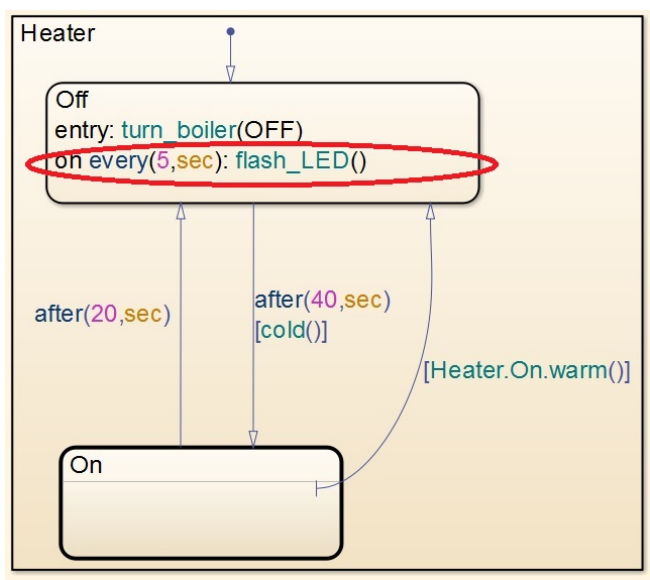

(b) sldemo_boiler/Bang-Bang Controller

Figure 5.5: A Type 3(near-miss model clone), (a) in sf_boiler model and (b) in sldemo_ boiler. SIMONE similarity $81 \%$.

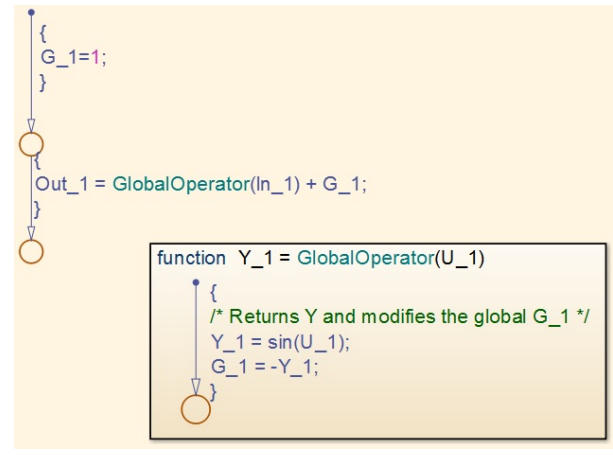

(a) ex_mg_hisl_0062/One

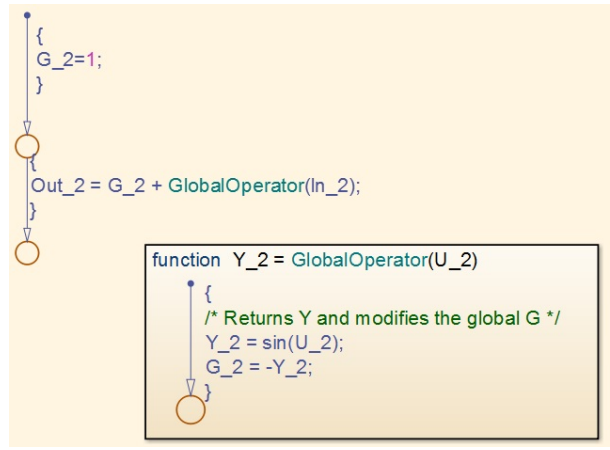

(b) ex_mg_hisl_0062/Two

Figure 5.6: A Type 2(renamed model clone) chart clone, both (a) and (b) in the ex_mg_ hisl_0062 model.

clone between Bang-Bang Controller/heater state of sf_boiler model and Bang-Bang Controller/Heater state of sldemo_boiler model of the Staeflow demo set. The red circles shows the difference between the two states.

Figure 5.6 shows an example type 2 clone of two different chart, One and Two, in the ex_mg_hisl_0062 model of the Simulink example set. As you can see from the figure, the 


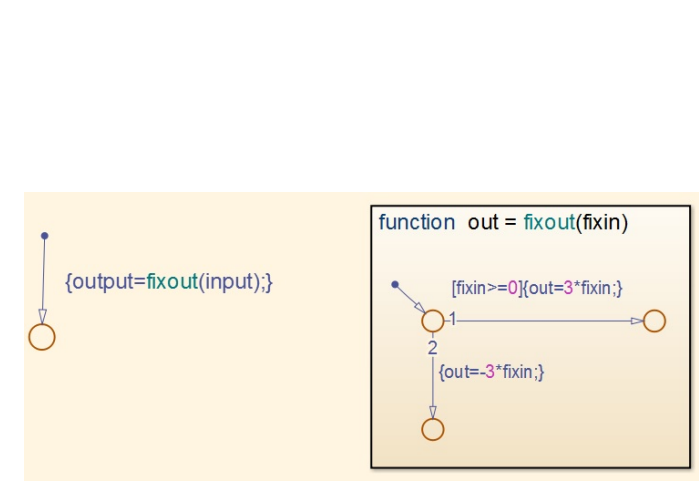

(a) Fixed-Point Graphical Functions/Chart

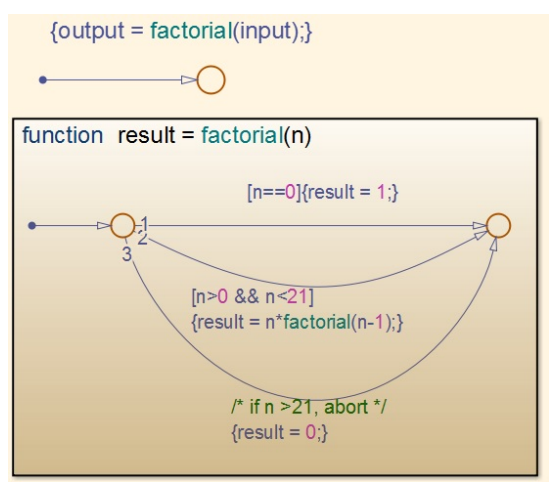

(b) Recursive Graphical Function Demonstration/Chart

Figure 5.7: A Type 3(near-miss model clone) chart clone, Fixed-Point Graphical Functions in sf_fxptgf model and Recursive Graphical Function Demonstration in sf_gfrecursive

structure is the same, but the labels have been changed, replacing string "_1" with string “_".

Figure 5.7 shows a type 3 clone between the Fixed-Point Graphical Functions Chart of the sf_fxptgf model and the Recursive Graphical Function Demonstration Chart of the sf_gfrecursive model of the Stateflow demo set. A new transition has been added and one junction has been removed, meanwhile naming and attribute has changed to other transition and lines.

\subsubsection{Sorting}

While renaming improves Stateflow clone detection, we found that the order of Staeteflow graphical objects in two identical models may be different from each other. SIMONE compares potential clones line by line. Thus the order of graphical objects in textual representation of a model does not change its graphical meaning but it will affect the identification of clones. We developed an sorting plugin in SIMONE for Stateflow model, which uses 
the size sorting criteria to sort the states by the number of nested elements.

Listing 5.2: Example snippet of sf_aircraft_screen_library

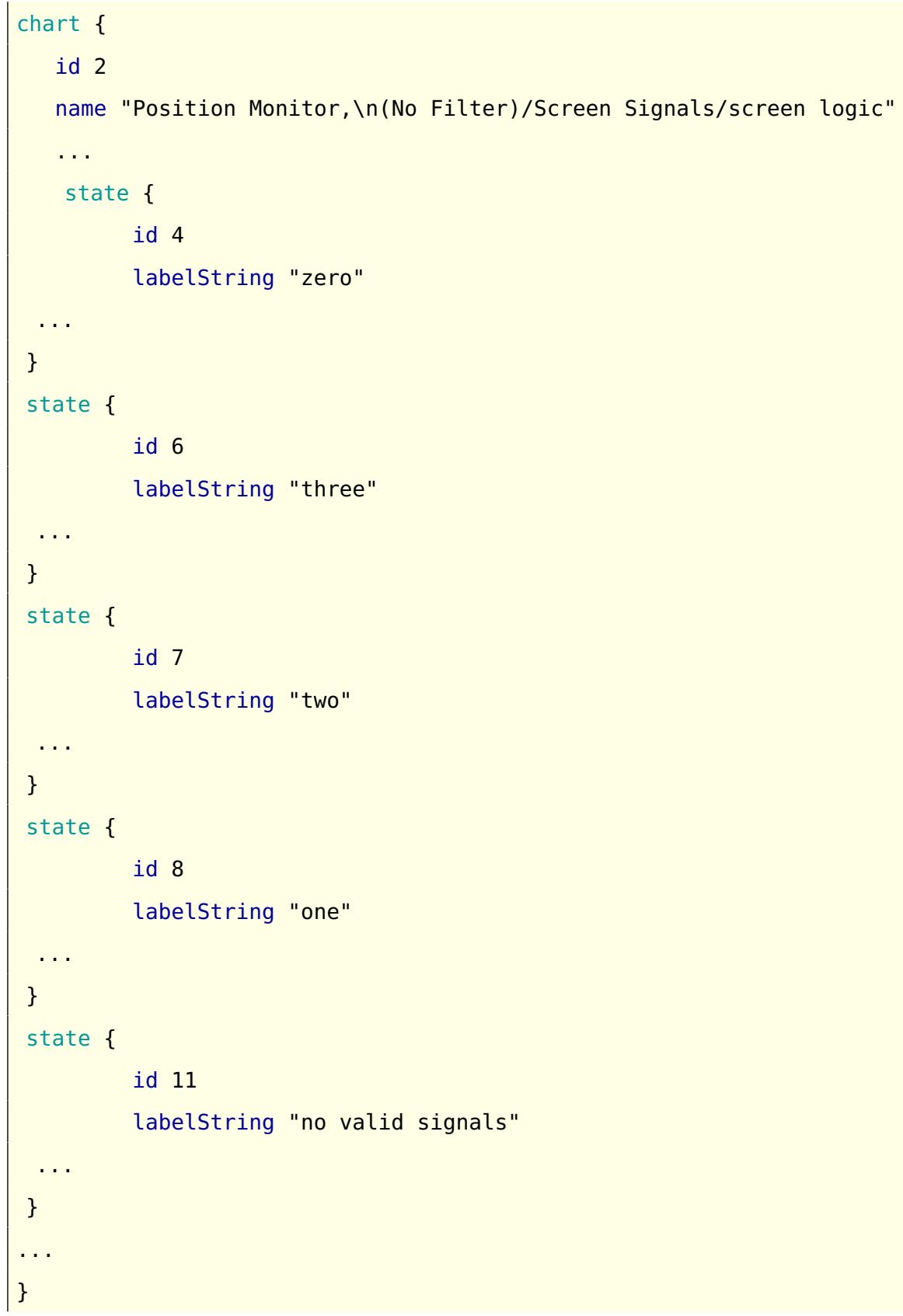


Listing 5.3: Example snippet of Hydraulic Monitor of sf_aircraft_screen_library.

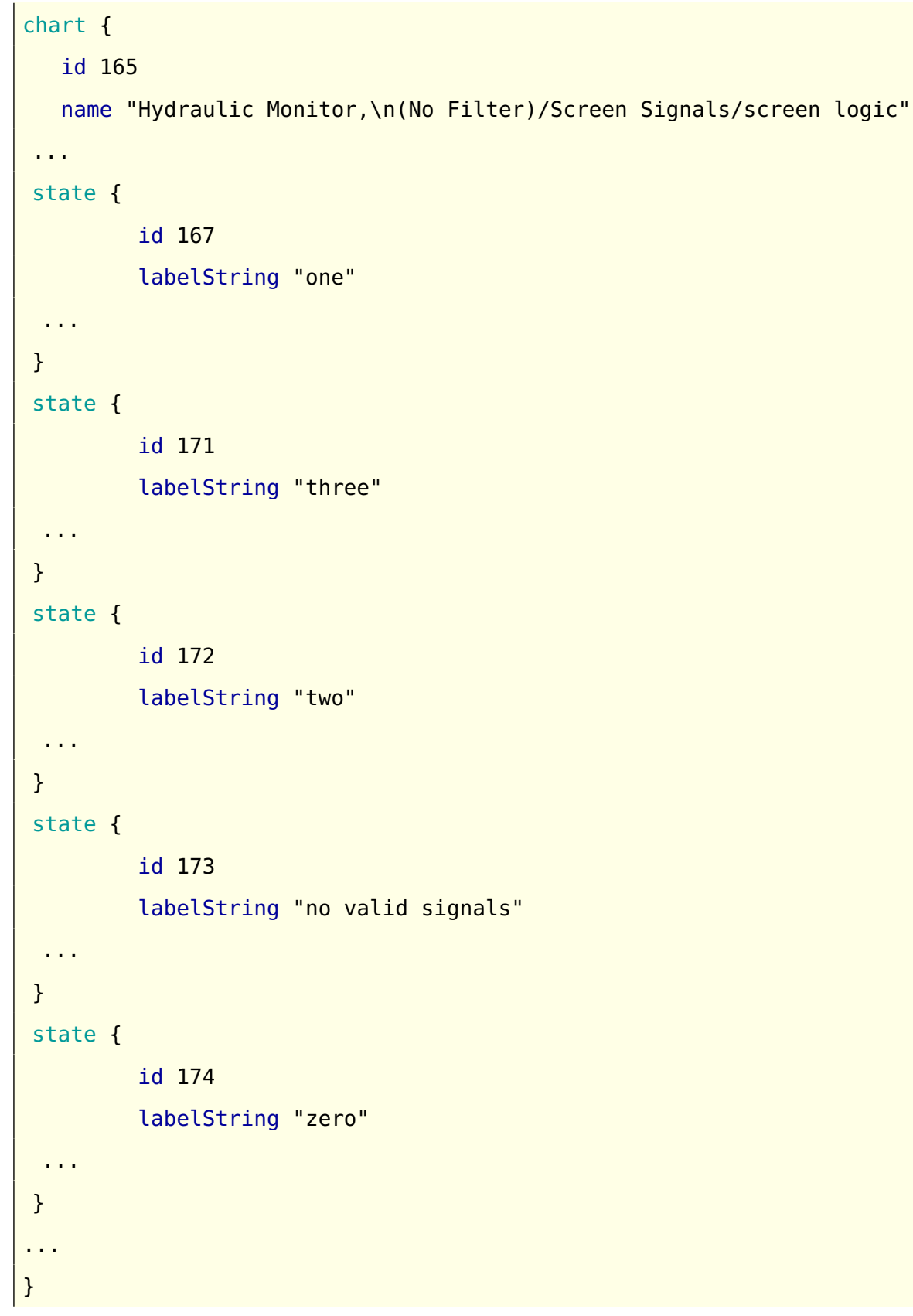

Listing 5.2 and listing 5.3 show the textual presentation of sf_aircraft_screen_library model in the Simulink demo set. They both have the identical states "zero", "one", "two", 
"three" and "no valid signals" and they have different order inside the model file. We sort them by the size of each state, and we have the new order "no valid signals", "one", "three", "two", and "zero".

Table 5.1 shows the total number of clone pairs and classes detected by each of these options. Filtering reduced the total number of clones by removing the false positives gen-

erated by similarities only in unimportant attributes. Renaming increased the number of clones detected by allowing different names to match. Sorting improved the quality resulting in slightly fewer clone pairs, but a few more clone classes.

\subsection{Summary}

In this chapter, we present the extraction and normalization phases in more detail. We discuss the extractor written for state and chart. The necessary normalization steps including filtering irrelevant elements, renaming the value of irrelevant attribute, and sorting the graphical object in textual representation. In the next chapter, we will consider the contextualization of Stateflow model. 


\section{Chapter 6}

\section{Contextualization}

In the previous chapters, we discussed how we extended SIMONE, a Simulink near-miss Subsystem clone detector, to be able to detect clones in Stateflow models as well. However, clones in Simulink models and Stateflow models are detected separately when we apply SIMONE to the Simulink models. In order to enhance our SIMONE accuracy of Simulink clones, we also investigate explicating the state machines into the parent Simulink model since a state machine is a chart block within the Simulink model. In this chapter, we will present the detail of the process and results. We call this process contextualization, a process of putting the Stateflow model in a context, particularly in a Simulink model, for the purpose of letting the clone detector analyze the big picture to improve the efficiency of SIMONE. Figure 6.1 shows the detailed process of the contextualization stage.

\subsection{Simulink \& Stateflow Environment}

Simulink and Stateflow provide a powerful environment for modelling, simulating and analyzing real time dynamic systems. Usually most of the real time systems are hybrid systems that perform both continuous and discrete behaviours. Simulink can model systems 


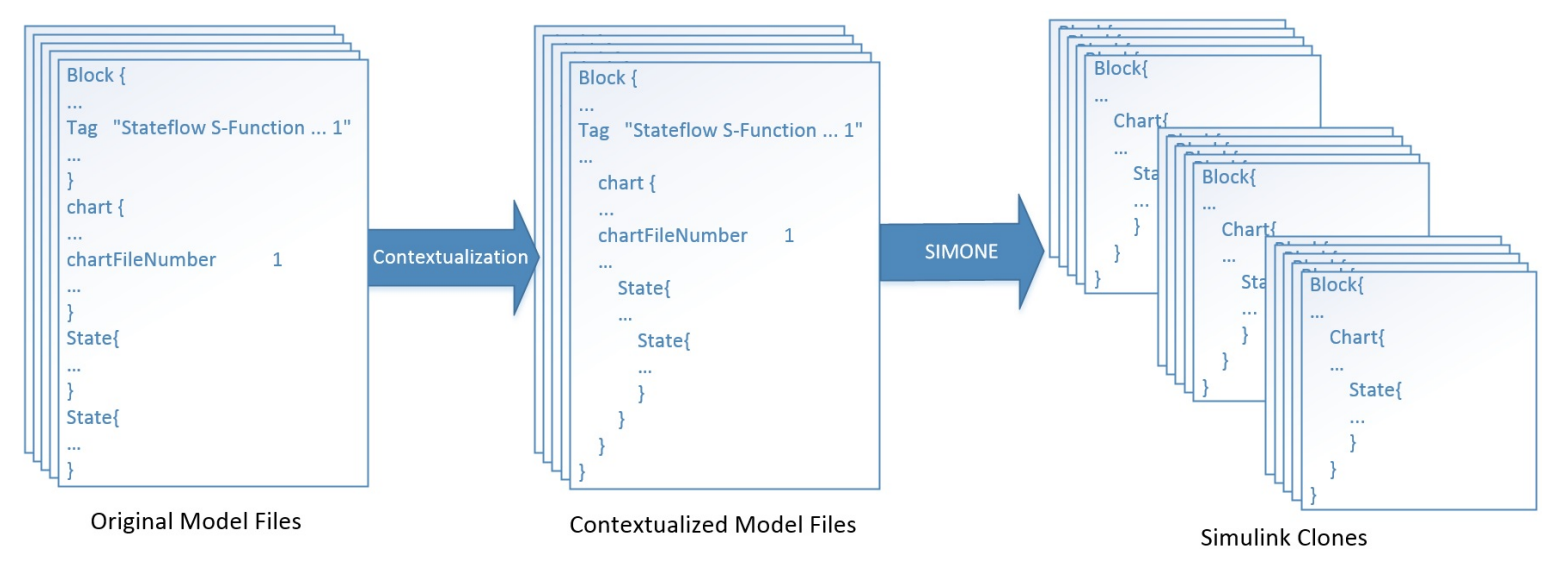

Figure 6.1: Contextualization: putting the Stateflow model in Simulink model for the purpose of letting the clone detector analyze the big picture to improve the efficiency

through building block diagrams in the GUI editor from a set of predefined blocks and associate these blocks by appropriate ports with the help of Stateflow, which functions as a block within the Simulink model.

\subsubsection{Graphic Representation}

Figure 6.2 shows an example of a system that contains both Simulink and Stateflow blocks. In the figure, the middle block named "control" is a Stateflow block and it works with other blocks and subsystems to form the "power_window_control_system" in the powerwindowlibsa.mdl Simulink model file. When a simulation is running, the execution of the model will automatically involve both the Simulink blocks and the Stateflow charts of the model.

We have seen how a Simulink model connects to a Stateflow model from the graphical representation in Figure 6.2. There are interfaces to enable Stateflow to communicate with Simulink models. The Stateflow interfaces are physical connections between Simlink and 


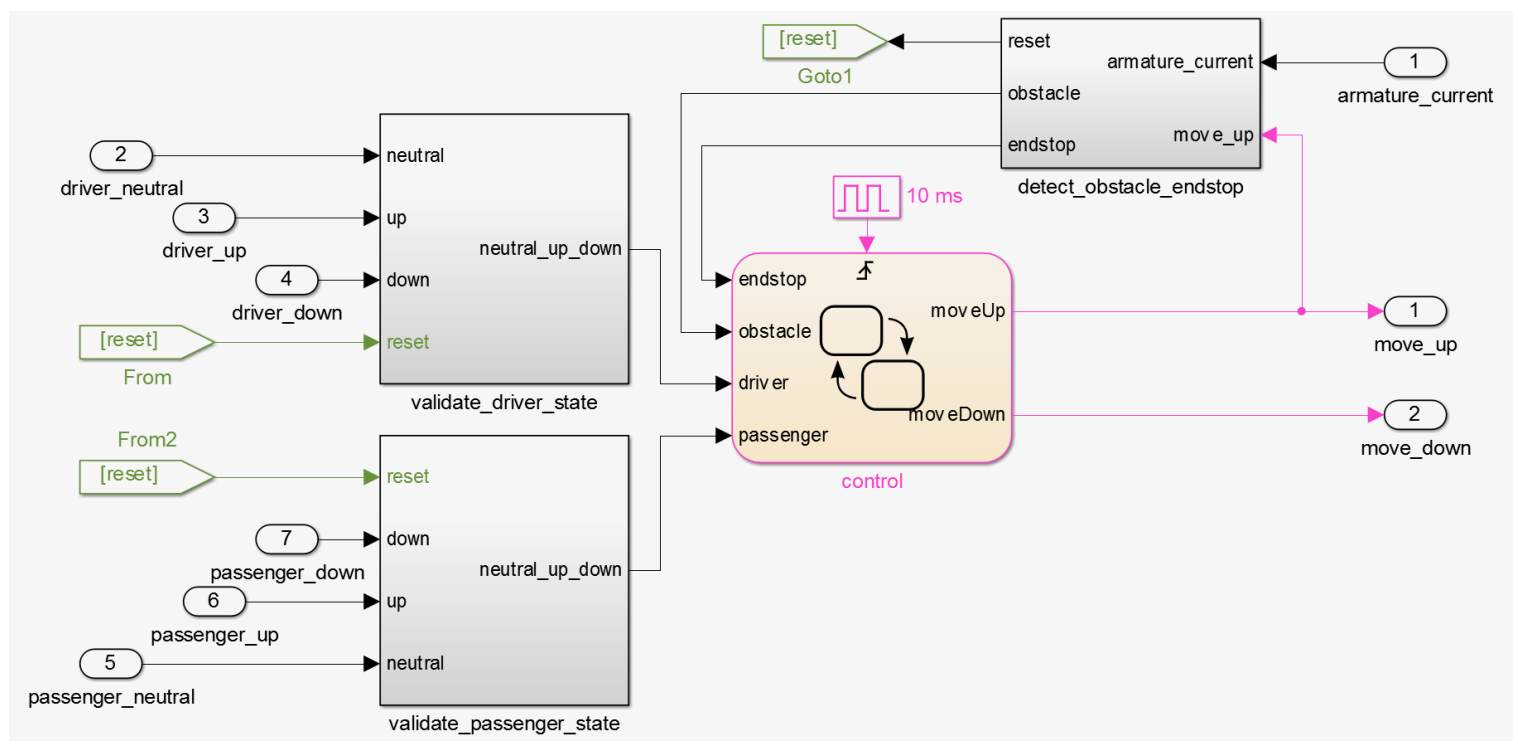

Figure 6.2: Graphical presentation of Simulink model with Stateflow: "power_window_ control_system" system consists of blocks, subsystems and "control" Stateflow block in powerwindowlibsa.mdl.

Stateflow blocks; they exchange event and data information via these interfaces. The interface can perform some or all of the following tasks[20]: specifying the update method for a Stateflow block in a Simulink model, defining the output to Simulink and input from Simulink data and events, adding and defining any nonlocal data and events the Stateflow diagram must interact with, and defining relationships with any external sources.

\subsubsection{Textual Representation}

There is an important element in the chart object textual representation functions as the interface, which identifies the connection between the Stateflow chart and its Simulink model. Listing 6.1 shows the important element chartFileNumber in the snippet. The value of chartFileNumber is an index[16], which indicates the order of the chart in the current file. The state chart block uses this number as a reference to its Simulink model. 
Listing 6.1: Example snippet of the extracted fragment used by Stateflow to store graphical models.

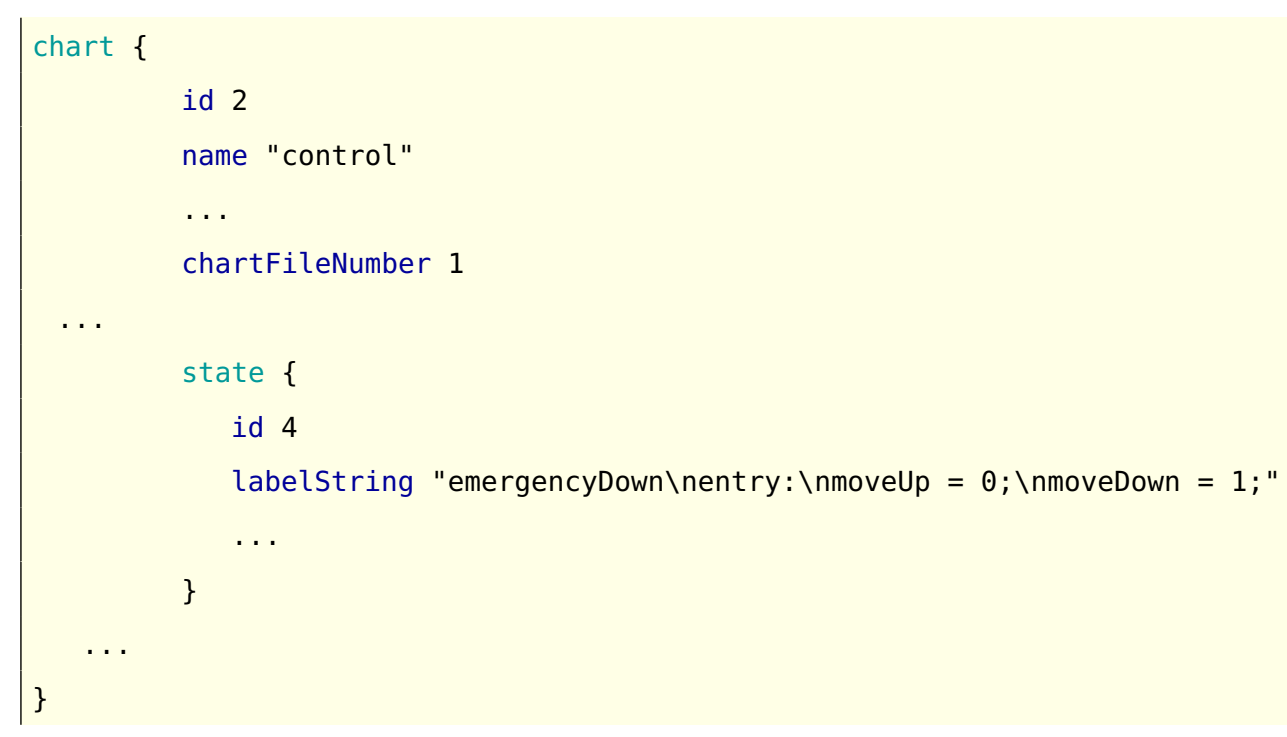

Accordingly, the Tag attribute element in Listing 6.2 is an element in the Simulink block textual representation to identify the connection to a Stateflow chart block. Simulink uses this Tag attribute to recognize a block referencing a chart, and a chart identifies itself as an S-Function block inside the Simulink model. The value of the Tag attribute has a certain format that starts with "Stateflow S-Function", followed by a model file name, and then the index number, like this: "Stateflow S-Function name number". The Tag value of the example in Listing 6.2 shows that it references a Stateflow chart which can be found in the model file powerwindowlibsa.mdl and has an index of 1 . If there is more than one chart in the model file, each chart has a unique sequential chart file number and a corresponding Simulink block with the same number at the end of the value. 
Listing 6.2: Example snippet of the extracted fragment used by Stateflow to store graphical models.

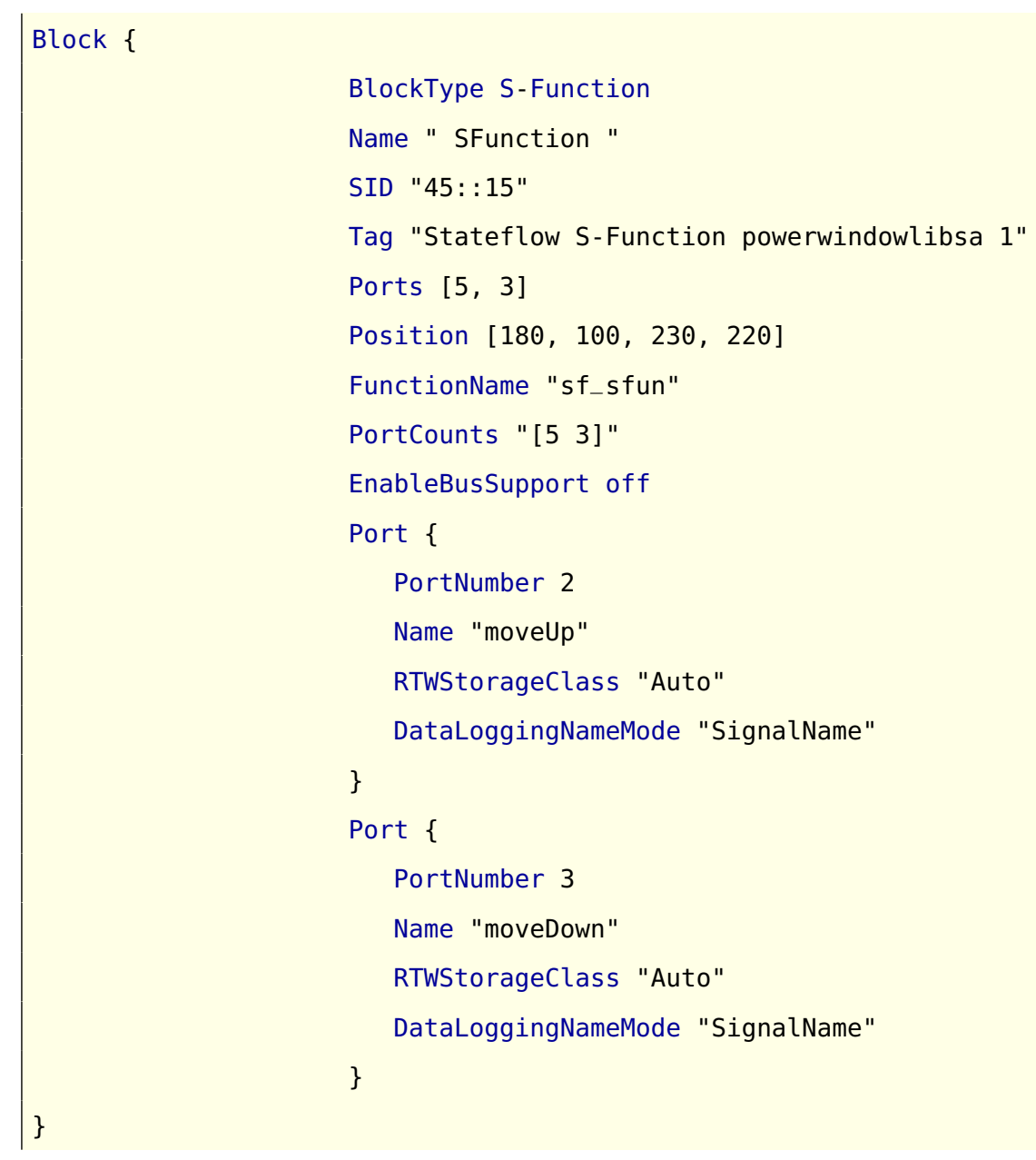

\subsection{States contextualization}

The purpose of the contextualization phase is to bring all the state charts referenced by the Simulink blocks into the self-contained unit in the textual representation level. We insert each chart into its parent block by the referencing number. Embedding the elements of each chart in this way creates a set of self-contained interaction units for comparison 
during clone detection. The contextualization is similar to the work done by Grant et al. [34] in identifying contextual clones in WSDL documents. In this thesis, we utilize this approach in three different ways: full lines, one line, and weighted lines.

\subsubsection{Contextualization via full lines}

Full lines means we put the entire state machine back to its parent Simulink. The chart text we used for full lines is the chart textual representation after normalization. Using full lines can increase the accuracy of SIMONE and avoid the noise from irrelevant elements, naming difference etc. There are several granularities in SIMONE for Simulink models. We chose the system level of granularity to detect potential clones for the contextualized fragments. Listing 6.3 shows the snippet of the contextualized textual representation of Figure 6.2. The System block has an "S-Function" block which contains the control chart. Listing 6.3: Example snippet of the contextualized fragment of "power_window_control_ system" system in in powerwindowlibsa.mdl.

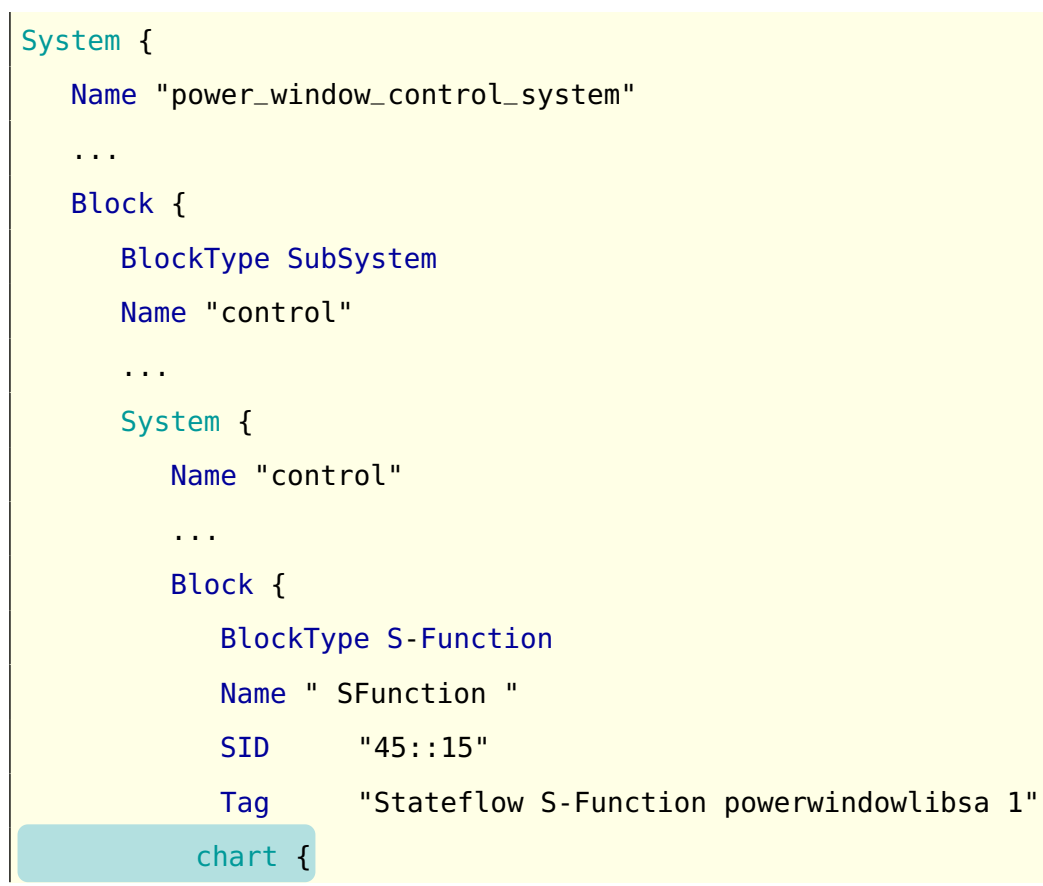




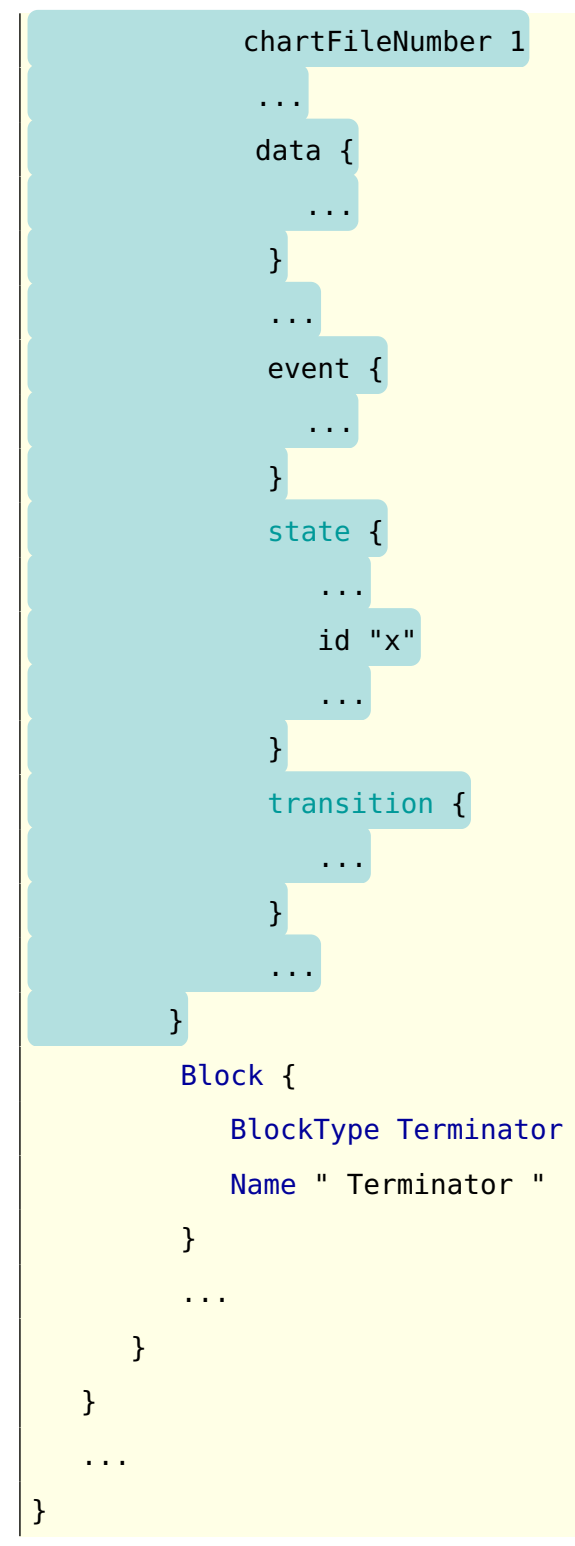

In our experiment, there were a few model files that contained Stateflow blocks but did not contain chart blocks in our sample set, so the model files we picked only contained both Stateflow and chart blocks that we could examine for the contextualization. The normalization plugins of SIMONE conformed with Stateflow, so we used filtering, blind renaming, and sorting plugins, and a threshold of $30 \%$ at the system level of granularity. We were expecting a more accurate result when using SIMONE on the combined models using the 
above parameter settings. Column two in table 6.1 shows the result of the full lines experiment. SIMONE can detect more clone pairs and clone classes after the contextualization.

\begin{tabular}{|c|c|c|c|c|}
\hline Total systems(1388) & System Only & Full lines & One line & Weighted lines \\
\hline Clone pairs & 4100 & 4902 & 4182 & 5058 \\
\hline Clone classes & 24 & 42 & 25 & 32 \\
\hline
\end{tabular}

Table 6.1: Initial results of the contextualization Stateflow model clones found in the Matlab demo set.

When examining the results, we can ignore all the systems without chart clones as they still remain the same as before, and mainly focus on those system clones containing charts. Changes in results can be classified into three categories:

1. System clones were still clones.

2. System clones were missing.

3. New System clones appeared.

SIMONE is a line-wised clone detector, so the number of lines of a chart embedded in a system potential clone candidate affects the result of the contextualized clone detection. If the number of lines in embedded blocks overwhelms the number of lines in the original Simulink hosting subsystem, then the charts' similarity will dominate the clone detection result. In our test set, the average number of lines is a system was 216 and the average number of lines is a chart is 342 . We can see the effect from the following three categories.

Category one, system clones had the same or similar charts in the cloned Simulink models. In our test set, there were several versions of the "powerwindow" model with a similar system "power_window_control_system" as shown in Figure 6.2, and all of them contained an identical chart "control" as shown in Figure 6.3. SIMONE can identify the 


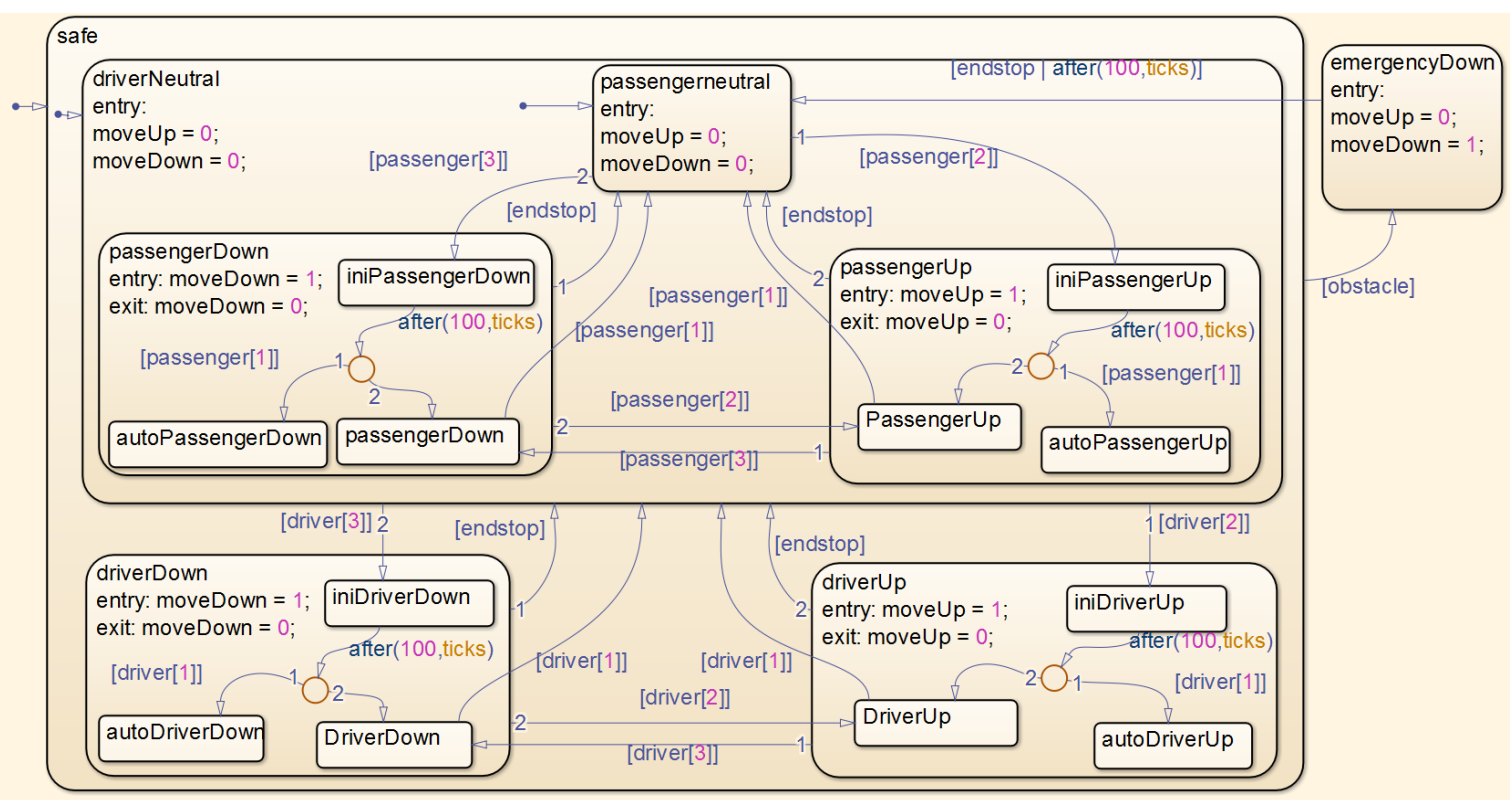

Figure 6.3: Graphical Representation of powerwindow model control chart ${ }^{1}$

"power_window_control_system" system clones both before and after contextualization as the identical chart.

Category two, system clones contained different charts and the size of the chart overwhelmed the hosting Simulink subsystem. For example, SIMONE reported that system "fp_verify_current/detect_obstacle" in powerwindowlibsa.mdl, and system "Mixing \& Combustion" in sldemo_fuelsys.mdl are cloned at 82\% similarity, shown in Figure 6.4. However, they have different chart blocks: chart "delay_detection" and function "EGO Sensor". "EGO Sensor" is a Matlab function block, which is internally represented as a chart block. Figure 6.5 shows the differences between the two charts embedded in the Simulink models. The size of both systems is 162 lines; the size of the "delay_detection" chart is 175 lines and the size of the "EGO Sensor" chart is 89. Obviously, the result of Simulink depends on the similarity of charts which dominate the similarity of the Simulink model.

\footnotetext{
${ }^{1}$ Power Window model is from MATLAB demo set.
} 


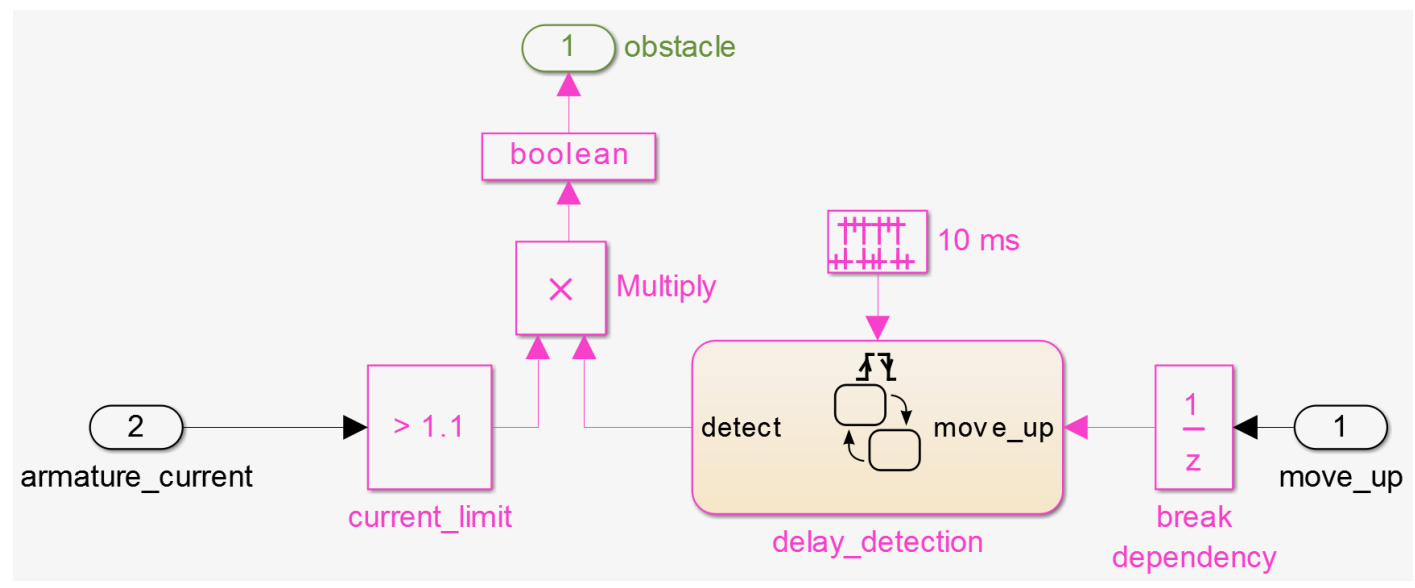

(a) System "fp_verify_current/detect_obstacle" in powerwindowlibsa.mdl

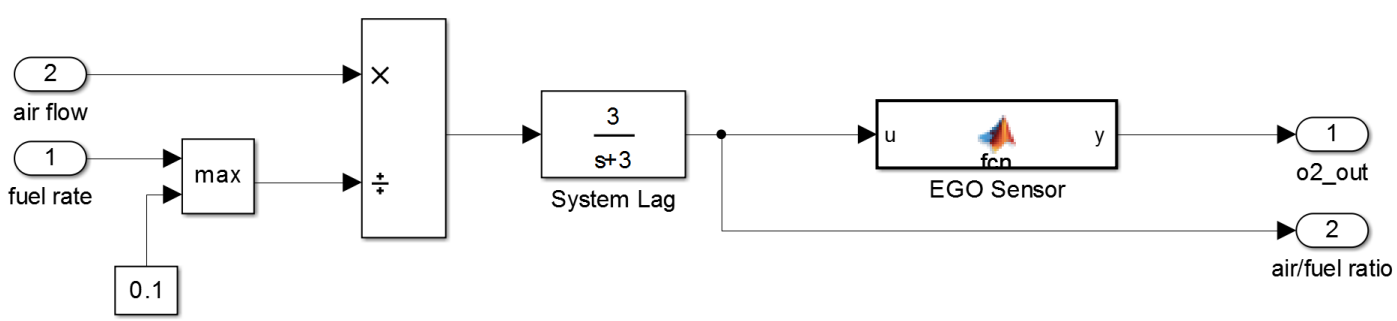

(b) System "Mixing \& Combustion" in sldemo_fuelsys.mdl

Figure 6.4: SIMONE can detect system "fp_verify_currentdetect_obstacle" and "Mixing \& Combustion" was a clone pair and the similarity of this clone pair was $82 \%$ before contextualization.

Figures 6.6 shows another example of a cloned model which disappeared from clone results of model file powerwindowlibsa.mdl, and that after the contextualization because of the same chart size overwhelming the subsystem size. System "fp_verify_current/detect_obstacle" has a chart "delay_detection", and system "power_window_control_system/ detect_obstacle_endstop/detect_obstacle" does not.

Category three, was the detection of the new cloned pair. Original non-cloned Simulink models contained the same or similar charts, and the size of the chart was big enough to lead to the clone detection result. SIMONE could not detect "powerwindow" system shown in figure 6.7(a) and "power_window_control" system shown in fighre 6.7(b) as a clone pair 


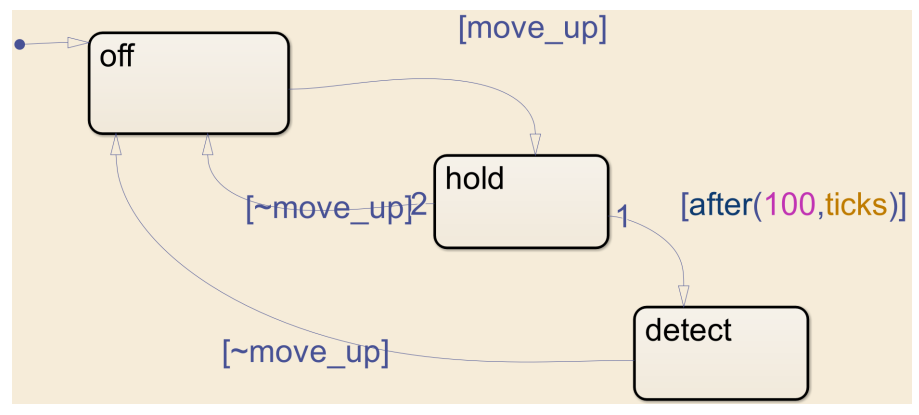

(a) Chart "delay_detection" in system "fp_verify_current/detect_obstacle"

• $\{$ eML_blk_kernel();\}

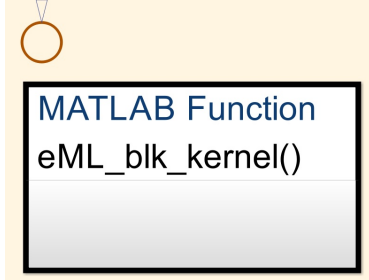

(b) The graphical representation of function "EGO Sensor" in system "Mixing $\&$ Combustion"

Figure 6.5: The different charts in system "fp_verify_currentdetect_obstacle" and "Mixing \& Combustion".

before contextualization. However, SIMONE could identify them at $82 \%$ similarity after the chart was embedded because both of them contained the same "control" chart, shown in Figure 6.3. SIMONE reported this clone pair based on a comparison of 1214 lines, and the "control" chart took about 810 lines. So the similarity of the chart contributed to this clone detection result.

\subsubsection{Contextualization via one line}

One line means we only put a reference line of Stateflow to its parent. As we can see from the above result in category one, if the size of a chart dominates the textual representation 


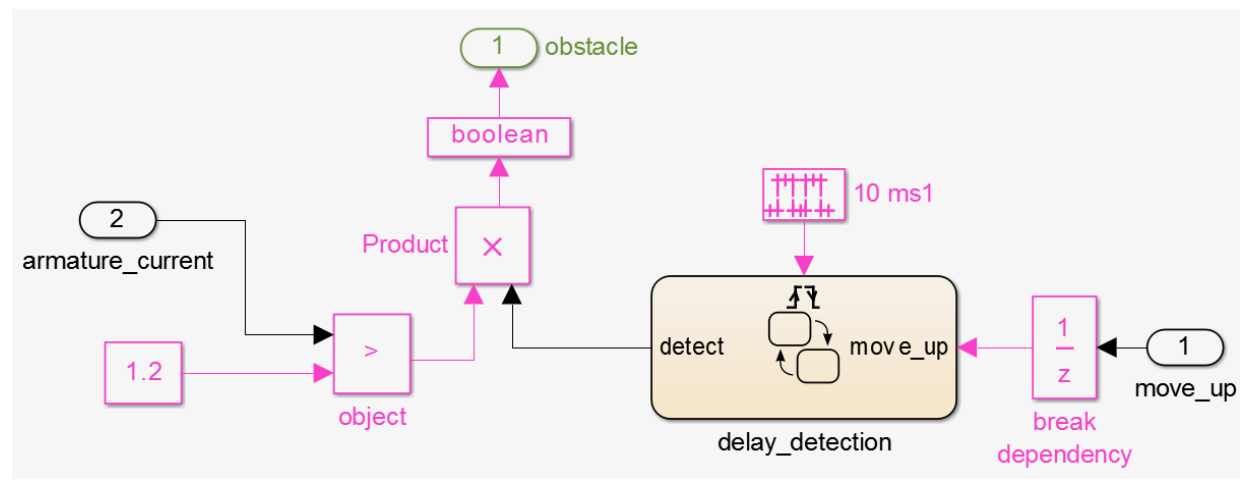

(a) System "fp_verify_current/detect_obstacle" in powerwindowlibsa.mdl

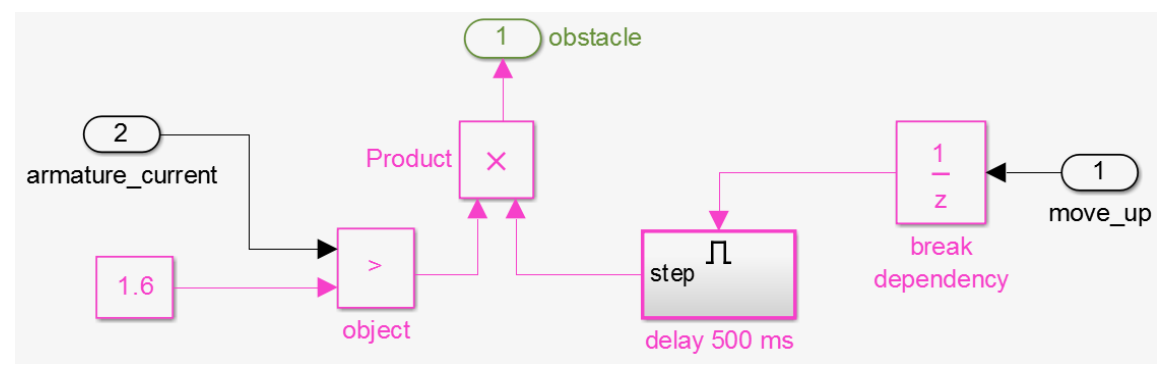

(b) System "power_window_control_system/detect_obstacle_endstop/detect_obstacle" in powerwindowlibsa.mdl

Figure 6.6: SIMONE can detect system "fp_verify_currentdetect_obstacle" and "power_ window_control_system/detect_obstacle_endstop/detect_obstacle" was a clone pair and the similarity of this clone pair was $71 \%$ before contextualization.

of a model, it will affect the Simulink model clone detection result. The full lines contextualization method is too aggressive. Thus, we come up with a single reference line idea. When we did the clone detection at a the granularity of chart level, SIMONE reported clone classes clustering similar charts into one group forming a class and assigning a unique number classid for this group. We embedded either this classid into its parent Simulink block or a unique number for those charts that did not belong to any class by using the same contextualization method. By doing this, we not only embedded the chart information in the Simulink model but also kept the similarity and differential of charts. 


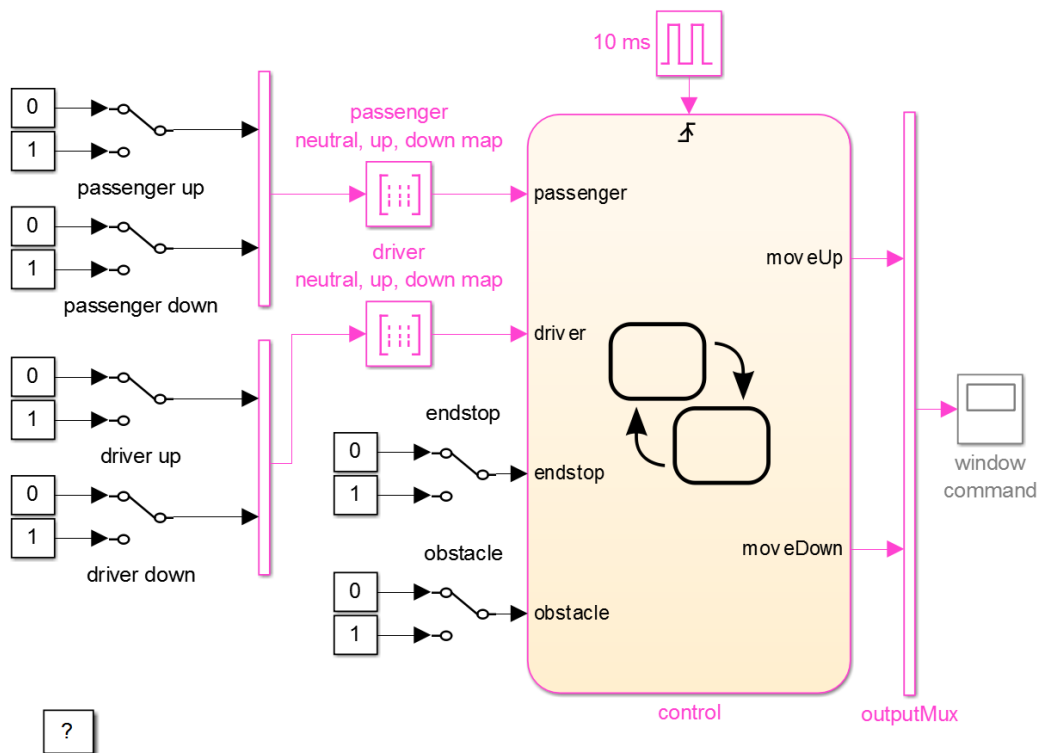

(a) System "powerwindow" in powerwindow.mdl.

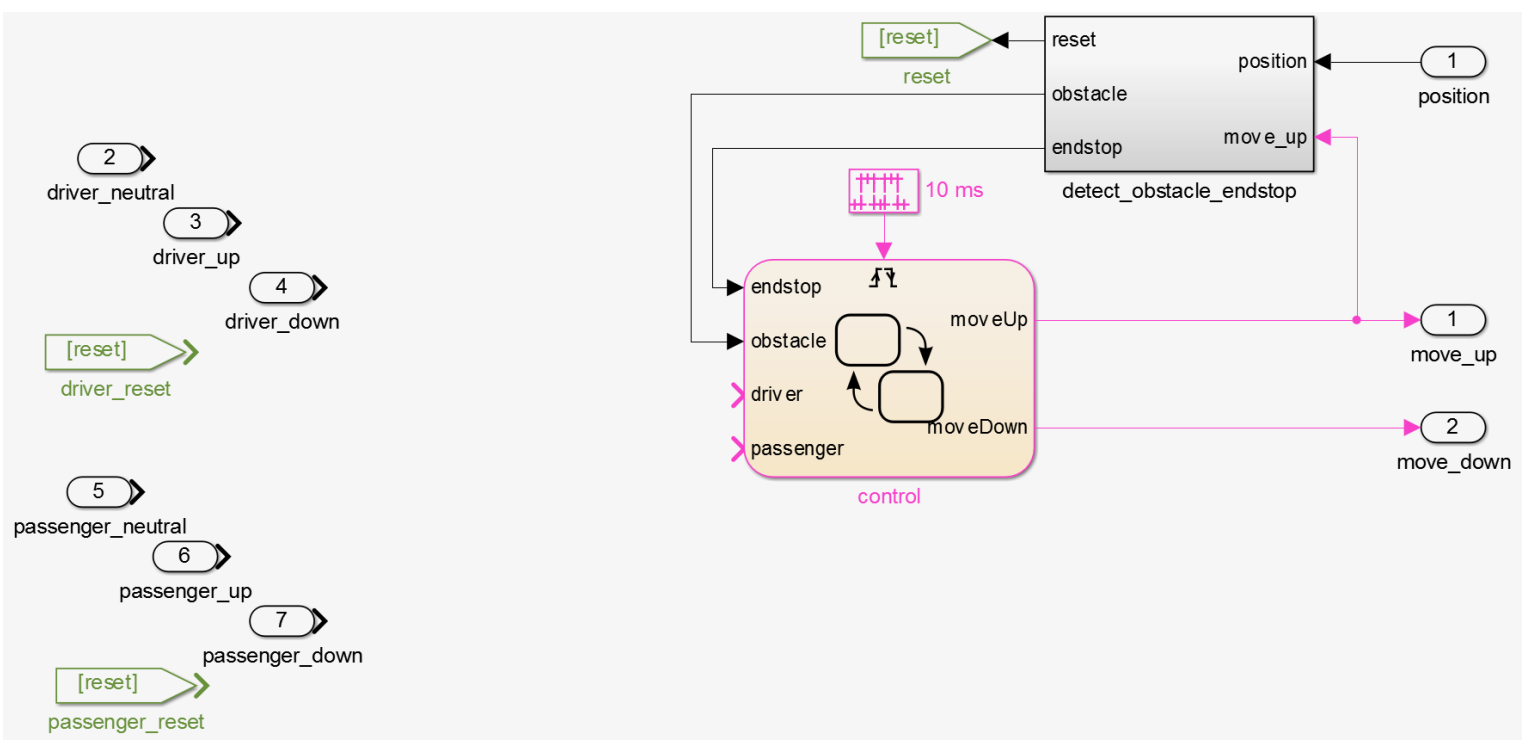

(b) System "power_window_control" in powerwindowlibsa.mdl.

Figure 6.7: SIMONE can detect system "powerwindow" and "power_window_control" was a clone pair and the similarity of this clone pair was $82 \%$ after contextualization as they contain the same chart. 
Listing 6.4 is an embedded one line version of the previous example in figure 6.2. Column three in table 6.1 shows the result of the one line experiment.

Listing 6.4: Example snippet of the one line contextualized fragment of "power_window_ control_system" system in in powerwindowlibsa.mdl.

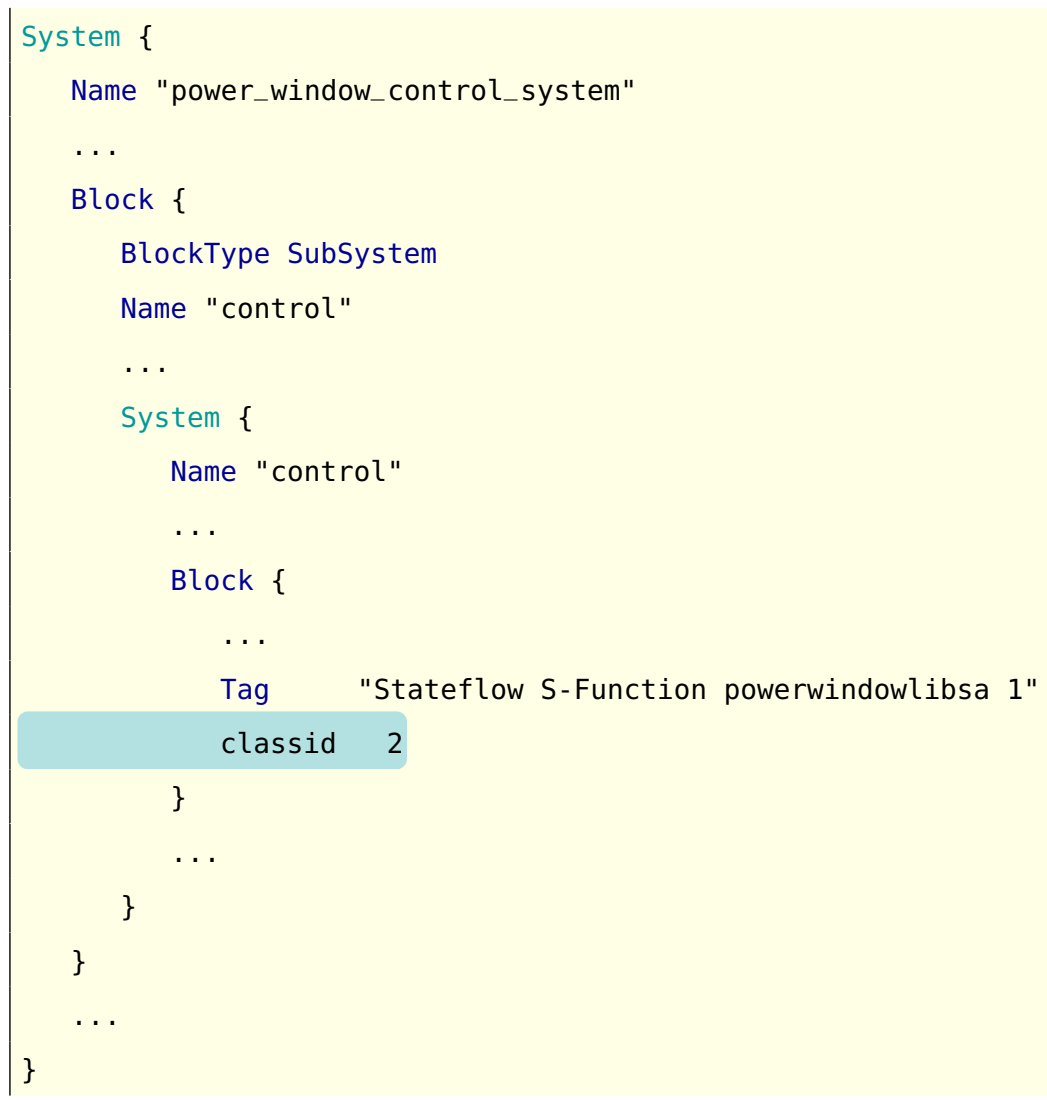

The one line contextualization clone detection result still falls in the same three categories. The clone pairs in category one, system clones were still cones, take the majority of the result. There are 4038 clone pairs belonging to category one in 4100 total clone pairs and most of them have the same similarity percentage as before, some of them are within $\pm 1 \%$ range. Figure 6.8 and Figure 6.9 show an example of category two, system clones were missing; they are a clone pair with $71 \%$ similarity before contextualization. Both of these two models contain Embedded Matlab Function, which is internally represented as a 
chart that contains a single state and an initial transition in the textual representation. After contextualization, the clone pair did not show up in the clone report and the similarity of this pair is $70 \%$. We examined all of the 62 missing clones pairs, all of them are $71 \%$ similar. Figure 6.10 and Figure 6.11 show an example of category three, new system clones appeared, this is a new clone pair and the similarity is $71 \%$. So the one line changes have a marginal effect on the contextualization.

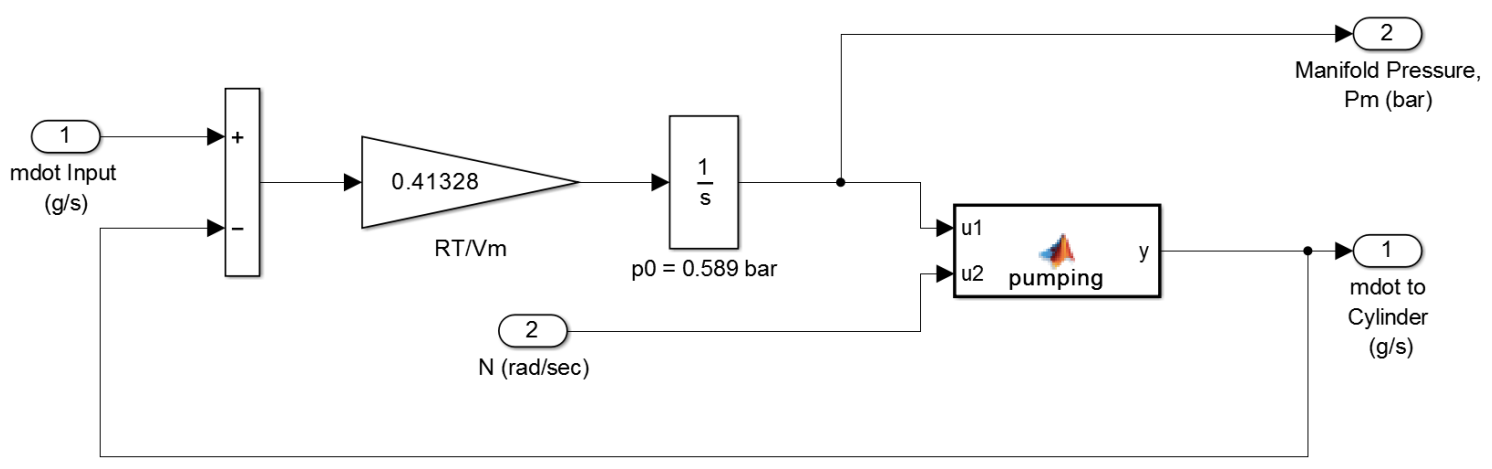

Figure 6.8: System “Intake Manifold" in sldemo_fuelsys.mdl.

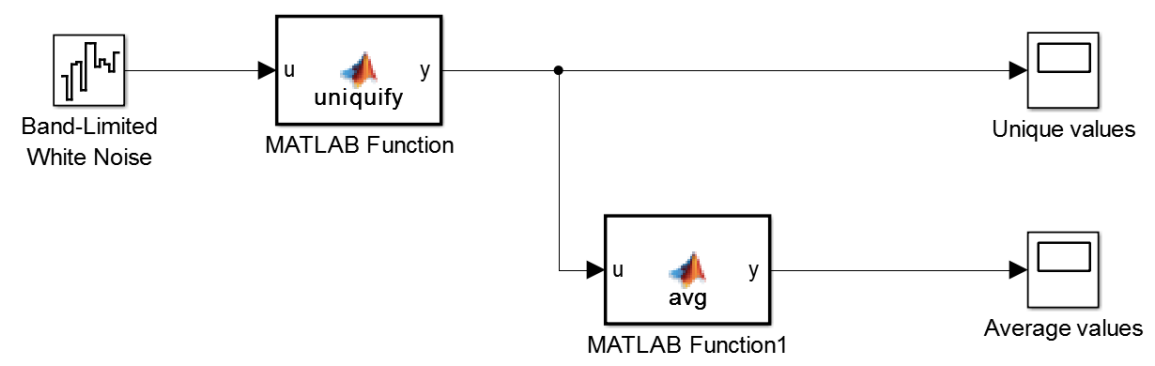

Figure 6.9: System “emldemo_process_signal" in emldemo_process_signal.mdl.

\subsubsection{Contextualization via weighted lines}

Weighted lines means putting an average weight of a Stateflow back to its parent. Full line (i.e., entire chart ) is aggressive and one line is marginal. Thus, we did another experiment 


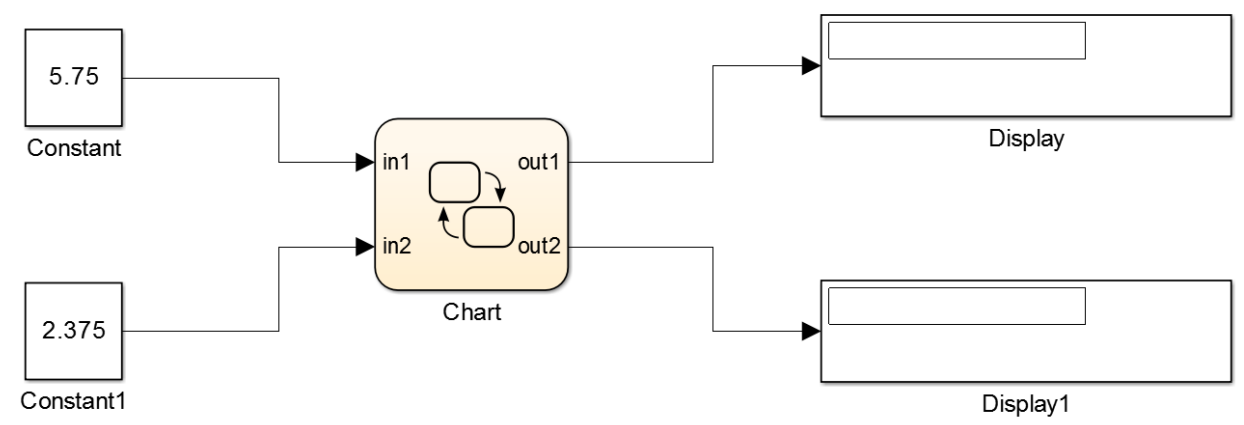

Figure 6.10: System "sf_fxptprecision", which is a Fixed-Point multiplication using Stateflow, in sf_fxptprecision.mdl.

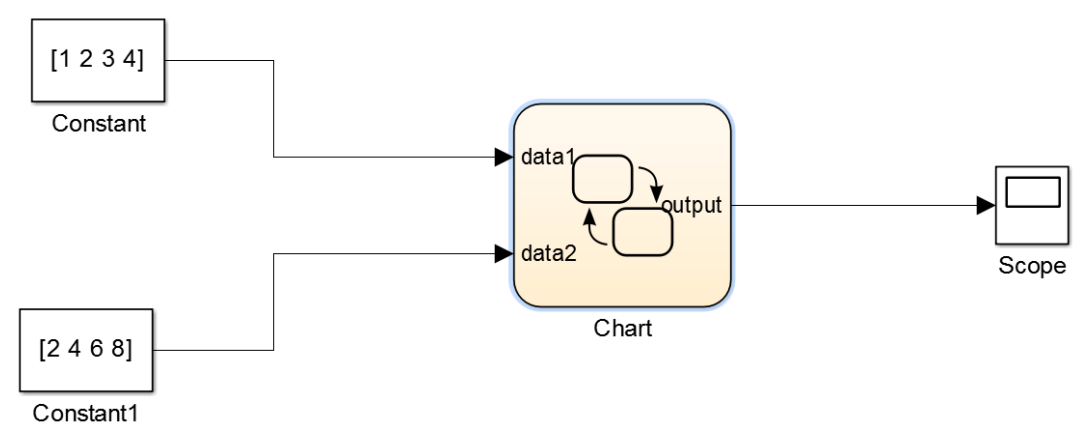

Figure 6.11: System "sf_array", which is a Stateflow vectorization, in sf_array.mdl.

called weighted line that gives each chart a certain weight(line) and then embed the certain lines to present a chart in the Simulink model. Based on our group experience, the average lines of a block are ten, so we give each chart a weight at ten lines. We multiply the single line from one line experiment by ten to get the weighted chart, then we embed it into its parent Simulink block. Listing 6.5 is an embedded weighted lines version of the previous example in figure 6.2. Column three in table 6.1 shows the result of the weighted lines experiment. 
Listing 6.5: Example snippet of the weighted lines contextualized fragment of "power_ window_control_system" system in in powerwindowlibsa.mdl.

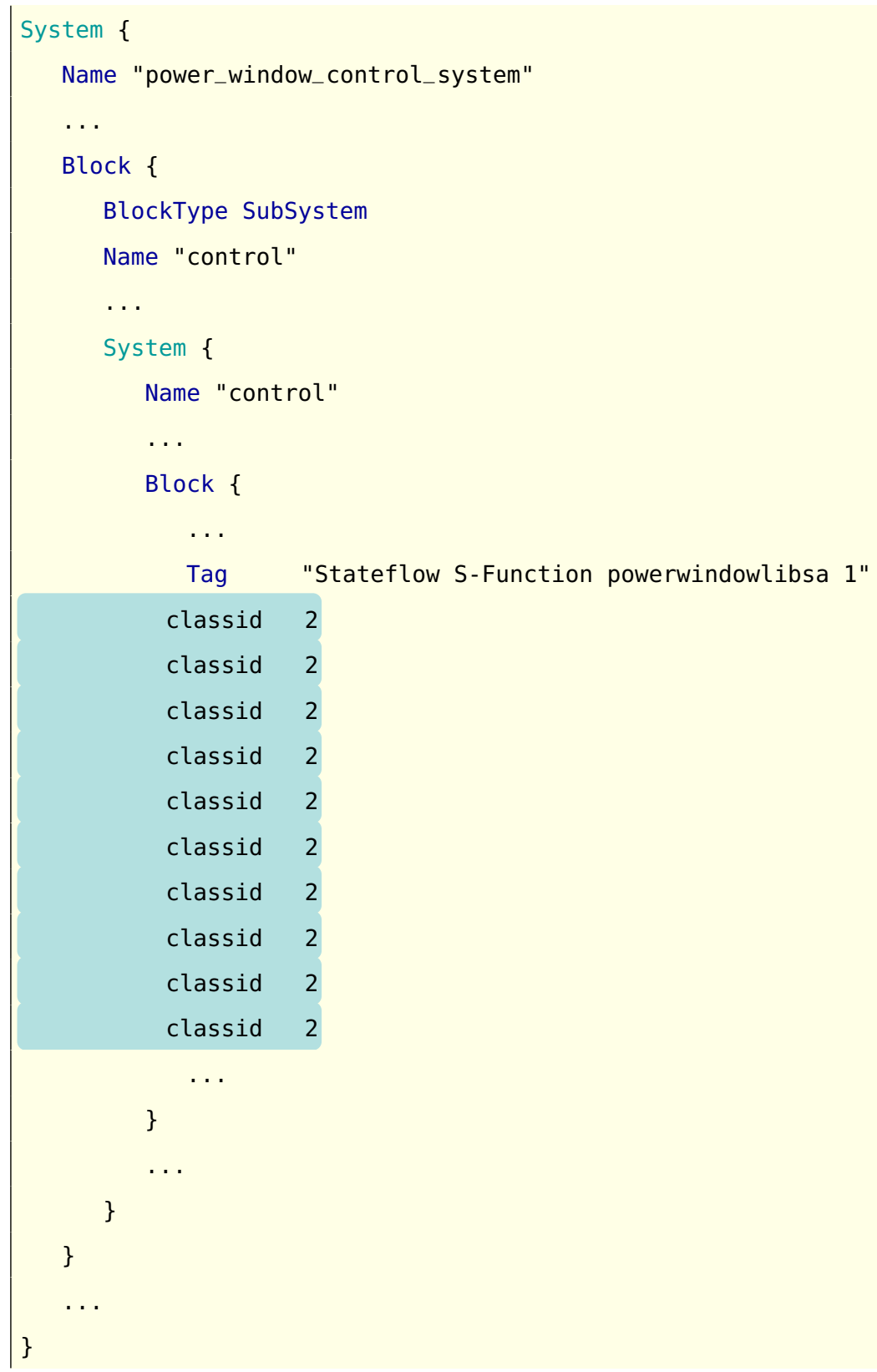

The result of weighted lines is similar to the previous one line experiment. There are 3603 clone pairs belonging to category one and still taking the majority part of the result. 


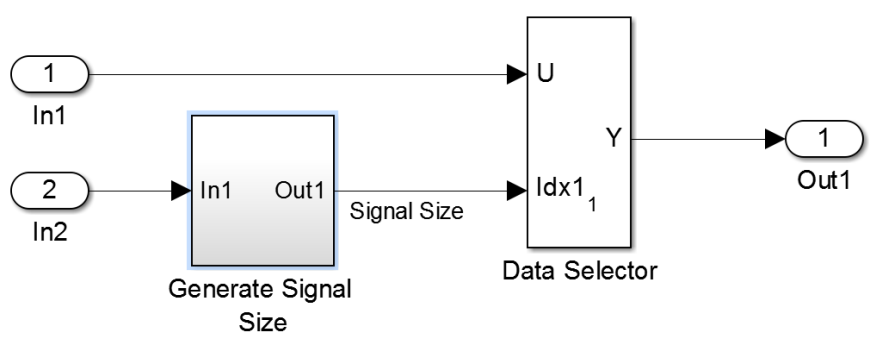

(a) System "Length Adaptation" in sldemo_varsize_dataLengthAdapt.mdl.

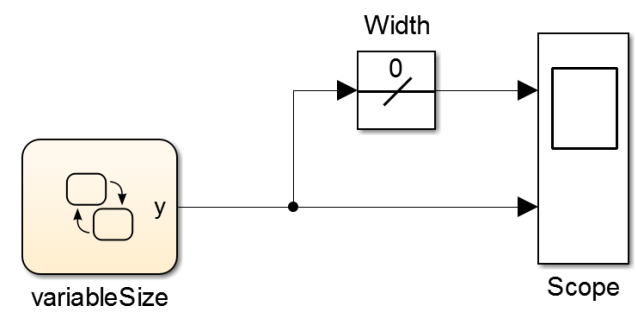

(b) System "sf_variable_size_data" in sf_variable_size_data.mdl.

Figure 6.12: SIMONE can detect system "Length Adaptation" and "sf_variable_size_data" was a clone pair and the similarity of this clone pair was $76 \%$ before contextualization.

The variation in the level of similarity is ranging from $-4 \%$ to $+7 \%$, and most of the changes are within $\pm 2 \%$. Some small size clones like 126 lines clones can get $7 \%$ change, and some larger clones do not change at all. There are 497 clone pairs in category two. The similarity is ranging from $71 \%$ to $89 \%$ with most of them having $71 \%, 72 \%$ and $73 \%$ similarity. Figure 6.12 shows an example of category two, they are a clone pair with $76 \%$ similarity at 118 lines comparison before contextualization. As System "sf_variable_size_data" in Figure 6.12(b) contains a chart, the ten lines change make this clone pairs apart.

Figure 6.13 shows an example of category three, this is a new clone pair with $100 \%$ similarity. Before the contextualization, the lines of these two systems are less than 100 lines, which are not the potential clone candidates. The added weighted lines make them 


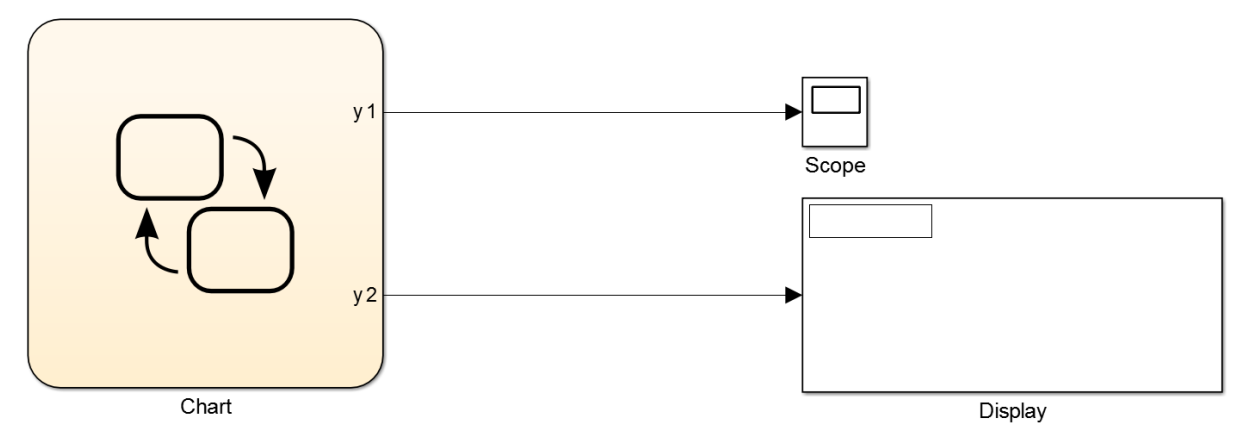

(a) System "sf_resolve_signal_object" in sf_resolve_signal_object.mdl.

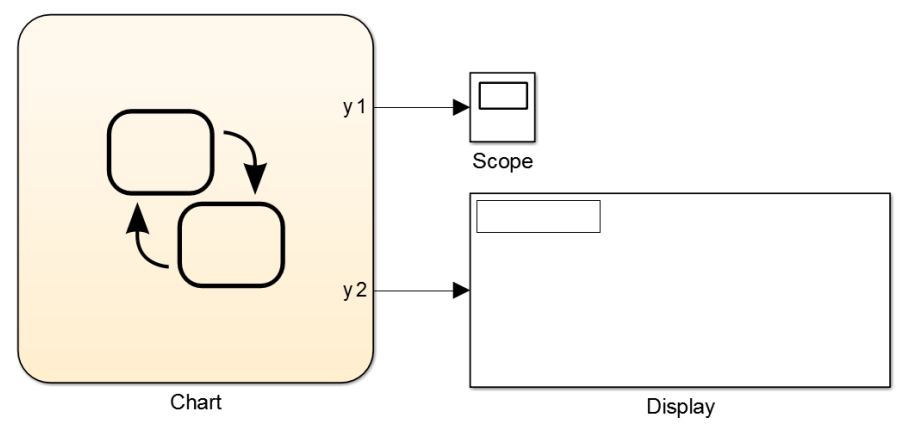

(b) System "doc_resolve_signal_object" in doc_resolve_signal_object.mdl.

Figure 6.13: SIMONE can detect system "sf_resolve_signal_object" and "doc_resolve_ signal_object" was a clone pair and the similarity of this clone pair was $100 \%$ after contextualization.

clone pairs.

\subsubsection{Contextualization discussion}

From the above experiments, we can see each approach has its own merits. Contextualization via Full lines can obtain the best result of the combination clone detection, if the goal of the model clone detection is to identify the duplication of Simulink models that contain identical or similar Stateflow models. The comparison of clone detection will take into account every line of both Simulink and Stateflow models in the full lines approach; and we can examine model clones from a big picture perspective. If the goal of model clone 
detection is more focus on the Simulink models, then the contextualization via one line approach would be better. In this approach, the Stateflow model is just represented as one single line in its parent Simulink model, so it will not affect the comparison of Simulink too much. Meanwhile, we still have the Stateflow information inside the Simulink model. Contextualization via weighted lines presents a more flexible way to detect the duplication of combining two type of models. It can avoid the Stateflow model overwhelm the Simulink model and also remain enough Stateflow model information during the comparison.

\subsection{Summary}

In this chapter, we explained the contextualization phase in more detail. We have briefly discussed the relation between a Simulink block and a Stateflow chart, followed by different approaches to embed the chart into its parent block. We give some examples of each approach and a brief explanation of the contextualized clone detection result. Chapter 7 concludes and outlines possible future work. 


\section{Chapter 7}

\section{Conclusions and Future Work}

This chapter summarizes the work we have done for clone detection in Matlab Stateflow models and provides directions of potential future work to improve our clone detector SIMONE.

\subsection{Summary}

SIMONE has been successfully used in finding near miss subsystem clones in Simulink models [2]. It adapted a text based code clone detector $\mathrm{NiCad}$ to enable the identification of graphical models clone. The adaptation of code clone to models requires several steps. First, a grammar for Simulink .mdl files that parses the textual representation of Simulink models. Second, a specified granularity that identifies the potential candidate similarities at a certain level. In the programing language code clone detection, a certain level of granularity can be functions, blocks, statements, etc. In Simulink model clone detection, the systems level is the appropriate granularity. In addition, SIMONE implemented the corresponding system extractor for Simulink systems to extract the submodels to be compared for similarity. Third, a normalization process is needed to remove the irrelevant elements, rename the value of elements and sort the model elements in the textual form. All of these 
steps are implemented in TXL as the source transformation rules.

In this thesis, we present a solution, which is an extension for SIMONE, to perform clone detection in Stateflow models. We use the Stateflow models in Matlab example data set to experiment with. The solution starts with defining a TXL grammar for Stateflow that parses the models. The textual representation of Stateflow models has a flat structure without a proper granularity to be used as the unit of comparison for clone detection. Thus, it is necessary to transform the presentation to a hierarchical structure and identify the clone fragment as a whole unit. Representation transformation stage not only transforms the sequential representation into a nested version, but also separates the actions of the state from the name of the state, representing each as separate attribute.

We define two levels of granularity charts and states in SIMONE to identify model clones for Stateflow models. Two extractors plugin are implemented in TXL for SIMONE accordingly. Charts in Stateflow represents entire machines. States embed in Charts to present a mode of an event-driven systems. States can contain other Stateflow objects to form a multilevel complex state in a hierarchical structure. The extractors extract either all charts or all states from the Stateflow model files as clone candidates.

Upon analyzing the extracted clone candidates of textual model representation, a set of normalization steps are necessary. The first normalization is filtering, which removes the irrelevant elements and attributes from the potential clones by subjectivity to decide which of the attributes are important and which are irrelevant, such as position, fontSize and zoomFactor are irrelevant elements. The second one is renaming, which gives all the attributes the same value. Renaming significantly improved the similarity, in addition, new cloned pairs are found. The last normalization is sorting, which sorts each Stateflow object by its size in the textual level and the textual elements of each object. The normalization 
process improves the precision and recalls of the clone detention phase.

Besides identifying Stateflow model clones, we also investigate explicating the state machines into the parent Simulink model in a process called contextualization by using similarity of state machines to improve the accuracy of Simulink clones. The contextualization is very similar to the work done by Martin et al. [34]. We import all the state charts referenced by the Simulink blocks into the self-contained unit in the textual representation level by using three different ways full lines, one line, and weighted lines.

\subsection{Future Work}

The capabilities of Simone could be further improved in several research directions. The initial clone detection results from the Matlab example set are similar machines with variations in labels(i.e. state and transition names) and other attributes such as position. We still need to evaluate our approach on more Stateflow models, as well as to refine our SIMONE plugin to improve clone detections. We also found some clone classes that appear to be

embedded Matlab code for use by state and transition labels. Improving our approach to better deal with embedded code is also a line of future research. We also can address our model clone issues further to turn the model clones into model patterns, so that we can better assist and understand model reuse in model development environment. 


\section{Bibliography}

[1] Bakr Al-Batran, Bernhard Schätz, and Benjamin Hummel. Semantic clone detection for model-based development of embedded systems. In Proceedings of the 14th International Conference on Model Driven Engineering Languages and Systems, MODELS'11, pages 258-272, Berlin, Heidelberg, 2011. Springer-Verlag.

[2] M.H. Alalfi, J.R. Cordy, T.R. Dean, M. Stephan, and A. Stevenson. Models are code too: Near-miss clone detection for Simulink models. In ICSM, pages 295-304, 2012.

[3] M.H. Alalfi, J.R. Cordy, T.R. Dean, M. Stephan, and A. Stevenson. Near-miss model clone detection for Simulink models. In IWSC, pages 78-79, 2012.

[4] E.P. Antony, M.H. Alalfi, and J.R. Cordy. An approach to clone detection in behavioural models. In Reverse Engineering (WCRE), 2013 20th Working Conference on, pages 472-476, Oct 2013.

[5] M. Balazinska, E. Merlo, M. Dagenais, B. Lague, and Kostas Kontogiannis. Measuring clone based reengineering opportunities. In Software Metrics Symposium, 1999. Proceedings. Sixth International, pages 292-303, 1999.

[6] Hamid Abdul Basit and Stan Jarzabek. Detecting higher-level similarity patterns in programs. SIGSOFT Softw. Eng. Notes, 30(5):156-165, September 2005. 
[7] S. Bellon, R. Koschke, G. Antoniol, J. Krinke, and E. Merlo. Comparison and evaluation of clone detection tools. Software Engineering, IEEE Transactions on, 33(9):577-591, Sept 2007.

[8] James R. Cordy. The TXL source transformation language. Sci. Comput. Program., 61(3):190-210, 2006.

[9] James R. Cordy. Submodel pattern extraction for simulink models. In Proceedings of the 17th International Software Product Line Conference, SPLC '13, pages 7-10, New York, NY, USA, 2013. ACM.

[10] James R Cordy, Thomas R Dean, Andrew J Malton, and Kevin A Schneider. Source transformation in software engineering using the TXL transformation system. Information and Software Technology, 44(13):827 - 837, 2002. Special Issue on Source Code Analysis and Manipulation (SCAM).

[11] James R. Cordy and Chanchal K. Roy. The NiCad clone detector. In Proceedings of the 2011 IEEE 19th International Conference on Program Comprehension, ICPC '11, pages 219-220, Washington, DC, USA, 2011. IEEE Computer Society.

[12] F. Deissenboeck, M. Pizka, and T. Seifert. Tool support for continuous quality assessment. In Software Technology and Engineering Practice, 2005. 13th IEEE International Workshop on, pages 127-136, 2005.

[13] Florian Deissenboeck, Benjamin Hummel, Elmar Juergens, Michael Pfaehler, and Bernhard Schaetz. Model clone detection in practice. In IWSC, pages 57-64, 2010.

[14] Florian Deissenboeck, Benjamin Hummel, Elmar Jürgens, Bernhard Schätz, Stefan Wagner, Jean-François Girard, and Stefan Teuchert. Clone detection in automotive 
model-based development. In Proceedings of the 30th International Conference on Software Engineering, ICSE '08, pages 603-612, New York, NY, USA, 2008. ACM.

[15] David Harel. Statecharts: A visual formalism for complex systems. Science of Computer Programming, 8(3):231-274, June 1987.

[16] Christian Heinzemann, Jan Rieke, Jana Bröggelwirth, Andrey Pines, and Andreas Volk. Translating mechatronic uml models to matlab/simulink and stateflow. Technical report, Software Engineering Group, Heinz Nixdorf Institute, University of Paderborn, Zukunftsmeile 1, 33102 Paderborn, Germany, March 2014.

[17] Daniel S. Hirschberg. Algorithms for the longest common subsequence problem. $J$. ACM, 24(4):664-675, October 1977.

[18] Benjamin Hummel, Elmar Juergens, and Daniela Steidl. Index-based model clone detection. In Proceedings of the 5th International Workshop on Software Clones, IWSC '11, pages 21-27, New York, NY, USA, 2011. ACM.

[19] The MathWorks Inc. Stateflow Hierarchy of Objects. http : //Www . mathworks . com/help/stateflow/ug/stateflow-hierarchy-of-objects. html, 2014. [Online; accessed March-2014].

[20] The MathWorks Inc. Stateflow user's guide. 2014.

[21] Elmar Juergens, Florian Deissenboeck, and Benjamin Hummel. Clonedetective - a workbench for clone detection research. In Proceedings of the 31st International Conference on Software Engineering, ICSE '09, pages 603-606, Washington, DC, USA, 2009. IEEE Computer Society. 
[22] Elmar Juergens, Florian Deissenboeck, Benjamin Hummel, and Stefan Wagner. Do code clones matter? In Proceedings of the 31st International Conference on Software Engineering, ICSE '09, pages 485-495, Washington, DC, USA, 2009. IEEE Computer Society.

[23] Rainer Koschke. Survey of Research on Software Clones. In Dagstuhl Seminars, 2006.

[24] Hui Liu, Zhiyi Ma, Lu Zhang, and Weizhong Shao. Detecting duplications in sequence diagrams based on suffix trees. In Software Engineering Conference, 2006. APSEC 2006. 13th Asia Pacific, pages 269-276, Dec 2006.

[25] Doug Martin and James R. Cordy. Towards web services tagging by similarity detection. In Mark Chignell, James Cordy, Joanna Ng, and Yelena Yesha, editors, The Smart Internet, volume 6400 of Lecture Notes in Computer Science, pages 216-233. Springer Berlin Heidelberg, 2010.

[26] Douglas Martin and James R. Cordy. Analyzing web service similarity using contextual clones. In Proceedings of the 5th International Workshop on Software Clones, IWSC '11, pages 41-46, New York, NY, USA, 2011. ACM.

[27] Hoan Anh Nguyen, Tung Thanh Nguyen, Nam H. Pham, Jafar M. Al-Kofahi, and Tien N. Nguyen. Accurate and efficient structural characteristic feature extraction for clone detection. In Proceedings of the 12th International Conference on Fundamental Approaches to Software Engineering: Held As Part of the Joint European Conferences on Theory and Practice of Software, ETAPS 2009, FASE '09, pages 440-455, Berlin, Heidelberg, 2009. Springer-Verlag. 
[28] N.H. Pham, H.A. Nguyen, T.T. Nguyen, J.M. Al-Kofahi, and T.N. Nguyen. Complete and accurate clone detection in graph-based models. In Software Engineering, 2009. ICSE 2009. IEEE 31st International Conference on, pages 276-286, May 2009.

[29] Dhavleesh Rattan, Rajesh Kumar Bhatia, and Maninder Singh. Software clone detection: A systematic review. Information \& Software Technology, 55(7):1165-1199, 2013.

[30] Chanchal K. Roy. Detection and Analysis of Near-Miss Software Clones,. Ph.D. dissertation, Queen's University, August 2009. Kingston, ON, Canada.

[31] Chanchal K. Roy, James R. Cordy, and Rainer Koschke. Comparison and evaluation of code clone detection techniques and tools: A qualitative approach. Science of Computer Programming, 74(7):470 - 495, 2009.

[32] Chanchal Kumar Roy and James R. Cordy. A survey on software clone detection research. SCHOOL OF COMPUTING TR 2007-541, QUEEN'S UNIVERSITY, 115, 2007.

[33] C.K. Roy and J.R. Cordy. NiCad: Accurate detection of near-miss intentional clones using flexible pretty-printing and code normalization. In Program Comprehension, 2008. ICPC 2008. The 16th IEEE International Conference on, pages 172-181, June 2008.

[34] J.R. Cordy S. Grant, D. Martin and D. Skillicorn. Contextualized semantic analysis of web services. In WSE 2011, pages 33-42, 2011.

[35] M. Stephan, M.H. Alafi, A. Stevenson, and J.R. Cordy. Towards qualitative comparison of simulink model clone detection approaches. In IWSC, pages 84-85, 2012. 
[36] Andrew Stevenson and James R. Cordy. Grammatical inference in software engineering: an overview of the state of the art. In Hedin (Eds.), Pre-proceedings of the Fifth International Conference on Software Language Engineering (SLE 2012), Fakultät Informatik, Technische Universität, pages 206-225, 2012.

[37] Harald Störrle. VMQL: A generic visual model query language. In Visual Languages and Human-Centric Computing, 2009. VL/HCC 2009. IEEE Symposium on, pages 199-206, Sept 2009.

[38] Harald Störrle. Towards clone detection in uml domain models. Software and Systems Modeling, 12(2):307-329, 2013. 